\title{
Versuche zur Festlegung der Rechenwerte von
} Mauerwerksfestigkeiten

\section{Working Paper}

\section{Author(s):}

Guggisberg, Roland; Thürlimann, Bruno

Publication date:

1987

Permanent link:

https://doi.org/10.3929/ethz-a-000484143

\section{Rights / license:}

In Copyright - Non-Commercial Use Permitted

\section{Originally published in:}

Bericht / Institut für Baustatik und Konstruktion ETH Zürich 7502(5) 
Versuche zur Festlegung der Rechenwerte von Mauerwerksfestigkeiten

Roland Guggisberg

Bruno Thürlimann 
CIP-Titelaufnahme der Deutschen Bibliothek

\section{Guggisberg, Roland:}

Versuche zur Festlegung der Rechenwerte von

Mauerwerksfestigkeiten / von Roland Guggisberg; Bruno

Thürlimann. -Basel; Boston; Berlin: Birkhäuser, 1988

(Bericht / Institut für Baustatik und Konstruktion Zürich; Nr. 7502-5)

ISBN 3- 7643- 1995-X

NE: Thürlimann Bruno:; Institut für Baustatik und Konstruktion

Zürich>: Bericht

WG: 38

DBN 88.037609.0

88.02 .29

0081

cd

Nachdruck verboten.

Alle Rechte, insbesondere das der Übersetzung in fremde Sprachen und der Reproduktion auf photostatischem Wege oder durch

Mikrofilm, vorbehalten. 
Versuche zur Festlegung der Rechenwerte von Mauerwerksfestigkeiten

von

Dipl. Ing. Roland Guggisberg

Prof. Dr. Bruno Thürlimann

Institut für Baustatik und Konstruktion

Eidgenössische Technische Hochschule Zürich 


\section{INHALTSVERZEICHNIS}

Seite

1. EINLEITUNG 1

1.1 Zielsetzung 1

1.2 Problemstellung 2

1.3 Versuchsprogramm 4

2. VERSUCHSKOERPER 6

2.1 Beschreibung 6

2.2 Verwendete Materialien 7

2.2.1 Steine 7

2.2.2 Mörtel 7

3. VERSUCHSDURCHFUEHRUNG 9

3. 1 Versuchsanlage 9

3.1.1 Belastungseinrichtung 9

$\begin{array}{lr}3.1 .2 \text { Messdatenerfassung } & 10\end{array}$

$\begin{array}{ll}3.2 \text { Versuchsablauf } & 10\end{array}$

3.3 Messprogramm 11

3.3.1 Last 11

3.3.2 Verformungsmessungen $\quad 11$

3.3.3 Rissmessungen $\quad 12$

4. VERSUCHSRESULTATE

4. 1 Auswertung 13

4.1.1 Last und Verformungen 13

4.1.2 Rissbilder 14

4.2 Trag-, Riss- und Bruchverhalten 15

4.2.1 Verhalten der Standardserie $\quad 15$

4.2.2 Verhalten der weiteren Versuchsserien 18

$\begin{array}{ll}4.3 \text { Steifigkeiten } & 20\end{array}$

4.4 Rissweiten 21

4.5 Bruchlasten 21

4.6 Isomodu1-Verbandmauerwerk 22

4.7 Streuung der Versuchsresultate 22

5. VORSCHLAG FUER RECHENWERTE DER MAUERWERKSFESTIGKEIT 23

5.1 Bruchbedingung für Mauerwerk 23

5.2 Parameterwerte aus den Versuchsresultaten 25

5.3 Unsicherheiten bei der Bestimmung der Parameter 26

$\begin{array}{ll}5.4 \text { Mauerwerks- und Steinfestigkeiten } & 27\end{array}$ 
6. ABSCHLIESSENDE BETRACHTUNGEN 29

6.1 Qualitative Beschreibung des Tragverhaltens 29

6.2 Anregungen zu Verbesserungen 31

6.3 Vorschläge für das weitere Vorgehen 32

ZUSAMMENFASSUNG

RESUME $\quad 36$

SUMMARY

VERDANKUNGEN $\quad 40$

LITERATURVERZEICHNIS

BEZEICHNUNGEN

TABELLEN $1-8 \quad 44$

$\begin{array}{lll}\text { BILDER } 1-28 & 48\end{array}$

ANHANG A: Tabellen mit Versuchsresultaten $\quad 79$

ANHANG B: Zusatzversuche $\quad 88$

ANHANG C: Provenienzen der Mauersteine 95

VERSUCHSPROGRAMM (aufklappbare Seite) 


\section{EINLEITUNG}

\section{$1.1 \quad$ Zielsetzung}

Horizontal auf ein Bauwerk wirkende Kräfte, zum Beispiel aus Wind oder Erdbeben, müssen durch schubsteife Tragwerksteile wie Treppenhauskerne oder tragende Wände in die Fundamente abgeleitet werden. Wohn- oder Bürogebäude mit vier bis sechs Geschossen werden meistens nicht mit einem tragenden Stahlbetonskelett erstelit, sondern die Wände werden in Mauerwerk und lediglich die Decken in Stahlbeton ausgeführt. Dies führt zu einem bedeutenden Anteil des Mauerwerks am Baumarkt. Bis vor wenigen Jahren waren die Tragfähigkeit und das Tragverhalten von Mauerwerk wissenschaftlich kaum erforscht.

Um eine wirtschaftlichere Bauweise erzielen zu können wäre es interessant, dem Mauerwerk komplexere Tragfunktionen zuzuordnen. Allgemein sind die Anforderungen an die Gebrauchstauglichkeit, die Sicherheit, die Dauerhaftigkeit und den Nutzwert der Bauwerke gestiegen und deshalb sind international Bestrebungen im Gange, Grundlagen für eine wirtschaftlichere und sicherere Bemessung von Mauerwerk zu schaffen.

Verschiedene Forschungsprojekte des Institutes für Baustatik und Konstruktion der ETH Zürich befassen sich mit der Tragfähigkeit von Mauerwerk [1-8]. In einer Forschungsarbeit wurde die Schubtragfähigkeit geschosshoher Mauerwerkswände untersucht [4]. Das Ziel dieser Arbeit war die Entwicklung eines Verfahrens zur Bemessung von Mauerwerksscheiben unter Normallast und Schub. Als Grundlage wurden vorgängig Untersuchungen über die Festigkeit von Mauerwerk unter ebenen zweiachsigen Spannungszuständen vorgenommen [1]. Aus den Erkenntnissen dieser Versuche wurde eine Bruchbedingung für Mauerwerk hergeleitet [3]. Diese Bruchbedingung und das Bemessungsverfahren wurden in einer Dissertation umfassend dargestellt [2].

Da die Vielfalt der Mauerwerksprodukte sowohl in der Schweiz als auch im Ausland sehr gross ist, wurden verschiedene materialbedingte Einflussgrössen auf die Bruchbedingung für Mauerwerk in einem Anschlussprogramm untersucht und im vorliegenden Bericht beschrieben. Die Zielsetzung war, im Hinblick auf eine bevor 
stehende Neufassung der Schweizer Norm SIA 177 "Mauerwerk" [10] durch Versuche Rechenwerte von Festigkeiten für Backstein- und Kalksandstein-Mauerwerk zu ermitteln. In den Versuchen wurden Steine verschiedener Provenienz und verschiedene Mörtelmischungen verwendet. Der Auswahl lag der Wunsch zugrunde, eine repräsentative Uebersicht über die Unterschiede in den Mauerwerksfestigkeiten zu gewinnen. Hinzu kam das Ziel, einzelne Einflussgrössen möglichst isoliert zu untersuchen. Die Auswahlkriterien werden im Abschnitt 1.2 erläutert.

Das gemeinsame Ziel aller Teilprojekte ist, eine wissenschaftlich fundierte Basis für eine wirtschaftlichere und sicherere Bemessung von Mauerwerkswänden zu schaffen und Ansatzpunkte für künftige Verbesserungen des Baustoffes Mauerwerk aufzuzeigen.

\subsection{Problemstellung}

Das Mauerwerk ist ein inhomogenes, anisotropes Material. Es ist aus den zwei Komponenten Stein und Mörtel aufgebaut. Bedingt durch die Herstellung (z.B. Extrusion bei Backsteinen) kann bereits das Grundmaterial der Steine eine Richtungsabhängigkeit der Festigkeitseigenschaften aufweisen. Die Steine in der Schweiz weisen meistens eine Lochung in vertikaler Richtung auf. Somit ist die Nettofläche eines Horizontalschnittes wesentlich grösser als eines Vertikalschnittes. Die Mörtelfugen wirken als orientierte Schwächungszonen.

Die Tragfähigkeit des Mauerwerks hängt somit - einfach ausgedrückt - von der Richtung $a b$, mit welcher eine Kraft in der Wandscheibe abgetragen wird. Im allgemeinen treten bei Scheiben zweiachsige Spannungszustände auf. Die Bruchbedingung gibt an, wie gross die Spannungen werden dürfen, bis das Mauerwerk versagt; die experimentelle Absicherung muss im allgemeinen mit zweiachsiger Beanspruchung erfolgen [1].

Die Zielsetzung dieser Forschungsarbeit galt hingegen nicht einer Ueberprüfung der Bruchbedingung, sondern der Bestimmung der Parameter derselben mit Steinen verschiedener Provenienzen und mit verschiedenen Mörtelmischungen. Daher wurden zur Verringerung des Aufwandes einachsige Versuche gewählt. 
Es wurden verschiedene Einflussgrössen auf die Parameter der Bruchbedingung untersucht: Grundmaterial, Lochbild, Lochanteil und Format der Steine, verschiedene Mörtelmischungen und Ausbildungen der Stossfugen. Die Tabelle 1 zeigt eine Uebersicht der Parameter des Versuchsprogrammes. Sie wird durch folgende Erläuterungen ergänzt:

- In der Versuchsserie 2 wurde ein Stein verwendet, der dem Stein der Versuche K1 bis $\mathrm{K} 12$ der Versuchsserie 1 (Versuche über die Festigkeit von zweiachsig beanspruchtem Mauerwerk [1]) gut entsprach. Von diesem Stein (Provenienz B) wurde eine ausreichende Menge aus der gleichen Produktionscharge für Versuchszwecke an Lager gelegt. Mit dem gleichen Stein wurden bereits die Versuche an geschosshohen Schubwänden [4] unternommen. Deshalb wird die Serie 2 als Standardserie bezeichnet.

- Mit der Serie 3 wurde abgeklärt, inwiefern sich eine versetzte Anordnung der Löcher im Stein auf die Tragfähigkeit auswirkt. Der Stein der Serie 3 ist ansonsten gleichwertig mit dem Stein der Serie 2. Dieser Stein weist aber parallel zur Wirkungsebene der resultierenden Kraft keine durchgehenden Stege auf, die sich direkt auf die Vermörtelung der Stossfugen abstützen können. Weitere Erläuterungen dazu enthält Kapitel 6.

- In den Serien 4, 5 und 6 wurden Steine mit Normalformat verwendet. Für die Serien 4 und 5 wurde ein Stein mit niedriger Festigkeit gesucht. Der Stein von Serie 4 wurde in der Serie 5 mit verlängertem Mörte1, d.h. mit einem Mörtel. bei dem ein Teil des Zementes durch Kalk ersetzt wurde, und der somit eine geringere Festigkeit aufweist, vermauert. Solche verlängerte Mörtel werden wegen der besseren Verarbeitbarkeit noch häufig verwendet.

- Für die Serie 6 wurde ein Stein mit möglichst hoher Festigkeit ausgesucht. Nach der Normprüfung wurde dieser Stein qualitativ ungefähr gleich beurteilt wie der Stein der Serie 2.

- Es erschien interessant, einen Stein mit möglichst geringem Lochanteil zu suchen. In der Schweiz werden Vollsteine aber kaum verwendet. Deshalb wurde für die Serie 7 ein Schalldämmstein gewählt. Trennwände aus Schalldämmsteinen werden oft ausgeführt. Wände aus solchen Steinen sollten auch zur Abtra- 
gung von Schubkräften günstig sein. Deshalb schien diese Wahl in zweifacher Hinsicht interessant. Der Schalldämmstein CALMO ist ein halbhoher Modulstein, und demzufolge unterschied sich das Steinformat bei der Versuchsserie 7 wie bei der Serie 8 von demjenigen der anderen Serien.

- Zur Abklärung des Einflusses des Steinformates auf das Tragverhalten wurde ein mit dem Stein B der Serie 2 vergleichbarer Sichtstein mit halbhohem Modulformat ausgesucht.

- Mit der Versuchsserie 9 wurde ein Verbandmauerwerk mit integrierter Isolation geprüft. Das hier verwendete Mauerwerk mit der Bezeichnung ISOMODUL SUPER ist aus einem Spezialstein und einem Ausgleichsstein aufgebaut.

- Die Versuchskörper der Serie 10 waren im Werk vorgefertigte Wände mit der Bezeichnung PRE TON. Bei diesem vorgefertigten Mauerwerk wurde ein Gussmörtel mit spezieller Zusammensetzung verwendet.

- Die Versuchsserien 11 und 12 wurden mit Kalksandstein-Mauerwerk durchgeführt, wobei der Stein der Serie 12 teilweise nicht durchgehende Löcher aufwies, d.h.. der Stein wies in der einen Lagerfugenfläche weniger Löcher auf als in der anderen. Zur Untersuchung des Einflusses der Makroverzahnung der Mörtelzapfen in den Löchern des Steins erschien dieser Unterschied aufschlussreich.

\subsection{Versuchsprogramm}

Die hier beschriebenen 11 Versuchsserien wurden mit Mauerwerk aus 11 verschiedenen Steinen, kombiniert mit drei verschiedenen Mörtelmischungen, durchgeführt. Das Versuchsprogramm ist in der Tabelle 2 zusammengeste $11 t$. Die früher durchgeführte Versuchsserie 1 umfasst die Versuche K1 bis K12 [1]. Pro Serie wurden Versuchskörper mit bis zu fünf verschiedenen Fugenneigungswinkeln $\propto\left(0^{\circ}, 22.5^{\circ}\right.$, $45^{\circ}, 67.5^{\circ}$ und $90^{\circ}$ ) geprüft. In der Versuchsserie 2 (Standardserie) wurden Vorversuche mit den Winkeln $\alpha=22.5^{\circ}$ und $\alpha=45^{\circ}$ durchgeführt, um Aufschluss über die Streuung der Resultate gleicher Versuche zu erhalten. 
Bei den meisten Versuchsserien wurden Zusatzversuche R1 bis R15 (R steht für RILEM) mit Probekörpern nach der RILEM-Richtlinie durchgeführt; sie sind im Anhang B aufgeführt und erklärt. Am Schluss des Versuchsberichtes befindet sich zwecks besserer Uebersicht über die Versuche und die Bezeichnungen eine aufklappbare Seite mit dem Versuchsprogramm. 


\section{VERSUCHSKOERPER}

\subsection{Beschreibung}

Die Versuchskörper massen $1200 \times 1200 \mathrm{~mm}$ und waren mit Ausnahme derjenigen der Serie 9 (Verbandmauerwerk) als Einsteinmauerwerk im Läuferverband ausgeführt. Die Wandstärke richtete sich nach dem in der Baupraxis am häufigsten ausgeführten Wert von $150 \mathrm{~mm}$. Die Abmessungen der Versuchskörper mit $90^{\circ}$ Fugenneigung entsprachen ungefähr denjenigen der RILEM-Körper ( $s$. Anhang B). Aus den Vorversuchen ergab sich für diese Körper, dass die Anzahl Stossfugen unbedingt in jeder Steinlage gleich sein muss (Bilder 1, 2, 3).

Als Versuchsserie 9 wurde ein Verbandmauerwerk, aufgebaut aus dem Spezialstein ISOMODUL SUPER und einem $75 \mathrm{~mm}$ starken Ausgleichsstein mit eingefügter $50 \mathrm{~mm}$ starker Wärmedämmung geprüft. Die Wandstärke betrug $325 \mathrm{~mm}$ (Bild 5). Die Läuferschicht des Spezialsteins war zur Läuferschicht des Ausgleichssteins um eine halbe Steinlänge versetzt. Die Lagerfuge war im Bereich der Wärmedämmung nicht vermörte1t; die Stossfuge war ohne Mörtel ausgeführt.

Die Versuchskörper wurden in der Versuchshalle des Prüf- und Forschungsinstitutes des Verbandes der Schweizerischen Ziegel- und Steinfabrikanten (VSZS) in Sursee hergestellt und geprüft. Sie wurden von den Handwerkern des Institutes nach der geltenden Baupraxis aufgemauert. Der Mörtel der Lagerfuge wurde in üblicher handwerklicher Manier ausgelegt. Die Steine wurden mit einem Gummihammer leicht angeklopft, so dass die Lagerfugen stets voll verfüllt waren. Die Lagerfugen wiesen beim Mauerwerk aus Modulsteinen ein Stärke von $10 \mathrm{~mm}$ und beim Mauerwerk aus Normalformatsteinen eine solche von $15 \mathrm{~mm}$ auf. Die Stossfugen wurden entsprechend den vom Zieglerverband herausgegebenen Empfehlungen ausgeführt (Bild 4), d.h. beim Modulstein als Pressfuge mit verdecktem Spatz, beim Normalstein mit einem auf jede Kante der Stossfugenfläche aufgetragenen Spatz und beim Sichtstein und beim Schalldämmstein CALMO mit voll vermörtelten Stossfugen. Die Stossfugen, wenn nicht als Pressfugen ausgeführt, waren $10 \mathrm{~mm}$ stark.

Die Maurer des Prüfinstitutes arbeiteten sehr exakt. Die Versuchswände waren sowohl vertikal als auch horizontal gut eingefluchtet. Die Lagerfugen waren gerade und über die ganze Länge gleich stark, was die ausführungsbedingte Streuung der Versuchsresultate auf ein Minimum reduzierte. 
Die Versuchskörper mit geneigten Fugen wurden zwischen zwei senkrecht stehenden Stahlprofilen mit einer Spanplatte als Rückwand aufgemauert. Die Randsteine wurden vorgängig mit einer Säge entsprechend zugeschnitten. Die Versuchswände mit einer Fugenneigung von $67.5^{\circ}$ wurden als Wände mit $22.5^{\circ}$ Neigung erstellt und nach dem Erhärten des Mörtels beim Einbau in die Prüfmaschine vorsichtig um $90^{\circ}$ gekippt. Entsprechend wurden auch die $90^{\circ}$-Körper hergestel1t.

Die Versuchskörper aus vorfabriziertem Mauerwerk wurden aus grösseren Tafeln herausgeschnitten. Die Lagerfugen wurden maschinell mit einem sehr plastischen Mörtel gegossen. Die Handlöcher und die Vertiefungen in den Stossfugenflächen der Steine wurden dabei mit Mörtel verfüllt. Die Ausführung der Stossfugen entsprach demzufolge derjenigen beim Mauerwerk aus Modulsteinen.

\subsection{Verwendete Materialien}

\subsubsection{Steine}

In der Tabelle 3 sind die Versuchsreihen mit den jeweils verwendeten Steintypen aufgeführt. Die Provenienz der Steine (Spalte 2) ist mit einem Buchstaben bezeichnet, dessen Bedeutung im Anhang $C$ aufgeschliusselt ist. In der Spalte 3 sind die Steine nach dem in der Schweiz gebräuchlichen Code bezeichnet. Der Steinquerschnitt (Spalte 4) zeigt anschaulich das Lochbild der Steine. Im weiteren sind die Resultate der nach der Schweizer Norm SIA 177 [10] durchgeführten Prüfungen dargestellt. Die Druckprüfung wird an plangeschliffenen Steinen durchgeführt. Für die Bestimmung der Querzugfestigkeit werden drei plangeschliffene Steine mit Zwischenlagen aus einem definierten Elastomer aufeinandergestellt und vertikal gedrückt. Die Festigkeitswerte sind auf den Bruttoquerschnitt bezogen. Bezüglich weiterer Angaben gibt die Norm SIA 177 Auskunft.

\subsubsection{Mörtel}

Es wurden drei verschiedene Mörtelmischungen verwendet:

- Zementmörtel mit $350 \mathrm{~kg}$ Zement/m³ fertige Mischung,

- verlängerter Mörtel (100 kg Zement und $250 \mathrm{~kg}$ hydraulischer Kalk) sowie

- Gussmörtel für die vorfabrizierten Wände. 
Die beiden ersteren wurden nach Rezepten des Prüf- und Forschungsinstitutes hergestellt. Der Sand wies eine stetige Siebkurve, einen leichten Ueberschuss an Siltfraktion und ein Grösstkorn von $3 \mathrm{~mm}$ auf. Für den Gussmörtel wurde dem Sand mit $2 \mathrm{~mm}$ Grösstkorn noch Filler zugegeben. Das Mischverhältnis Sand/Zement/Filler betrug 3:1:1, der Wasser/Zement-Faktor betrug 1.0. Um die Verarbeitbarkeit zusätzlich zu verbessern, wurde der Mischung ein handelsüblicher Verflüssiger beigefügt.

Von jeder Mörtelmischung wurden sechs Prismen von $40 \times 40 \times 160 \mathrm{~mm}$ hergestellt und einer Prüfung nach Norm SIA 177 unterzogen. Die Normprüfung umfasst eine Biegezugprüfung und eine Druckprüfung an den Hälften des Biegezugkörpers. Die Mittelwerte jeder Versuchsserie sind in der Tabelle 4 aufgeführt. 


\section{VERSUCHSDURCHFUEHRUNG}

\subsection{Versuchsanlage}

\subsubsection{Belastungseinrichtung}

Für die Versuchsdurchführung stand eine Tinius 01 sen $8000 \mathrm{kN}-P$ resse, d.h. eine steife Belastungseinrichtung mit moderner elektronischer Steuerung, zur Verfügung.

Für die Einleitung der Kraft in den Probekörper wurde die Einrichtung der früher durchgeführten Versuche [1] übernommen. Die Wahl gründete auf folgenden Ueberlegungen:

- Die Verformung des Probekörpers soll nicht behindert werden, da sich sonst Zwängungsbeanspruchungen aufbauen.

- Bei der gegebenen Problemstellung muss die Kraft gleichmässig über den Querschnitt des Probekörpers verteilt sein.

Dem oberen und dem unteren Rand entlang waren je acht Krafteinleitungselemente verteilt, wobei jedes Element aus einer Elastomerplatte und einer Stahlplatte für die Aufnahme der Querzugkräfte bestand. Bei den Elastomerplatten waren drei Stärken verfügbar. Bei Versuchen, die das Auftreten grösserer Verformungen erwarten liessen, wurden stärkere Platten eingesetzt. Wegen der Ausquetschung des Elastomers musste die verwendete Plattenstärke auf die zu erwartende Höchstlast abgestimmt werden.

Bis zum Erreichen der Höchstlast erfüllten die Krafteinleitungselemente ihre Aufgabe befriedigend. Bei starker Zunahme der Verformung des Probekörpers traten Querdehnungsbehinderungen auf. Im weiteren ist zu bemerken, dass die Elastomerplatten die Steifigkeit der Belastungseinrichtung wesentlich reduzierten, weshalb bei den kleinen Versuchskörpern auf sie verzichtet wurde. 


\subsubsection{Messdatenerfassung}

Bild 6 zeigt die Anordnung der Messdatenerfassung am Versuchskörper und an den Krafteinleitungselementen. Die Verschiebungen auf der Wandoberfläche wurden mit zwei Deformetern gemessen. Dies sind Setzgeräte mit einer festen und einer beweglichen Metallspitze, welche in Vertiefungen der Messbolzen eingeführt werden. Der Deformeter misst mit einem eingebauten induktiven Weggeber die Distanz zwischen zwei Messbolzen auf $\pm 0.003 \mathrm{~mm}$ genau.

Mit einem weiteren Setzgerät, basierend auf einer Messuhr mit $0.002 \mathrm{~mm}$ Ablesegenauigkeit, wurde an vier Stellen der Wand die Veränderung der Wandstärke beobachtet. Dieses mechanische Messgerät erreichte aber die geforderte Messgenauigkeit von $0.01 \mathrm{~mm}$ nicht. Mit vier Messuhren für die vertikale Richtung und mit vier Glaslinealen für die Messung der Schiebungen wurde die Verformung zweier Krafteinleitungselemente am oberen Wandende erfasst. Im weiteren waren vier induktive Weggeber paarweise auf jeder Wandseite angebracht. Sie dienten der Versuchssteuerung und erfassten die Längenänderung des Versuchskörpers in Kraftrichtung auf einer Messlänge von einem Meter.

\subsection{Versuchsablauf}

Nach der Herstellung wurden die Versuchskörper vier Wochen lang in der Versuchshalle gelagert und in dieser Zeit mit den Messbolzen versehen. Für die Messungen der Wandstärken wurden Löcher gebohrt. Anschliessend wurden die Versuchskörper mit weisser Dispersionsfarbe angestrichen, damit beim Versuch die Risse besser sichtbar wurden. Im Alter von 27 bis 37 Tagen wurden die Versuchskörper am Vortag des Versuchs in die Prüfmaschine eingebaut und die Messaparatur installiert.

Die Versuche wurden verformungsgesteuert durchgeführt, d.h. für die Steuerung der Belastungseinrichtung wurde die Verformung der Probe kontrolliert. Die Verformung konnte nicht kontinuierlich bis zum Bruch gesteigert werden, weil während der Deformetermessungen die Verformung konstant gehalten werden muss. Diese Unterbrüche im Belastungsablauf wurden als Laststufen bezeichnet. Sie mussten so verteilt werden, dass die wesentiichen Merkmale des Last-Verformungs-Verhaltens durch die Messungen erfasst werden konnten. Bis zum Erreichen der Höchstlast waren mindestens vier Laststufen vorgesehen. Bei stärkerer Veränderung des Ver 
formungsverhaltens wurden entsprechend mehr Laststufen durchgeführt. Zu Beginn des Versuches war es jeweils schwierig, bis zur nächsten Laststufe Schritte für den Verformungszuwachs vorzugeben, weshalb zusätzlich auch Schritte für den Lastzuwachs formuliert wurden.

Beim Erreichen des gewünschten Ziels betreffend Verformungszuwachs wurde bis zum Beginn der Messungen eine Pause von zwei Minuten eingelegt. Dann wurden die Messwerte für die Last und der Weggeber abgefragt. Mit dem Deformeter wurde zuerst die Verformung des ganzen Versuchskörpers, dann die Verformung von vier Steinen gemessen. Anschliessend wurde die Messung der Last und der Weggeber wiederholt. Während der Deformetermessungen fiel die Last infolge Relaxation des Mauerwerkkörpers vom Anfangswert $F_{A}$ auf den Endwert $F_{E}$. Mit einem Filzschreiber wurden die Risse nachgezeichnet und an einigen Stellen gemessen, die Rissprotokolle nachgeführt und Fotos gemacht. Der Zeitbedarf für eine Laststufe betrug je nach Messaufwand ca. 15 bis 30 Minuten.

\subsection{Messprogramm}

\subsection{Last}

Auf der Anzeige der Prüfmaschine konnte die Last während des Versuchs ständig beobachtet werden. Mit Hilfe eines Schleppzeigers konnten Spitzenwerte festgehalten und jeweils im Versuchsprotokoll vermerkt werden. Ein Schreiber registrierte ca. alle 10 Sekunden die Werte der Last und des Kolbenweges. Für jede Laststufe wurden der Anfangs- und der Endwert der Last erfasst.

\subsubsection{Verformungsmessungen}

Mit induktiven Weggebern wurde die vertikale Verformung des Versuchskörpers gemessen. Diese Messung diente als Information für die Versuchssteuerung und ist demzufolge im Bericht nicht aufgeführt.

Die Verformungsmessung am Versuchskörper erfolgte mit einem Messnetz auf beiden Wandseiten, das nach Möglichkeit die ganze Wandoberfläche (Bild 6) erfasste. Die auf die Mitte der Steine geklebten Messbolzen entsprachen den Knoten des Mess- 
netzes. Somit bestand das Messnetz aus lauter gleichschenkligen Dreiecken mit der Basis gleich einer Steinlänge plus Stossfugenstärke und der Höhe eines Steins plus Lagerfuge.

Für die Messung der Verformungen von Steinen diente ein Messviereck, bestehend aus vier in einem Rechteck angeordneten Messbolzen. Alle Kanten und die beiden Diagonalen wurden gemessen. Es wurde die Verformung von vier Steinen auf beiden Wandseiten erfasst.

\subsubsection{Rissmessungen}

Die von Auge sichtbaren Risse der Wand wurden mit einem Filzschreiber sorgfältig nachgezeichnet, um deren Verlauf besser sichtbar zu machen. An ungefähr zehn markierten Stellen wurden mit einem Mikroskop die Rissweiten auf $0.01 \mathrm{~mm}$ genau gemessen und dann Rissbild und Rissweiten protokolliert. Nach jeder Laststufe wurde der Versuchskörper fotografiert. 


\section{VERSUCHSRESULTATE}

\subsection{Auswertung}

\subsubsection{Last und Verformungen}

Die Messdaten wurden mit dem Computer ausgewertet. Die Messwerte der elektrischen Geräte waren als Stromspannung in Millivolt vorhanden und mussten mit Proportionalitätsfaktoren in die gewünschten Einheiten umgerechnet werden. Die Deformetermesswerte mussten umfangreich weiterbearbeitet werden.

Der Deformeter hat keine integrierte Eichmöglichkeit und reagiert empfindlich auf Temperaturänderungen. In Abständen von ca. zehn Messungen wurden deshalb Eichmessungen bezüglich eines Stabes aus Invarmaterial durchgeführt. Die Messwerte wurden bei der Auswertung mit diesen Eichmessungen linear abgeglichen.

Wie beschrieben, wurden mit dem Deformeter Messnetze ausgemessen. Es interessierten somit nicht die Längenänderungen einzelner Messungen, sondern die Verschiebungen der Messnetzknoten, resp. der Messbolzen bezüglich der unverschobenen Nullage. Dazu wurden die Daten einem Fehlerausgleichsprogramm eingegeben.

In einem weiteren Auswertungsschritt erfolgte die Berechnung von Verzerrungen. Für kleine Verzerrungen gelten folgende kinematischen Beziehungen:

$$
\epsilon_{x}=\frac{\partial}{\partial x} \cdot u(x, y), \quad \epsilon_{y}=\frac{\partial}{\partial y} \cdot v(x, y), \quad \gamma_{x y}=\frac{\partial}{\partial y} \cdot u(x, y)+\frac{\partial}{\partial x} \cdot v(x, y)
$$

Aus den Verschiebungen $u$ und $v$ von jeweils drei Messnetzknoten wurden für den Bereich innerhalb der Knoten die Verzerrungen gerechnet. Im Bild 8 ist die Berechnung der Verzerrungen mit verallgemeinerten Formeln und einer Skizze dargestellt. Die Formeln ergeben sich aus den obigen kinematischen Beziehungen und der Annahme, dass die Verschiebungen innerhalb des dreieckförmigen Bereichs linear verlaufen.

Die Messwerte der Steine wurden in gleicher Weise bearbeitet. Entsprechend der Anordnung der Messbolzen (Bild 6) wurden aus vier Kombinationen zu je drei Messknoten die Verzerrungen gerechnet und dann die Werte gemittelt. 
Für die grafische Darstellung in Last-Verzerrungs-Diagrammen wurden die Verzerrungen eines Teilbereichs gemittelt. Störungen der Verformung im Randbereich des Versuchskörpers blieben somit weitgehend ohne Auswirkung. Die Verzerrungen der vier gemessenen Steine wurden für die Darstellung gemittelt. Da die Verformungsmessungen an Steinen einen viel geringeren Bereich der Wandoberfläche abdeckten als der zur Darstellung verwendete Teil des Messnetzes für die Wandverformungen, können bei den dargestellten Steinverzerrungen lokale Einflüsse eine grössere Auswirkung haben.

Im Bild 9 wurde am Beispiel des Versuchs $K 63$ versucht, den Einfluss lokaler Vorkommnisse wie z.B. der Einfluss von Rissen auf die dargestellten mittleren Verzerrungen zu zeigen. Nebeneinander wurden auf einer Doppelseite für drei Laststufen das Verschiebungsbild des Messnetzes, die Hauptdehnungen (gerechnet für die einzelnen Dreiecke des Messnetzes) und gezeichnete Rissbilder dargestellt. In der Spalte 4 wurden zwei vereinfachte Last-Verzerrungs-Diagramme dargestellt. Für die beiden Diagramme wurden unterschiedliche Bereiche des Messnetzes (punktierte Flächen) für die Mittelung der Verzerrungen berücksichtigt. Das obere Diagramm entspricht demjenigen von Bild 13a. Für das untere Diagramm wurde das Messnetz im Bereich eines klaffenden Risses ausgewertet.

Beim Versuch K 63 war die Darstellung des Verschiebungsbildes besonders aussagekräftig. Die Verschiebungen beziehen sich auf ein Zentrum, für welches willkürlich ein Messnetzknoten in einem Wandbereich mit verschwindend kleinen Verformungen gewählt wurde. Der in der Laststufe 14 erzeugte treppenförmig verlaufende Riss führte nur in $x-R i c h t u n g ~ z u$ Verschiebungen.

\subsubsection{Rissbilder}

Das Rissbild wurde in jeder Laststufe, bei welcher wesentliche Veränderungen vorkamen, fotografiert. Stärkere Risse sind auf den Fotos gut zu erkennen, feinere Risse hätte man ohne Nachzeichnen des Rissverlaufs mit Filzschreiber im Foto kaum erkennen können; die Filzschreiberstriche sollen jedoch keinen falschen Eindruck in bezug auf die Rissöffnung erwecken. Die ungerissenen Mörtelfugen sind auf den Fotos nur schwer zu erkennen. Für eine Interpretation der Rissbilder ist aber der Verlauf der Fugen eine hilfreiche Information. Des 
halb wurden von den Fotos gezeichnete Rissbilder hergestellt (Bilder 15 bis 19); darin sind die Fugen gestrichelt enthalten. Der Verlauf und die Länge der Risse wurden wirklichkeitsgetreu gezeichnet. Die Bilder 20 und 21 enthalten mittlere und maximale Rissweiten.

\subsection{Trag-, Riss- und Bruchverhalten}

Wegen der Vielzahl von Versuchen kann nicht jeder einzeln dargestellt werden. Im Anhang $A$ sind jedoch die gemessenen Lasten und die Mittelwerte der Verzerrungen jedes Versuchs in Tabellen festgehalten. Um die Darstellung der Versuchsresultate übersichtlicher zu gestalten, wurde die Standardserie 2 ausführlicher beschrieben. Für die weiteren Versuchsserien wurden hauptsächlich die Abweichungen vom Verhalten in der Standardserie 2 festgehalten. Alle Last-Verzerrungs-Diagramme sind in den Bildern 10 bis 14 wiedergegeben, wobei jeweils die Versuche mit gleicher Fugenneigung in einem Bild zusammengefasst sind. Der Massstab der Verzerrungsachse ist in allen Diagrammen identisch, derjenige der Lastachse ist für die Diagramme der Versuche mit gleicher Fugenneigung jeweils gleich.

Es ist noch zu bemerken, dass in allen Last-Verzerrungs-Diagrammen der $0^{\circ}-$ und der $90^{\circ}$-Versuche zwecks besserer Lesbarkeit der Diagramme auf die Darstellung der Schiebungen verzichtet wurde. Die Wiedergabe der Schiebungen dieser Versuche hat keinen wesentlichen Informationsgehalt.

\subsubsection{Verhalten der Standardserie}

Die Last-Verzerrungs-Diagramme der Standardserie sind in den Bildern 10 bis 14 jeweils mit a) bezeichnet. Die Fotos des Bruchzustandes sind im Bild 22 zusammengestellt. Das Bild 15 zeigt Zeichnungen typischer Rissbilder der Standardversuche.

\section{$0^{0}$ Fugenneigung:}

Die Stauchungen in x-Richtung nahmen bei steigender Last bis nahe zur Bruchlast praktisch linear zu (Bild 10a). Rund 30\% der Verformungen entfielen auf die Lagerfugen und rund $70 \%$ auf die Steinlagen. Die Dehnung in $y$-Richtung zeigte ab Beginn der Belastung ein positives Vorzeichen. Dabei entsprach die Verformung 
der Wand der Verformung der Steine, was bedeutet, dass die Steine bei vertikaler Belastung der Mauerwerkswand eine Zugdehnung erfahren. Bei ungefähr $75 \%$ der Bruchlast zeigten sich erste Risse. Sie verliefen parallel zur Kraftrichtung, d.h. senkrecht zu den Lagerfugen (Bild 15). Es wurden nur Risse in Steinen beobachtet, da die Stossfugen als Pressfugen ausgeführt waren (vgl. Kapitel 2 und 3). Risse, die in der Flucht von Stossfugen verliefen, waren deutlich in der Minderzahl. Die Risse schienen mehrheitlich von Lagerfugen auszugehen, doch gab es auch Risse, die mitten in Steinen entstanden, ohne dass sie anfänglich die Lagerfugen erreichten. Die Risse konzentrierten sich in einem Band ungefähr in Wandmitte.

Der Bruch erfolgte plötzlich mit einem lauten Knal1. Dabei wurde die Wand fast in der ganzen Höhe parallel zur Wandoberfläche gespalten (Bild 22). Die eine untere Ecke der Wand wurde vollständig zerstört, die äussere Schale der Steine wurde dort abgesprengt, und die Steine selbst wurden in prismenförmige Stücke zersplittert. Die Last fiel augenblicklich auf ungefähr $10 \%$ ab.

\section{$22.5^{\circ}$ Fugenneigung:}

Auch bei diesem Fugenneigungswinkel nahm die Stauchung in $x-R i c h t u n g$ bei steigender Last linear bis zum Bruch der Wand zu (Bild 11a). Die Steifigkeit der Wand in $x$-Richtung war aber geringer als beim $0^{\circ}$-Versuch, und der Anteil der Verformung der Steine an der Verformung der Wand war erstaunlicherweise verschwindend klein. Ebenso überraschend ist, dass keine Dehnungen in y-Richtung feststellbar waren. Die Schiebungen der Wand verliefen bei steigender Last bis zum Bruch linear. Ungefähr 60\% der Schiebungen wurden vom Stein erbracht. Bei $80 \%$ der Bruchlast traten Risse auf. Sie folgten teilweise den Fugen, teilweise verliefen sie durch Steine hindurch, entweder parallel zur Kraftrichtung oder parallel zur $x$-Richtung (Bild 15). Die Risse änderten auch mitten im Stein die Richtung. Beim Versuch K15 waren die Risse auf das eine Wandende konzentriert, beim Versuch $\mathrm{K} 13$ waren sie über die ganze Wandoberfläche verteilt. Für die Darstellung des Rissbildes wurde der Versuch K13 gewählt.

Der Bruch erfolgte duktil. Die Last erreichte bei etwas stärkerer Zunahme der Schiebungen einen Maximalwert und fiel dann progressiv ab. Es öffneten sich klaffende Risse (Bild 22). 
$45^{\circ}$ Fugenneigung:

Die Schiebungen nahmen bis zum Auftreten von Rissen linear mit der Last zu (Bild 12a). Die Schubsteifigkeit der Wand war geringer und die Schiebungen des Steins waren nur wenig grösser als beim Versuch mit $22.5^{\circ}$ Fugenneigung. Der Anteil des Steins an den Schiebungen betrug noch $40 \%$. Die übrigen Verzerrungsgrössen waren klein. Schon kurz nach Beginn der Belastung zeigten sich im Randbereich der Wand Risse, die den Lagerfugen (Bild 15) folgten. Es gab einige wenige Risse in Steinen. Bei ungefähr 50\% der Bruchlast begannen, ausgehend von Stossfugen, Risse in den beiden Kontaktflächen einer Lagerfuge derart aufeinander zuzuwachsen, dass sie das dazwischen liegende Fugenstück von den Steinen lösten (Bild 23). Bemerkenswert an diesen weniger als einen halben Stein langen und oft unzusammenhängenden Rissen war, dass die Rissöffnung von der Stossfuge weg abnahm, bis der Riss von blossem Auge nicht mehr wahrnehmbar war (Bild 22).

Der Bruch erfolgte ausgesprochen duktil. Bei praktisch konstant bleibender Last nahmen die Schiebungen sehr stark zu.

$67.5^{\circ}$ Fugenneigung:

Bis zum Auftreten von Rissen waren die Verzerrungen klein (Bild 13a). Bei ungefähr $30 \%$ der Bruchlast zeigten sich vor allem an der einen freien Wandkante feine Anrisse in der Lagerfuge. Die Schiebungen nahmen nun progressiv mit der Last zu. Ueberall über die Wand verteilt entstanden feine Risse (Bilder 9 und 15). Die Lagerfugen rissen bei weiter steigender Last sukzessive ganz durch. Ueberraschend öffnete sich ein treppenförmiger Riss in Wandmitte (Bild 22), und die Last fiel augenblicklich auf ungefähr $70 \%$ der Höchstlast. Der Riss wuchs bis auf Millimetergrösse an. Bei der Auswertung des Versuchs konnte gezeigt werden, dass die Dehnungen in $y$-Richtung im Rissbereich verschwindend klein waren, und dass es sich somit um ein Abgleiten in den Stossfugen handelte (Bild 23). Mit dem weiteren Oeffnen des Risses traten in den Krafteinleitungen beträchtliche Schiebungen auf. Die Versuchswand begann sich durch die Rückstellkräfte der Elastomerplatten in der Belastungseinrichtung zu verkeilen. Dadurch konnte die Last wieder weit über die ursprüngliche Höchstlast gesteigert werden. Der Versuch wurde dann abgebrochen. Die Laststufen 14 bis 16 sind im Last-VerzerrungsDiagramm (Bild 13a) nicht dargestellt. 
$90^{\circ}$ Fugenneigung:

Bei $20 \%$ der Bruchlast bildeten sich Risse in den Lagerfugen. Die Steifigkeit des Versuchskörpers in y-Richtung nahm deutlich ab. Die gemessenen Dehnungen der Steine waren verschwindend klein ( $B i l d$ 14a). Es zeigten sich Risse an den Stirnseiten des Versuchskörpers (Bild 15).

Der Bruch erfolgte mit dem Abplatzen der äusseren Steinlamelle im Bereich der Stossfugen (Bild 22).

\subsubsection{Verhalten der weiteren Versuchsserien}

\section{$0^{0}$ Fugenneigung:}

Das Tragverhalten der Versuchskörper der Serien 3 und 6 entsprach demjenigen des Versuchs der Standardserie gut ( $B i l d$ 10b). Sogar die Versuchskörper aus Kalksandsteinen zeigten ein sehr ähnliches Bild der Last-Verzerrungs-Beziehung (Bild 10e). Die Versuchskörper der Serien 4 und 5 wiesen eine viel geringere Steifigkeit des Steins im Mauerwerk auf. Dies dürfte auf die geringere Festigkeit des Steins zurückzuführen sein, die dann auch zu einer geringeren Steifigkeit der Wand führte. Der Anteil des Steins an der Verformung der Wand war ungefähr gleich wie beim Standardversuch. Beim Versuch der Serie 5 wurde die Steifigkeit zusätzlich durch den verlängerten Mörtel vermindert. Bei diesem Versuch traten die ersten Risse früher auf.

In der Versuchsserie 8 wurde ein Sichtstein von guter Qualität und hoher Druckund Querzugfestigkeit verwendet. Dieser Stein zeigte im Mauerwerk ein sehr steifes Verhalten (Bild 10c). Die Mauerwerksfestigkeit war dementsprechend höher als diejenige der Standardserie. Besonders bemerkenswert jedoch ist, dass diese Versuchswand bis zum Bruch kaum Risse aufwies. Das Bruchverhalten war ausgesprochen spröde.

Sehr interessant verhielt sich die Wand der Serie 10 aus vorfabriziertem Mauerwerk; sie wies praktisch keine Fugenverformung auf (Bild 10d). Trotzdem der Stein ein dem Standardversuch ähnliches Verformungsverhalten zeigte, war die Wand steifer. Bis zum Bruch der Wand bildeten sich kaum Risse. Der Bruch erfolgte spröde durch Spalten der Wand parallel zur Wandebene (Bild 24). 
Etwas erstaunlich war die relativ geringe Steifigkeit des Versuchskörpers der Serie 7. Der in dieser Serie verwendete Schalldämmstein, der praktisch ais Vollstein zu werten ist, verhielt sich im Mauerwerk erwartungsgemäss ausgesprochen steif. Der Versuchskörper hatte zwar die doppelte Anzahl Lagerfugen, da aber das Sichtmauerwerk der Serie 8 dieselbe Anzahl Lagerfugen aufwies, kann das abweichende Verhalten nicht mit der Anzahl Lagerfugen begrïndet werden.

\section{$22.5^{\circ}$ Fugenneigung:}

Da der Versuch mit $0^{\circ}$ Fugenneigung der Serie 3 gut mit dem Versuch der Standardserie 2 übereinstimmte, erstaunte es, dass bei $22.5^{\circ}$ Fugenneigung erhebliche Abweichungen im Last-Verzerrungs-Verhalten festzustellen waren (Bild 11b). Genau wie beim Versuch der Standardserie waren nur verschwindend kleine Dehnungen der Wand in $y$-Richtung sowie Dehnungen der Steine in $x$ - und $y$-Richtung festgestellt worden. Auch stimmten die Schubsteifigkeiten der Steine in den Versuchen beider Serien gut überein. Der Versuchskörper der Serie 3 wies jedoch in $x$-Richtung und auch bei den Schiebungen eine wesentlich grössere Steifigkeit auf, resp. der Anteil der Fugen an den Schiebungen betrug weniger als $20 \%$.

Bei allen Versuchen mit $22.5^{\circ}$ Fugenneigung waren die Dehnungen in $y$-Richtung praktisch null (Bild 11). Die Dehnungen in x-Richtung des Steins entsprachen im Unterschied zu den Versuchen der Serien 2 und 3 ungefähr demjenigen Anteil an der Dehnung des Versuchskörpers, der aufgrund der Erfahrungen mit den $0^{\circ}$-Versuchen zu erwarten war. Die Schiebungen der Wand entsprachen bei allen Versuchen (ausgenommen Serie 7) denjenigen der Steine. Der Schalldämmstein der Versuchsserie 7 zeigte in den Versuchen mit $22.5^{\circ}$ Fugenneigung nicht mehr das ausgesprochen steife Verhalten wie im $0^{\circ}$-Versuch (Bild 11f). Bei der Wand der Serie 7 zeigten sich Risse auch früher als bei den anderen.

\section{$45^{\circ}$ und $67.5^{\circ}$ Fugenneigung:}

Ein bemerkenswerter Unterschied zur Versuchsserie 2 bestand darin, dass bei allen Versuchsserien (ausgenommen Serie 7) die auf dem Stein gemessenen Schiebungen ungefähr den Schiebungen der Wand entsprachen (Bilder 12 und 13). Bei den $45^{\circ}$ Versuchen zeigten sich fast immer neben den Rissen entlang der Lagerfugen treppenförmige Risse, welche beim Mauerwerk mit sichtbarer Vermörtelung in der Stossfuge noch besser in Erscheinung traten. Bei den Versuchen mit $67.5^{\circ}$ Fugenneigung bildeten sich Risse vor allem entlang von Lagerfugen (Bild 18). Der Bruch erfolgte bei allen Versuchen mit $67.5^{\circ}$ Fugenneigung (ausgenommen Serien 2 und 9) durch Abgleiten entlang einer, meistens entlang zweier Lagerfugen. 
Bei den Versuchen mit Schalldämmsteinen erfolgte der Bruch bei beiden Fugenneigungen plötzlich. Die Wände hatten jeweils bis zum Bruch kaum Risse. Bei den Versuchen mit Kalksandsteinen rissen meistens ganze Fugen auf einmal durch. Dabei fiel die Last jeweils stark ab und konnte nicht wieder gesteigert werden.

\section{$90^{\circ}$ Fugenneigung:}

Bei allen Versuchen wurden im Gegensatz zu Serie 2 auch auf den Steinen Verformungen in $y$-Richtung festgestellt (Bild 14). Beim Modulstein der Serie 3 waren diese Verformungen jedoch ausserordentlich klein. Beim Sichtmauerwerk wurden die Verformungen hundertprozentig vom Stein erbracht. Den Lagerfugen entlang bildeten sich im Bereich der Stossfugen feine Risse, die aber ausser beim Schal1dämm-Mauerwerk nie zusammenwuchsen. An den Stirnseiten des Versuchskörpers bildeten sich Risse, die den Kraftverlauf und damit den Einfluss der Stossfugenvermörtelung sehr gut zeigten (Bild 19). Der Bruch erfolgte durch Ausbrechen der äusseren Steinlamellen.

\subsection{Steifigkeiten}

Aus den Last-Verzerrungs-Diagrammen wurden mit den folgenden Beziehungen Steifigkeitswerte für die Versuchskörper abgeschätzt:

$$
E_{x}=\frac{\sigma_{x}}{\epsilon_{x}}, \quad E_{y}=\frac{\sigma_{y}}{\epsilon_{y}}, \quad G_{x y}=\frac{\tau_{x y}}{\gamma_{x y}}
$$

Bei diesen Steifigkeiten, die für das Mauerwerk in der Tabelle 6 und für vermauerte Steine in der Tabelle 7 zusammengestellt sind, handelt es sich um Sekantenmoduli. Für die Ermittlung dieser Werte wurden Sekanten derart an die LastVerzerrungs-Kurven gelegt, dass die Kurve in einem möglichst grossen Lastbereich gut angenähert wurde. Die obigen Beziehungen stellen die Steigungen der betreffenden Sekanten dar. Die Spannungen sind auf den Bruttoquerschnitt bezogen. 


\subsection{Rissweiten}

In den Bildern 20 und 21 wurden beobachtete Rissweiten dargestellt. Im Bild 20 sind Mittelwerte aus allen pro Laststufe gemessenen Rissweiten gegen die Last aufgetragen. Im Bild 21 sind die jeweils grössten gemessenen Rissweiten aufgeführt. Die Anzahl der zur Verfügung stehenden Werte je Versuch und Laststufe war aus bereits erläuterten Gründen unterschiedlich. Es gab zudem Versuche, bei denen keine Rissmessungen vorgenommen werden konnten, weil bis zum Bruch kaum Risse vorhanden waren, oder, weil der Versuchskörper nur in Randzonen Risse aufwies, die für das Rissverhalten nicht repräsentativ waren.

In den Darstellungen wurde zwischen Rissen in Steinen und Rissen in den Fugen unterschieden, wobei nur Risse in den Lagerfugen gemessen werden konnten.

\subsection{Bruchlasten}

In der Tabelle 5 sind die aus den Versuchsresultaten ermittelten Bruchlasten, in denen ein gewisser Zeiteinfluss enthalten ist, aufgeführt. Im Verlauf der 20 bis 30 Minuten dauernden Messungen fiel die Last infolge Relaxation des Mauerwerkkörpers auf einen gewissen Wert ab, der gemessen und als Endlast $F_{E}$ registriert wurde. Die Bruchlasten wurden aus Endlasten ermittelt. Bei einem duktilen Verhalten der Mauerwerkswand konnten im Versuch mit dem beschriebenen Verfahren der Versuchssteuerung (Vorgabe von Verformungszielen) Messungen im Bereich des Lastmaximums durchgeführt werden. Aus weiteren Informationen wie Aufzeichnungen der beim Belasten kurzzeitig erreichten Lastspitzen und Protokollen der Versuchssteuerung konnten Lastmaxima gut abgeschätzt werden. Die Lastmaxima bei anschliessender Entfestigung des Versuchskörpers wurden als Bruchlasten definiert. Die Festlegung des Bruchzustandes war in wenigen Fällen, insbesondere bei Fugenversagen, problematisch, da die Krafteinleitungen eine scheinbare Verfestigung des Versuchskörpers vortäuschen konnten. In diesen Fällen musste aus dem Zerstörungsbild des Versuchskörpers abgeschätzt werden, ob die Tragfähigkeit der Mauerwerkswand als erschöpft gelten konnte.

Bei eher sprödem Verhalten des Versuchskörpers trat der Bruchzustand plötzlich und oft mit ganzer oder teilweiser Zerstörung des Versuchskörpers ein. Trat der 
Bruch kurz vor Erreichen der nächsten Laststufe oder bei Messbeginn ein, wurde der Lastabfall infolge Relaxation des Mauerwerkkörpers abgeschätzt und die Bruchlast aus den bei der Belastung erreichten Lastspitzen ermittelt.

\subsection{Isomodu]-Verbandmauerwerk}

Beim Isomodul-Verbandmauerwerk sind die Lagerfugen nicht vollflächig vermörtelt (vg1. Kap. 2). Demzufolge waren die Spannungen in diesen Versuchswänden nicht gleichmässig verteilt. Bei tiefen Lasten entstanden deshalb Zwängungsrisse, die das Mauerwerk parallel zur Oberfläche in verschiedene Schalen aufspalteten. Die Risse bedingten grosse Verformungen der Versuchskörper quer zur Wandebene. Diese Verformungen wurden durch die Krafteinleitungen behindert, so dass sich die äusseren Schalen wölbten. Beim Versuch mit $0^{\circ}$ Fugenneigung führte dies schliesslich zu einem Ausknicken dieser Schalen. Der Einfluss der ungleichmässigen Spannungsverteilung auf das Trag- und das Rissverhalten ist schwierig abzuschätzen. Der Aussagewert der Versuche mit Verbandmauerwerk ist somit entsprechend zu werten.

\subsection{Streuung der Versuchsresultate}

Da je Versuchsserie und Fugenneigung nur ein einziger Versuch durchgeführt worden ist, ist es angebracht, noch einige Ueberlegungen zur Streuung der Resultate anzustellen. Im Rahmen der Versuchsserie 2 wurden mit den Fugenneigungen $22.5^{\circ}$ und $45^{\circ}$ je drei Versuche zur Beobachtung der Streuung durchgeführt. Später wurden der Versuchsserie 2 noch sechs Versuche mit RILEM-Versuchskörpern angehängt (vg1. Anhand B), wobei drei der Versuche ohne Deformetermessungen erfolgten. Es ist sowohl auf die labormässige Herstellung der Versuchskörper als auch auf die sorgfäitige Durchführung der Versuche zurückzuführen, dass kaum wesentliche Streuungen in den Resultaten festgestellt werden konnten. Die Versuche verhielten sich bei der Wiederholung in bezug auf die Verformung prinzipiell gleich. Beim Rissverhalten waren einige Unterschiede festzustellen. Dies betraf insbesondere den Rissbeginn und die Verteilung der Risse über die Wandoberfläche. Das Rissverhalten beeinflusste zwar das Verformungsverhalten, doch wurde dieser Einfluss erst bei Lasten nahe der Bruchlast massgebend. Ueber die Streuung der Bruchlasten der je drei identischen Versuche der Serie 2 geben die Tabellen 5 und B2 (im Anhang B) Auskunft. 


\section{VORSCHLAG FUER RECHENWERTE DER MAUERWERKSFESTIGKEIT}

\subsection{Bruchbedingung für Mauerwerk}

Am Institut für Baustatik und Konstruktion wurde eine Bruchbedingung für Mauerwerk unter ebenem Spannungszustand entwickelt [2], [3]. Die Bruchbedingung dient als Grundlage für die Bemessung von Mauerwerkswänden unter vertikaler und horizontaler Belastung in der Wandebene [5], [6] und wird durch die folgenden fünf Beziehungen (Regimes) festgelegt. Alle Grössen sind auf den Bruttoquerschnitt bezogen. Die $x$-Richtung steht senkrecht zu den Lagerfugen, die $y$-Richtung parallel dazu (Bild 7).

Zugversagen (Ausschluss von Zugspannungen):

(1) $\tau_{x y}^{2}-\sigma_{x} \cdot \sigma_{y} \leq 0$

Druckversagen:

(2) $\tau_{x y}^{2}-\left(\sigma_{x}+f_{M x}\right) \cdot\left(\sigma_{y}+f_{M y}\right) \leq 0$

(3) $\tau_{x y}^{2}+\sigma_{y} \cdot\left(\sigma_{y}+f_{M y}\right) \leq 0$

Gleiten der Lagerfugen:

(4) $\tau_{x y}^{2}-\left(c-\sigma_{x} \cdot \tan p\right)^{2} \leq 0$

Zugversagen (Trennbruch) in den Lagerfugen (Ausschluss von Zugspannungen):

(5) $\tau_{x y}^{2}+\sigma_{x} \cdot\left[\sigma_{x}+2 \cdot c \cdot \tan \left(\frac{\pi}{4}+\frac{p}{2}\right)\right] \leq 0 ; \sigma_{x} z-c \cdot \cos p$

Im dreidimensionalen Spannungsraum $\sigma_{x^{*}} \sigma_{y^{\prime}} \tau_{x y}$ wird die Bruchbedingung für Mauerwerk durch zwei elliptische Kegel $(1,2)$, zwei Kreiszylinder $(3,5)$ und eine Ebene (4) dargestellt. 
Die Bruchbedingung für Mauerwerk hat vier unabhängige Materialparameter:

$$
\begin{aligned}
& f_{M x} \text { Mauerwerksfestigkeit in x-Richtung } \\
& f_{\text {My }} \text { Mauerwerksfestigkeit in y-Richtung } \\
& \text { c Kohäsion der Lagerfuge } \\
& \text { p Reibungswinkel der Lagerfuge }
\end{aligned}
$$

Um die Beziehungen auf den einachsigen Druckversuch anwenden zu können, muss die Bruchbedingung auf die Hauptspannungen $\left(\sigma_{1}=0, \sigma_{2}=F_{u} / A\right)$ transformiert werden:

$$
\begin{aligned}
& \sigma_{x}=\sigma_{2} \cdot \cos ^{2} \alpha \\
& \sigma_{y}=\sigma_{2} \cdot \sin ^{2} \alpha \\
& \tau_{x y}=\sigma_{2} \cdot \sin \alpha \cdot \cos \alpha
\end{aligned}
$$

Mit $\alpha$ ist der Winkel der $x$-Achse zur 2. Hauptrichtung bezeichnet (Bild 7).

Es ergibt sich der Verlauf der einachsigen Druckfestigkeit $\sigma_{2}$ in Abhängigkeit des Winkels $\alpha\left[\sigma_{2}=f(\alpha)\right]$ :

- Aus der Beziehung (2) erhalten wir für $\alpha=0^{\circ}$

$$
\sigma_{2}=-f_{M x}
$$

- Für den Bereich $0<\alpha<\alpha_{G r}$ erhalten wir aus den Beziehungen (1) und (3):

$$
\begin{aligned}
& \sigma_{2}=-f_{M y} \\
& a_{G r}=\text { Winkel, der dem Uebergang ins Regime (4) Fugenversagen entspricht }
\end{aligned}
$$


- Für Fugenversagen, d.h. für $\alpha>a_{G r}$ ergibt die Beziehung (4)

$$
\sigma_{2}=\frac{-c}{\cos ^{2} \alpha \cdot(\tan \alpha-\tan \phi)}
$$

Diese Funktion $\sigma_{2}=f(\alpha)$ hat für $\alpha=\alpha_{\min }$ ein Minimum, $\sigma_{x}\left(\alpha_{\min }\right)=-c \cdot \cos p$. Der Uebergang zu Regime (5) wird bei $\alpha_{\min }$ erreicht:

$$
\alpha_{\min }=\frac{\pi}{4}+\frac{p}{2}
$$

- Im Bereich $\alpha_{\min }<\alpha<90^{\circ}$ ist Regime (5) massgebend. Die einachsige Druckfestigkeit verbleibt auf dem konstanten Wert:

$$
\sigma_{2}=-2 \cdot c \cdot \tan \alpha_{\min }
$$

- Für $\alpha=90^{\circ}$ gilt theoretisch: $\sigma_{2}=-f_{M y}$

\subsection{Parameterwerte aus den Versuchsresultaten}

In diesem Abschnitt werden die Versuchsresultate für die Bestimmung der vier Parameterwerte der beschriebenen Bruchbedingung für Mauerwerk verwendet. Im vorangehenden Abschnitt 5.1 wurde die einachsige Druckfestigkeit $\sigma_{2}$ als Funktion des Fugenneigungswinkel $\alpha$ ausgedrückt. Es wurden Versuchswände mit Fugenneigungswinkeln $\alpha=0^{\circ}, 22.5^{\circ}, 45^{\circ}, 67.5^{\circ}$ und $90^{\circ}$ geprüft. Die 2. Hauptrichtung entspricht der Vertikalen, d.h. der Richtung der angreifenden Last (Bild 7). Dividiert man die jweilige Bruchlast mit der Bruttofläche senkrecht zur 2. Hauptrichtung, resultiert die einachsige Druckfestigkeit $\sigma_{2}$. Der Verlauf der einachsigen Druckfestigkeit ist also in fünf Punkten ermittelt. Demzufolge stehen genügend Gleichungen für die Bestimmung der, vier Parameter der Bruchbedingung zur Verfügung.

\section{Steinversagen:}

$$
\sigma_{2}\left(\alpha=0^{\circ}\right)=f_{M x} ; \quad \sigma_{2}\left(\alpha=22.5^{\circ}\right)=f_{M y}
$$




\section{Fugenversagen:}

Es muss verifiziert werden, dass $\alpha_{G r}<45^{\circ}$ ist. Wenn dies der Fall ist, stehen zwei Gleichungen für die Bestimmung der zwei Unbekannten c (Kohäsion der Lagerfuge) und $p$ (Reibungswinkel der Lagerfuge) zur Verfügung. Sollte sich herausstellen, dass $\alpha_{\min }<67.5^{\circ}$ ist, muss $\sigma_{2}\left(\alpha=67.5^{\circ}\right)$ in die Gleichung für das Regime (5) eingesetzt werden.

Die aus den Versuchsresultaten ermittelten Parameter $f_{M x^{\prime}} f_{M y^{\prime}}$ c und $\rho$ sind in der Tabelle 8 zusammengestellt.

\subsection{Unsicherheiten bei der Bestimmung der Parameter}

In der beschriebenen Bruchbedingung [3] werden Zugspannungen konsequent ausgeschlossen. In Wirklichkeit weist das Mauerwerk jedoch eine geringe Zugfestigkeit auf. Aus der Arbeit von Ganz [2] ist ersichtlich, dass die verschiedenen Bereiche (Regime) der Bruchbedingung von der Zugfestigkeit unterschiedlich beeinflusst werden. Bild 28 zeigt schematisch den Einfluss der Zugfestigkeit auf die einachsige Festigkeit $\sigma_{2}(\alpha)$. Die Bestimmung der Mauerwerksfestigkeit in $y-R i c h-$ tung aus einachsigen Versuchen mit $22.5^{\circ}$ Fugenneigung ist somit mit einer gewissen Unsicherheit behaftet. Für die einachsigen Versuche mit $90^{\circ}$ Fugenneigung sollte theoretisch $\sigma_{2}\left(\alpha=90^{\circ}\right)=f_{M y}$ gelten. Wie die Resultate zeigen, tritt bei diesen Versuchen sehr früh ein Versagen der Haftung der Lagerfugen mit dem Stein ein, wodurch eine teilweise Abtragung der Last über die Lagerfugen nicht mehr möglich ist und demzufolge die ganze Last über die Stossfugen abgetragen werden muss. Damit wird die Gestaltung der Stossfuge über Gebühr massgebend. Eine teilweise Vermörtelung der Stossfuge verursacht zum Beispiel Probleme bezüglich der Kraftausbreitung im Stein.

Für die Bestimmung von $f_{M y}$ sollten deshalb zweiachsige Versuche mit einer Fugenneigung $\alpha=90^{\circ}$ durchgeführt werden, wobei die Druckspannung senkrecht zu den Lagerfugen klein sein darf $\left(\sigma_{1} / \sigma_{2} \leq 1 / 10\right)$. Es wäre z.B. möglich, RILEM-Körper um $90^{\circ} \mathrm{zu}$ kippen und und in $x$-Richtung mit einer geringen äusseren Vorspannung, aufgebracht mit Spannstangen, zu versehen. Die Kräfte in den Spannstangen müssten beobachtet werden, aber sonst wäre der Aufwand nur wenig grösser als derjenige für die RILEM-Versuche. 
Nur indirekt im Zusammenhang mit der Zugfestigkeit steht ein anderes Problem, das sich bei der Bestimmung der Fugenparameter $c$ und $p$ der Bruchbedingung stellt. Wie beschrieben, zeigte sich bei einigen Versuchen mit $45^{\circ}$ und $67.5^{\circ}$ Fugenneigung eine Versagensart der Fugen, die als Gleiten der Stossfugen bezeichnet wurde. Sie ist vermutlich von den bei einigen Mauerwerksarten nicht voll vermörtelten Stossfugen verursacht worden. Hinzu kommt, dass die Seitenflächen der Steine im Bereich der Stossfugen viel glatter sind als die im rohen Zustand geschnittenen Flächen entlang der Lagerfugen, wodurch die Haftung des Mörtels in der Stossfuge entsprechend geringer ist. Es ist anzunehmen, dass bei diesen Mauerwerksarten die Schubtragfähigkeit in den Stossfugen geringer ist als in den Lagerfugen. Dies hat eine ungleichmässige Spannungsverteilung in der Lagerfuge und schliesslich den im Kapitel 4 beschriebenen treppenförmigen Trennbruch zur Folge.

Für die vorgestellte Bruchbedingung wurde als einzige Fugenversagensart Gleiten der Lagerfugen angenommen. Für diese Versagensart gibt die modifizierte Bruchbedingung von Coulomb ein zutreffendes Mode11. Darin werden eine Ebene und und eine Zylinderschale eingeführt. Der Reibungswinkel p beeinflusst die Neigung, und der zweite Parameter c bestimmt die Lage der Ebene im Spannungsraum bezüglich der $\tau_{x y}$-Achse. Wenn diese zwei Parameter aus den Versuchsresultaten bestimmt werden, führt dies möglicherweise in einigen Fällen zu einer Unterschätzung, insbesondere der Kohäsion c. Dies könnte die Erklärung dafür sein, dass die mit den Abscherversuchen (Anhang $B$ ) bestimmte Kohäsion $c$ ein wenig grösser war. Es soll hier aber auch angemerkt werden, dass der Einfluss der Fugenparameter auf den Bemessungswert einer mit Schub beanspruchten Wand gering ist.

\subsection{Mauerwerks- und Steinfestigkeiten}

Es erscheint interessant, die Tragfähigkeit des Mauerwerks direkt aus Festigkeitswerten der Komponenten Stein und Mörtel herzuleiten. Beim Verbundbaustoff Mauerwerk treten jedoch Wechselwirkungen zwischen diesen Komponenten auf. Die vermauerten Steine und der vermauerte Mörtel zeigen ein anderes Tragverhalten. Die vorliegenden Versuche haben keine Beziehungen zwischen den Mauerwerksfestig-

keiten $f_{M x}$ und $f_{M y}$ und der Druckfestigkeit $f_{S}$ oder der Querzugfestigkeit $f_{q z}$ des Steins erkennen lassen. Die Bestimmung der Druckfestigkeit und der Querzugfestigkeit des Steins sollen daher als reine Qualitätsprüfungen verstanden werden. 
Um ein in der Anwendung einfaches und damit sichereres Bemessungsverfahren zu erhalten, ist es überzeugender, Kennwerte für die Festigkeit des Mauerwerks als solche für die einzelne Komponente zu suchen. Die Bruchbedingung berücksichtigt die wesentlichen Eigenschaften von Mauerwerk unter ebener Beanspruchung wie Orthotropie und Inhomogenität direkt. Die Parameter der Bruchbedingung können mit einfach durchzuführenden Mikroversuchen (siehe Anhang B) bestimmt werden. 


\section{ABSCHLIESSENDE BETRACHTUMGEN}

In diesem Kapitel werden qualitative Angaben zum Tragverhalten gemacht und Gedanken für eine mögliche Verbesserung des Baustoffs Mauerwerk dargelegt.

\subsection{Qualitative Beschreibung des Tragverhaltens}

In diesem Abschnitt wird versucht, den Stand der Erkenntnisse zusammenzufassen und das Tragverhalten anhand der einachsigen Versuche qualitativ zu beschreiben. Die Betrachtung beginnt mit einem Mauerwerkskörper, der senkrecht zur Lagerfuge beansprucht wird. Die weniger steife Mörtelfuge erfährt im Vergleich zum Stein grössere Querdehnungen und dadurch bauen sich in der Fuge Druckspannungen und im Stein Zugspannungen quer zur Kraftrichtung auf. Die Steine werden infolge dieser Querzugspannungen gespalten, und es bilden sich folglich Risse normal zur Lagerfuge. Die Wand wird schlussendlich meist parallel zu ihrer Oberfläche gespalten.

Es ist schwierig, den Einfluss des Lochanteils und des Lochbildes des Steins auf das Tragverhalten zu erfassen. Einen Anhaltspunkt gibt die Querdehnungsprüfung des Steins. Die Wirkung der Fuge wird durch eine Elastomerplatte mit definierten Eigenschaften simuliert. Bei Steinen mit ungefähr gleichem Lochbild und vor allem gleichem Lochanteil ist eine relativ gute Korrelation mit der Mauerwerksfestigkeit $f_{M x}$ festzustellen. Es wird ersichtlich wie komplex das Zusammenwirken der beiden Komponenten im Mauerwerk ist. Der Stein setzt den durch die Fugen verursachten Querzugsspannungen einen Widerstand entgegen, welcher aus den durch Tonqualität und Herstellung bedingten Festigkeitseigenschaften der Steinmatrix resultiert. Im weiteren wird die Querdehnung der Mörtelfuge nicht nur durch die Spannungsverteilung in der Fuge beeinflusst, sondern auch durch die Festigkeitseigenschaften, die der Mörtel im Mauerwerk erlangt. Der Mörte1 kann im Mauerwerk nicht ungestört abbinden. Durch das von Kapillarporen im Stein verursachte mehr oder weniger starke Saugen des Steins wird der Abbindeprozess des Zementes im Mörte 1 beeinträchtigt. Laut Grandet [9], der Mörtelfugen mit der Röntgendiffraktometrie und mit einem Elektronenmikroskop untersuchte, läuft die Hydratation des Zementes infolge des Wasserentzuges nicht vollständig ab. Neuere Untersuchungen versuchen, Zusammenhänge zwischen der Kristallstruktur des Zementgels und dem PH-Wert herzustellen. Damit wäre es denkbar, aus dem PH-Wert des Mörtels, der z.B. durch das Saugen des Steins beeinflusst sein könnte, auf die Festigkeitseigenschaften der Fuge zu schliessen. 
Weicht die Richtung der Beanspruchung von der Vertikalen zur Lagerfuge ab, nimmt die Wirkung des Querdehnungsvorgangs mit zunehmender Neigung gegenüber der Lagerfuge rasch $a b$, da auch die Grösse der Spannung in x-Richtung rasch abnimmt. Die Schubspannungen in der Wand werden mit zunehmender Neigung der Wirkungsachse der Last grösser. Die Stossfugen beeinflussen nun zunehmend den Spannungsfluss durch die Wand. Sie weisen wegen des schlechteren Verbundes eine geringere Fähigkeit zur Uebertragung von Schubspannungen auf als die Lagerfugen. Dadurch wird nach Untersuchungen an der Technischen Hochschule Darmstadt [11] die Spannungsverteilung im Stein und in der Lagerfuge beeinflusst. Bei teilweiser Vermörtelung konzentrieren die Stossfugen die Spannungen in die vermörtelten Bereiche. Eine geschickte Anordnung der Löcher im Stein und ein geringerer Lochantei 1 begünstigen die Kraftausbreitung. Durchgehende Längslamellen wie beim Stein der Serie 2, die sich auf die Vermörtelung in der Stossfuge abstützen, führen zu einer Konzentration der Spannungen auf diesen beiden inneren Lamellen.

Wird die Neigung der angreifenden Last bezüglich der Lagerfuge grösser als ungefähr $40^{\circ}$, tritt ein Abgleiten oder Aufreissen der Fugen auf. Dabei wird entweder die Haftung des Mörtels am Stein gelöst oder der Mörtel im Kontaktbereich zum Stein abgeschert. Bei Fugenversagen sind die Festigkeitseigenschaften des Mörtels im Mauerwerk massgebend. Diese Festigkeitseigenschaften werden durch das Saugen des Steins wie beschrieben beeinflusst.

Der oft diskutierte Einfluss der Makroverzahnung der Mörtelfuge in den Löchern des Steins auf den Verbund besteht nicht in der Erhöhung der Tragfähigkeit, sondern in einer Verbesserung des Tragverhaltens. Zur Mobilisation des Widerstandes dieser Mörtelzapfen sind grössere Verformungen notwendig. Ist der Haftverbund zwischen Stein und Mörtel überwunden, bewirken die Mörtelzapfen ein duktileres Verhalten mit grosser Verformbarkeit bei praktisch gleichbleibender Last. Die Versuche mit dem Schalldämmstein haben gezeigt, dass sich die kleinen Löcher und demzufolge das Fehlen der Mörtelzapfen auf die Tragfähigkeit nicht nachteilig auswirken, dass aber das Bruchverhalten durch den plötzlichen Lastabfall beim Versagen des Verbundes spröde ist.

Die durch die Stossfugen verursachte ungleichmässige Spannungsverteilung in den Lagerfugen kann zum Auftreten von Zugspannungen in der einen Hälfte der Lagerfuge jedes Steins führen. Dies erzeugt einen Trennbruch der Lagerfuge wie anhand eines Fotos im Bild 23 gezeigt. Es tritt ein treppenförmiges Abgleiten über die Stossfugen auf (Bilder 9 und 22). 
Schliesslich soll noch der Fall der Beanspruchung parallel zu den Lagerfugen (Neigungswinkel $90^{\circ}$ ) betrachtet werden. Bei ganzflächiger Vermörtelung der Stossfugen werden die Steine durch die Querdehnung des Mörtels auf Querzug beansprucht. Bei teilweiser Vermörtelung wird ein bedeutender Teil der Last indirekt über die Lagerfugen in die benachbarten Steinlagen abgetragen. Löst sich die Haftung zwischen Stein und Lagerfugen, wirkt die gesamte Last auf die Stossfugen. Die teilweise Vermörtelung führt zu Spannungskonzentration und Spaltung der Steine.

\subsection{Anregungen zu Verbesserungen}

In diesem Abschnitt sind einige Gedanken festgehalten, die vor allem der Verbesserung des Produktes Mauerwerk dienen können. Wie die vorgestellte Bruchbedingung gut zeigt, tritt das Versagen meistens im Stein auf. Das Lochbild der Steine kann im Hinblick auf den Kräftefluss durch die Stossfugen weiterentwickelt werden. Eine Erhöhung der Festigkeit der Steine erscheint nicht sinnvoll, umsomehr als dies in der Schweiz mangels geeigneter Tonvorkommen nicht einfach sein dürfte. Die vorangehenden Betrachtungen sollen verdeutlichen, dass eingedenk der Wechselwirkungen zwischen den beiden Komponenten des Mauerwerks Verbesserungen nicht nur am Stein, sondern insbesondere an den Mörtelfugen vorgenommen werden sollten. Für die Ausführung der Fugen kann aus den Versuchen geschlossen werden, dass für eine gute Schubtragfähigkeit des Mauerwerks eine vollflächige Vermörtelung am günstigsten ist. Ferner sind Massnahmen interessant, die die Festigkeit des Mörtels im Mauerwerk erhöhen. Die Versuche mit vorfabriziertem Mauerwerk haben gezeigt, dass mit einem Stein von eher durchschnittlicher Festigkeit Mauerwerksfestigkeiten erreicht werden können, die sonst nur Mauerwerk der Sonderqualität (z.B. Sichtmauerwerk) aufweist (vgl. Tabelle 8). Das "Geheimnis" des vorfabrizierten Mauerwerks kann nur in der Mörtelmischung 1iegen. Möglicherweise wurde durch die Zugabe von Filler die spezifische innere Oberfläche des Mörtels derart verbessert, dass durch das damit verbesserte Wasserrückhaltevermögen des Mörtels der Abbindeprozess des Zementes im Mauerwerk verbessert und somit die Festigkeit des Mörtels im Mauerwerk erhöht wurde. Es soll aber hier auch darauf hingewiesen werden, dass bei höheren Mauerwerksfestigkeiten ein sprödes, d.h. verformungsarmes, Bruchverhalten der Wände auftreten kann. 
Aus Gründen wie Einsparung von Zement, einseitige Ausrichtung auf gute Verarbeitbarkeit, Vereinfachung des Bauprozesses, usw., werden oft Abstriche bei der Mörtelqualität oder der Ausführung der Fugen, speziell der Stossfugen, akzeptiert. Insofern dürfen die Versuche der Serie 5 dieses Berichtes mit verlängertem Mörte1, welche einen fast unbedeutenden Abfall in der Festigkeit des Mauerwerks gegenüber Zementmörtel gezeigt haben, nicht unbesehen als Rechtfertigung für ein solcher Vorgehen herangezogen werden. In den Versuchen wurde nämlich ein Stein mit niederer Festigkeit mit verlängertem Mörtel vermauert. Ein bedeutender Festigkeitsabfall von bis zu $30 \%$ tritt jedoch auf, wenn ein Stein mit höherer Festigkeit mit verlängertem Mörtel kombiniert wird, wie dies der RILEM-Versuch R15 gezeigt hat (Anhang B).

\subsection{Vorschläge für das weitere Vorgehen}

Viele Einflussgrössen auf die Festigkeitswerte und das Tragverhalten von Mauerwerk können dank der Erkenntnisse dieser Forschungsarbeit besser verstanden werden. Um für künftige Forschungsprojekte nützliche Anknüpfungspunkte zu schaffen, sollen in diesem Abschnitt einige durch die vorliegende Arbeit offengelegte Fragen angeführt werden.

Da es sich beim Schalldämmstein praktisch um einen Vollstein handelt, wäre aufgrund theoretischer Ueberlegungen zu erwarten, dass die Mauerwerksfestigkeit in $x$ - und $y$-Richtung ungefähr gleich sind. Der Versuch mit $90^{\circ}$ Fugenneigung bestätigte diese Annahme. Da die Stossfugen beim Schalldämmstein voll vermörtelt worden sind, kann die Mauerwerksfestigkeit in y-Richtung aus diesem Versuch ermittelt werden. Es ergibt sich ein leicht grösserer Wert als für die Festigkeit in $x$-Richtung. Dies ist damit erklärbar, dass die Stossfugen den weit grösseren Abstand haben als die Lagerfugen. Der Versuch mit $22.5^{\circ}$ Fugenneigung erreichte jedoch weniger als die Hälfte der Festigkeit in $x$-Richtung. Es ist noch unklar, womit diese (scheinbare) Unstimmigkeit erklärt werden kann.

Weitere Forschungsbemühungen sollten den Wechselwirkungen zwischen Stein und Fuge gewidmet werden. Durch Erforschung der Einflüsse, die auf die Festigkeitseigenschaften des Mörtels im Mauerwerk wirken, können die Grundlagen für die Entwicklung besserer Mörtelmischungen geschaffen werden. Die Schwierigkeiten 
eines derartigen Forschungsvorhabens dürften darin liegen, dass Kenntnisse aus verschiedenen Gebieten wie Chemie, Kristallographie, Festkörperphysik und Materialtechnologie zusammengeführt werden müssten. Zur Bestimmung der Festigkeitswerte können einfache Kleinversuche wie sie im Anhang B beschrieben wurden herangezogen werden. 


\section{ZUSAMMENFASSUNG}

Zur Bemessung von Schubwänden aus Mauerwerk wurde am Institut für Baustatik und Konstruktion eine Bruchbedingung für ein Wandelement unter ebenem Spannungszustand entwickelt. Um für die vorhandene Vielfalt von Steinen und Mörteln die massgebenden Parameter dieser Bruchbedingung experimentell zu bestimmen, wurden die in diesem Bericht beschriebenen Versuche durchgeführt. Aus den Resultaten der Forschungsarbeit wurden Rechenwerte für die Festigkeiten von Mauerwerk aus Backstein und Kalksandstein bestimmt.

Es wurden quadratische Scheiben mit $1.2 \mathrm{~m}$ Kantenlänge einachsig bis zum Bruch belastet. Die mehrheitlich ca. $150 \mathrm{~mm}$ starken Wände waren derart gemauert, dass die Senkrechte auf die Lagerfugen zur Kraftrichtung die Winkel $0^{\circ}, 22.5^{\circ}, 45^{\circ}$, $67.5^{\circ}$ und $90^{\circ}$ aufwies. Für die 11 Versuchsserien wurden 11 verschiedene Steine und 3 verschiedene Mörtelmischungen verwendet. Aus jeder solchen Versuchsserie liessen sich die vier Parameter der Bruchbedingung bestimmen.

In den Versuchen wurden die Lasten und die Verformungen der Wände beobachtet. Es zeigte sich, dass das Tragverhalten von Mauerwerk massgeblich durch Wechselwirkungen der zwei Komponenten Steine und Mörtel charakterisiert wird. Die Zusammenhänge sind dabei ziemlich komplex. Die Steine und der Mörtel zeigten im Verbund ein Verhalten, das sich nicht aus den Stein- und Mörtelfestigkeiten herleiten liess. Die Ausführung der Fugen, vor allem der Stossfugen, erweist sich für die Schubtragfähigkeit als besonders wichtig. Wird nicht die ganze Fugenfläche vermörtelt, konzentrieren sich die Spannungen in den vermörtelten Bereichen. Die Steine werden dadurch ungleichmässig beansprucht, was sich ungünstig auf die Tragfähigkeit auswirkt. Die Stossfugenflächen der Backsteine sind, bedingt durch die Herstellung, relativ glatt und weisen einen schlechteren Verbund mit dem Mörtel auf als die Lagerfugenflächen. In den Versuchen zeigte sich dies besonders deutlich als Abgleiten in den Stossfugen.

Für die verschiedenen Ausführungen der Steine hinsichtlich Lochbild, Lochanteil und Abmessungen liessen sich aus den Versuchen keine markanten Unterschiede im Tragverhalten aufzeigen. Gewisse Einflüsse wurden im Zusammenhang mit der Kraftübertragung in den Stossfugen festgestellt. Eine günstige Anordnung der Löcher und ein geringer Lochanteil fördern die Ausbreitung der Kraft im Stein bei teilweiser Vermörtelung der Stossfugen. 
Um Materialeinsparungen und Vereinfachungen im Vermauern zu erzielen werden oft verlängerte Mörtel verwendet, bei denen ein Teil des Zementes durch hydraulischen Kalk ersetzt ist. Solche Mörtel weisen eine bedeutend geringere Festigkeit auf als Zementmörtel. In den Versuchen war ein Abfall der Mauerwerksfestigkeit bei Verwendung von verlängertem Mörtel an Stelle von Zementmörtel kaum feststellbar. Dies erklärt sich aus der Tatsache, dass in den Versuchen mit dem verlängerten Mörtel ein Stein geringer Festigkeit verwendet wurde. Wird hingegen ein Stein höherer Festigkeit mit verlängertem Mörtel vermauert, muss mit einem Festigkeitsabfall bis zu $30 \%$ gerechnet werden. Zudem zeigte sich bei Versuchen mit vorfabriziertem Mauerwerk, dass mit einer geeigneten Mörtelmischung und Steinen durchschnittlicher Festigkeit ein Mauerwerk mit hoher Festigkeit hergestellt werden kann.

Werden zur Erzielung höherer Mauerwerksfestigkeiten Steine mit hoher Festigkeit verwendet, muss mit sprödem Bruchverhalten gerechnet werden. Eine wichtige Aufgabe der Fugen ist, dem Mauerwerk Verformbarkeit zu geben. Es scheint somit interessant zu sein, Mörtelmischungen zu entwickeln, die ein Mauerwerk mit höherer Festigkeit und genügender Verformbarkeit ergeben. 


\section{RESUME}

Pour établir les bases du dimensionnement des parois en maçonnerie supportant un effort tranchant, un critère de rupture, se rapportant à un élément de parois sollicité selon un plan, a été déterminé à l'Institut de statique et de construction de I'EPF à Zurich. Le but des investigations expérimentales décrites dans ce rapport est d'examiner l'influence des caractéristiques des divers produits existants en maçonnerie sur ce critère de rupture. Des valeurs de calcul de la résistance à la rupture de la maçonnerie en terre cuite ou en silico-calcaire ont été finalement extraites de ces essais.

Les murs d'essais, mesurants $1.2 \mathrm{~m} \times 1.2 \mathrm{~m}$, étaient soumis à un effort de compression simple jusqu' à l'état de rupture. Les murs, dont la plupart avaient une épaisseur d'environ $150 \mathrm{~mm}$, ont été maçonnés de façon que la perpendiculaire sur les lits de pose soit inclinée de $0^{\circ}, 22.5^{\circ}, 45^{\circ}, 67.5^{\circ}$ ou $90^{\circ}$ par rapport à la direction de la force. Pour les 11 séries d'essais, 11 briques et trois types de mortier différents ont été choisis. Chaque série complète permit de detérminer les quatres paramètres du critère de rupture.

Durant les essais les charges et les déplacements ont été mesurés. Il fut constaté que le comportement des murs en maçonnerie était fortement influencé par l'interdépendance qui existe entre les briques et le mortier. Les relations sont ainsi très complexes et le comportement des ouvrages en maçonnerie ne peut pas etre dérivé en considérant seulement la résistance de ces deux composantes. La résistance à l'effort tranchant dépend fortement de la conception des joints en général et des joints verticaux en particulier. Les contraintes se concentrent dans les parties de la section transversale des joints remplies de mortier. En suite les briques sont chargées irrégulièrement ce qui diminue la résistance à la rupture. La surface de contact dans les joints verticaux des briques en terre cuite est relativement lisse à cause de la méthóde de fabrication. La liaison avec le mortier est ainsi inférieure à celle qui agit à la base des briques. Cette différence se manifeste très distinctement par l'apparition du glissement des joints verticaux. Les différentes conceptions des briques en ce qui concerne la disposition et le pourcentage des alvéoles ainsi que la dimension des briques ne conduisaient pas à des variations prononcées dans le comportement des murs d'essais. Toutefois une certaine influence leur est attribuée dans la transmis- 
sion des efforts au travers des joints verticaux. Une disposition favorable et un petit pourcentage d'alvéoles améliore la propagation des efforts dans les briques lorsque les joints verticaux ne sont pas remplis de mortier.

Pour économiser le matérial et pour faciliter le maçonnage on prend souvent du mortier "batard", dans lequel une portion du ciment est remplacée par de la chaux. Ces mortiers "bátard" ont une résistance beaucoup plus faible que les mortiers au ciment, mais la diminution de la résistance de la maçonnerie lors de leur utilisation dans les essais était insignifiante. Cela provient du fait que dans les essais que des briques de faible résistance ont été jointes avec ce mortier. Par contre, si l'on utilise une brique de haute résistance avec ce mortier "batard", une diminution de la résistance de la maçonnerie d'environ $30 \%$ peut survenir. Par ailleurs il a été constaté lors d'essais d'une maçonnerie préfabriquée, qu'une brique de résistance moyenne jointe avec un mortier de type favorable peut donner une maçonnerie à haute résistance.

Si on utilise des briques à haute résistance pour augmenter la résistance de la maçonnerie, la rupture peut atre très brusque. Une fonction importante des joints est de rendre la maçonnerie moins rigide et $i 1$ peut etre très intéressant de développer de nouvelles sortes de mortiers avec lesquels il serait possible de fabriquer une maçonnerie à haute résistance, avec une ductilité convenable. 


\section{SUMMARY}

For the design of masonry shear walls a failure criterion for biaxially loaded masonry elements was developed at the Institute of Structural Engineering of the Swiss Federal Institute of Technology (ETH) in Zürich. To experimentaliy study the important parameters of this failure criterion for the various existing masonry products, the tests described in this report were conducted. Values for the calculation of the strength of masonry with clay and sand-lime bricks were then derived.

The test specimens were square of $1.2 \mathrm{~m}$ length and mostly about $150 \mathrm{~mm}$ thick. They were loaded uniaxially up to failure. The specimens were built up in a way that the normal to the bed joints was inclined at $0^{\circ}, 22.5^{\circ}, 45^{\circ}, 67.5^{\circ}$ and $90^{\circ}$ in relation to the direction of the load. In the 11 test series 11 different bricks and 3 types of mortar were used. Out of each complete serie the four parameters of the failure criterion could be determined.

During the test the loads and deformations were observed. The behaviour of the masonry walls was essentially characterized by the interaction between the two components brick and mortar. The relationship appeared rather involved. The behaviour of the masonry could not be determined from the strength of bricks and mortar. The details of the joints, especially of the perpend joints influenced considerably the ultimate shear carrying capacity. If not the whole joint is filled with mortar, the stresses concentrate in the mortar-filled parts of the joints. This causes a non-uniform stress distribution in the bricks and a decrease in the resistance. The surface of the clay bricks adjacent to the perpend joints is relatively smooth due to the manufacturing process and therefore the bond with the mortar in the perpend joints is weaker than in the bed joints. In the tests this led to sliding in the perpend joints.

The behaviour of the test walls was hardly influenced by the different geometrical patterns of a brick. Some of these parameters had a certain influence on the force transmission through the perpend joints. A favourable arrangement and a small cross-sectional area of the cores in hollow bricks improve the propagation of the force within the brick in case of perpend joints filled only partially with mortar. 
Besides mortar with cement as bonding agent, mortars with lime as partial cement substitute were often used to reduce the costs of mortar and to simplificate the placing of the mortar. Such "cement/lime/sand mortars" have a reduced strength compared to cement mortars. In the tests with such a mortar the reduction of the masonry strength was not significant. This can be explained by the fact that in these tests a brick of lower strength was used with a "cement/lime mortar". However, if a high-strength brick is used with such a mortar a reduction of the masonry strength of about $30 \%$ can result. Tests with prefabricated masonry moreover showed that with a appropriate mortar composition and with bricks of ordinary strength, a high-strength masonry can be obtained.

Masonry with high-strength bricks will generally fail in a sudden manner. An important function of the mortar joints is to provide to the masonry some ductility. Thus, it seems to be of interest to develop new types of mortar leading to high-strength masonry with a improved ductility. 


\section{VERDANKUNGEN}

\section{Die Verfasser danken}

der Kommission zur Förderung der wissenschaftlichen Forschung (KWF), Bern und dem Verband Schweiz. Ziegel- und Steinfabrikanten (VSZS), Zürich

herzlich für die grosszügige Finanzierung des Forschungsprogrammes. In diesen Dank schliessen sie die Mitglieder der Beratenden Kommission, die Herren Dr. R. Furler, H. Gubler, W. Nydegger, E. Reinle, W. Santi, E. Setz und C. Weder, ein. Besonders hervorzuheben ist die anfängliche Beratung und Betreuung des Projektes durch Herrn Dr. H.R. Ganz, vormals Mitarbeiter des Institutes.

Die Durchführung der Versuche oblag dem Prüf- und Forschungsinstitut des VSZS in Sursee. Herrn Prof. Dr. E. Amrein und dem technischen Leiter, Herrn M. Schmid, allen Mitarbeitern des $P+F$, insbesondere den Herren Gisler, Graber und Korner, gebührt der Dank für eine in jeder Beziehung erfreuliche Zusammenarbeit.

Die Auswertung der Versuche und die Ausarbeitung des Berichtes wurden am Institut für Baustatik und Konstruktion der ETH Zürich durchgeführt. Die Verfasser danken Herrn R. Caflisch und seinem Nachfolger, Herrn Dr. B. Zimmerli, für die Betreuung des Projektes, Herrn M. Baumann, dip1. Bauing. ETH, für die messtechnische Beratung, den Herren G. Göseli und L. Sieger und Frau S. Burki für die Gestaltung des Berichtes und Herrn K. Bucher für die fachkundige Anfertigung einiger Geräte.

Für die kostenlose Lieferung der Elastomerlager sei der Proceq AG, Zürich. bestens gedankt. 


\section{LITERATURVERZEICHNIS}

[1] Ganz H.R.. Thürlimann B., Versuche über die Festigkeit von zweiachsig beanspruchtem Mauerwerk, Institut für Baustatik und Konstruktion. ETH Zürich, Versuchsbericht Nr. 7502-3, Februar 1982, Birkhäuser Verlag, Basel (ISBN 3-7643-1339-0)

[2] Ganz H.R.., Mauerwerkscheiben unter Normalkraft und Schub, Institut für Baustatik und Konstruktion, ETH Zürich, Bericht Nr. 148, September 1985. Birkhäuser Verlag, Basel (ISBN 3-7643-1782-5)

[3] Ganz H.R., Thürlimann B., Bruchbedingung für zweiachsig beanspruchtes Mauerwerk, Institut für Baustatik und Konstruktion, ETH Zürich, Bericht Nr. 143, September 1984, Birkhäuser Verlag, Basel (ISBN 3-7643-1666-7)

[4] Ganz H.R., Thürlimann B., Versuche an Mauerwerksscheiben unter Normalkraft und Querkraft, Institut für Baustatik und Konstruktion, ETH Zürich, Versuchsbericht Nr. 7502-4, Mai 1984, Birkhäuser Verlag, Base 1 (ISBN 3-7043-1665-9)

[5] Ganz H.R., Thürlimann B., Plastic Strength of Masonry Shear Walls, 7th International Brick Masonry Conference, Melbourne, February 1985

[6] Ganz H.R., Thürlimann B., Shear Design of Masonry Walls, Proceedings of Structural Engineering Congress 85, ASCE, Chicago, September 1985

[7] Furler R., Tragverhalten von Mauerwerkswänden unter Druck und Biegung, Institut für Baustatik und Konstruktion, ETH Zürich, Bericht Nr. 109. Februar 1981, Birkhäuser Verlag, Base1 (ISBN 3-7643-1257-2)

[8] Schwartz J.. Thürlimann B., Versuche über die Rotationsfähigkeit von Zementsteinmauerwerk. Institut für Baustatik und Konstruktion, ETH Zürich, Versuchsbericht Nr. 8401-1, September 1986, Birkhäuser Verlag, Base 1 (ISBN 3-7643-1850-3)

[9] Grandet J.. Contribution à l'Etude de la Liaison entre la Pate de Ciment Portland et les Terres Cuites, Université Paul Sabatier de Toulouse, Thèse, Avri1 26, 1971

[10] Schweizerischer Ingenieur- und Architekten-Verein (SIA), Zürich, Norm SIA 177 "Mauerwerk", 1980

[11] Mann W., Müller H., Cracking Characteristics of Transversely Loaded Brick Masonry in Theory and Practice. Proceedings of the 5 th International Brick Masonry Conference, Washington, October 1979

[12] RILEM Commission 24-BW Murs porteurs et Maçonnerie "Recommandations générales pour les Méthodes d'Essais des Murs porteurs", Recommandation provisoire de la Réunion International des Laboratoires d'Essais et de Recherches sur les Matériaux et les Constructions (RILEM), Paris 


\section{BEZEICHNUNGEN}

\section{Geometrische Grössen}

$x \quad$ Koordinatenachse senkrecht zu den Lagerfugen

y Koordinatenachse parallel zu den Lagerfugen

$u \quad$ Verschiebung in $x$-Richtung

$v \quad$ Verschiebung in $y-R i c h t u n g$

w Rissweite

$\bar{w} \quad$ mittlere Rissweite

$w_{\max }$ maximale Rissweite

A Bruttoquerschnittsfläche

a Neigungswinkel der Lagerfugen

\section{Kraftgrössen}

F Belastung

$F_{u} \quad$ grösste Last, Bruchlast

$F_{A} \quad$ Last vor den Verformungsmessungen

$F_{E} \quad$ Last nach den Verformungsmessungen

\section{Materialwerte, Spannungen}

$E_{x} \quad$ Sekantensteifigkeit in $x$-Richtung

$E_{y} \quad$ Sekantensteifigkeit in $y$-Richtung

$G_{x y}$ Schubsteifigkeit

$f_{s} \quad$ Druckfestigkeit des Steins

$f_{q z} \quad$ Querzugfestigkeit des Steins

$f_{M x} \quad$ Mauerwerksfestigkeit in $x$-Richtung

$f_{M y}$ Mauerwerksfestigkeit in $y$-Richtung

c Kohäsion der Lagerfugen

p Reibungswinkel der Lagerfugen 
$\begin{array}{ll}\sigma_{x} & \text { Spannung in } x-R i c h t u n g \\ \sigma_{y} & \text { Spannung in } y-R i c h t u n g \\ \tau_{x y} & \text { Schubspannung } \\ \sigma_{1} & \text { grössere Hauptspannung } \\ \sigma_{2} & \text { kleinere Hauptspannung }\end{array}$

Verformungsgrössen

$E_{x} \quad$ Verzerrung in $x$-Richtung

$E_{y} \quad$ Verzerrung in $y-R i c h t u n g$

$\gamma_{x y} \quad$ Schiebung

$\dot{\epsilon}^{x} \quad$ Verzerrungsgeschwindigkeit

Allgemeine Bezeichnungen

K Versuchsbezeichnung 'Kleinkörper'

$R \quad V e r s u c h s b e z e i c h n u n g$ 'RILEM' 


\begin{tabular}{|c|c|c|c|c|c|c|c|c|}
\hline \multirow{2}{*}{$\begin{array}{l}\text { Vers.- } \\
\text { ser 1e }\end{array}$} & \multicolumn{5}{|c|}{ stein } & \multirow{2}{*}{ Mortel } & \multirow{2}{*}{$\begin{array}{l}\text { Mand- } \\
\text { starke }\end{array}$} & \multirow{2}{*}{$\begin{array}{l}\text { Bemerk- } \\
\text { ungen }\end{array}$} \\
\hline & $\begin{array}{l}\text { Grund- } \\
\text { mater ial }\end{array}$ & Lochb1ld & $\begin{array}{l}\text { Loch- } \\
\text { antel } 1\end{array}$ & Format & $\begin{array}{l}\text { Stoss- } \\
\text { fuge }\end{array}$ & & & \\
\hline 2 & B & DLS & 468 & Hodul & Pvs & Zement & $150 \mathrm{~mm}$ & $\begin{array}{l}\text { Standart- } \\
\text { ser } 10\end{array}$ \\
\hline 3 & B & vL & $46 \%$ & Modul & PVS & Zement & $150 \mathrm{~mm}$ & \\
\hline 4 & $\begin{array}{c}B \\
\text { niedris }\end{array}$ & VL & 408 & Mormal & HFV & Zement & $150=$ & \\
\hline 5 & $\begin{array}{c}\text { B } \\
\text { niedrig }\end{array}$ & VL & $40 x$ & Normal & HFV & Verl. M. & $150 \mathrm{man}$ & \\
\hline 6 & $\begin{array}{c}\text { B } \\
\text { hoch }\end{array}$ & VL & 408 & Normal & HFV & Zement & $150-m$ & \\
\hline 7 & B & $\begin{array}{l}\text {-Voll- } \\
\text { stein }\end{array}$ & $\begin{array}{c}k 1 e \text { in } \\
12 \%\end{array}$ & $1 / 2$ M. & VFv & Zement & $175 \mathrm{~mm}$ & Caimo \\
\hline 8 & B & DLS & 358 & $1 \%, \mathrm{M}$ & VFv & Zement & $140=0$ & $\begin{array}{l}\text { Sicht- } \\
\text { stein }\end{array}$ \\
\hline 9 & B & DLS & Isol.? & Modul & & Zement & $325 \mathrm{~mm}$ & Isomodul \\
\hline 10 & B & DLS & 458 & Normal & Pvs & Gussm. & $150=$ & $\begin{array}{l}\text { Vor ger. } \\
\text { MW. }\end{array}$ \\
\hline 11 & $\mathrm{~K}$ & $\mathbf{R L}$ & $20 \%$ & Normal & HFV & Zement & 145 an & $\begin{array}{l}\text { Kalk- } \\
\text { sandstein }\end{array}$ \\
\hline 12 & K & MDRL & $20 \%$ & Mormal & KFV & Zement & $145=$ & $\begin{array}{l}\text { Kalk- } \\
\text { sandstein }\end{array}$ \\
\hline
\end{tabular}

Tabelle 1: Variation der Einflussparameter

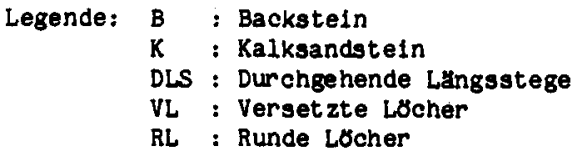

NDRL: Nicht durchgehende, runde Locher PVS : Pressfuge mit verdecktem 'Spatz' HFV : Halbe Fugenfluche vermortelt VFV : Volle Fugenfluche vermortelt

\begin{tabular}{|c|c|c|c|c|c|c|}
\hline \multirow[t]{2}{*}{$\begin{array}{l}\text { Vers - } \\
\text { serie }\end{array}$} & \multicolumn{5}{|c|}{$\begin{array}{l}\text { Zuordnung der Versuche zu dem } \\
\text { Fugennelgungswinkel a }\end{array}$} & \multirow[t]{2}{*}{$\begin{array}{l}\text { RILEY- } \\
\text { versuche }\end{array}$} \\
\hline & $\alpha=0^{\circ}$ & $22.5^{\circ}$ & $45^{\circ}$ & $67.5^{\circ}$ & $90^{\circ}$ & \\
\hline 2 & K 62 & $\begin{array}{ll}K & 13 \\
K & 14 \\
K & 15\end{array}$ & $\begin{array}{ll}\mathbf{x} & 16 \\
\mathrm{~K} & 17 \\
\mathrm{~K} & 18\end{array}$ & × 63 & K 53 & $\begin{array}{l}1,2,3 \\
\text { R } 4,5,6\end{array}$ \\
\hline 3 & K 19 & K 20 & & & K 54 & R 7 \\
\hline 4 & K 21 & K 22 & K 23 & K 24 & K 55 & R 8 \\
\hline 5 & K 25 & K 26 & K 27 & $\times 28$ & K 56 & \\
\hline 6 & K 29 & K 30 & K 31 & K 32 & K 57 & \\
\hline 7 & K 33 & K 34 & × 35 & K 36 & K 58 & R 10 \\
\hline 8 & K 37 & K 38 & & & K 59 & R 11 \\
\hline 9 & $\times 39$ & $\times 40$ & K 41 & K 42 & & \\
\hline 10 & K 43 & K 44 & & & & \\
\hline 11 & K 45 & K 46 & $\times 47$ & K 48 & K 60 & R 12 \\
\hline 12 & $\times 49$ & K 50 & K 51 & K 52 & K 61 & R 13 \\
\hline
\end{tabular}

Tabelle 2: Ver suchsprogramm wit den Bezelchnungen 


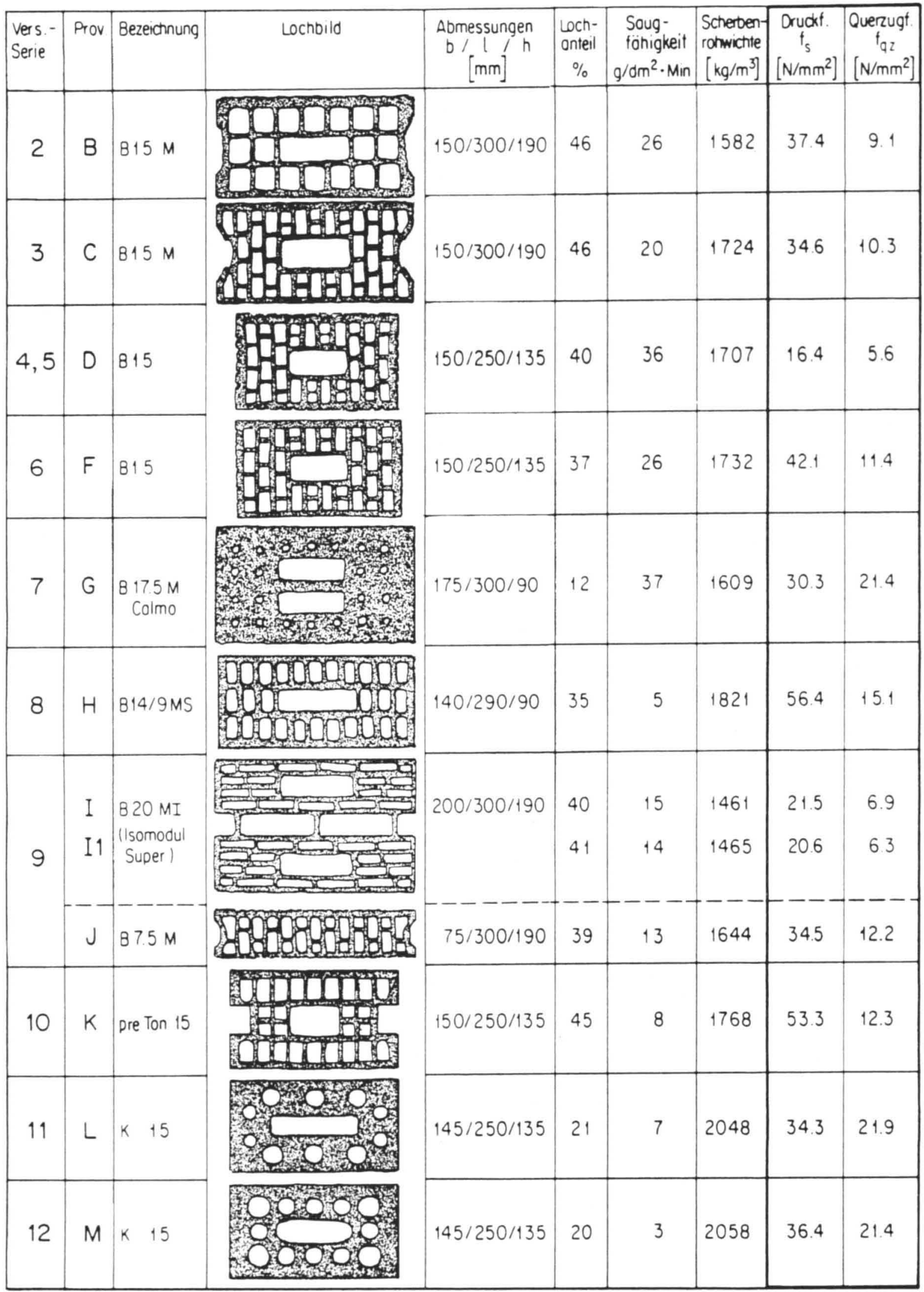

Tabelle 3: Normprüfung der Steine 


\begin{tabular}{|c|c|c|c|c|c|}
\hline $\begin{array}{l}\text { Vers.- } \\
\text { ser le }\end{array}$ & $\begin{array}{l}\text { Bindealttel- } \\
\text { gehalt } \\
\qquad\left[\mathrm{kg} / \mathrm{m}^{3}\right]\end{array}$ & $\begin{array}{l}\text { Prof- } \\
\text { alter } \\
\text { [Tage }]\end{array}$ & $\begin{array}{c}\text { Raungewicht } \\
\text { (erhärteter } \\
\text { Mortel) } \\
{\left[\mathrm{kg} / \mathrm{m}^{\prime}\right]}\end{array}$ & $\begin{array}{r}\text { Biegezug } \\
\text { festigkeit } \\
{\left[\mathrm{N} / \mathrm{mm}^{2}\right]}\end{array}$ & $\begin{array}{l}\text { Druck- } \\
\text { festigke1t } \\
\qquad\left[\mathrm{N} / \mathrm{mm}^{2}\right]\end{array}$ \\
\hline $\begin{array}{c}2 \\
3 \\
4 \\
5 \\
6 \\
7 \\
8 \\
9 \\
10 \\
11 \\
12 \\
\text { RILEM }\end{array}$ & $\begin{array}{c}365 \mathrm{PC} \\
345 \mathrm{PC} \\
347 \mathrm{PC} \\
100 \mathrm{PC} / 250 \mathrm{HK} \\
349 \mathrm{PC} \\
350 \mathrm{PC} \\
350 \mathrm{PC} \\
350 \mathrm{PC} \\
\text { (Gussmortel) } \\
350 \mathrm{PC} \\
350 \mathrm{PC} \\
355 \mathrm{PC}\end{array}$ & $\begin{array}{l}33 \\
31 \\
30 \\
34 \\
34 \\
34 \\
35 \\
40 \\
32 \\
31 \\
34 \\
28\end{array}$ & $\begin{array}{l}2094 \\
2158 \\
2104 \\
2032 \\
2104 \\
2078 \\
2099 \\
2097 \\
2058 \\
2110 \\
2137 \\
2067\end{array}$ & $\begin{array}{l}6.4 \\
5.8 \\
5.1 \\
2.5 \\
5.3 \\
5.3 \\
5.3 \\
5.6 \\
6.0 \\
5.6 \\
5.4 \\
6.0\end{array}$ & $\begin{array}{r}27.9 \\
24.9 \\
22.3 \\
8.9 \\
22.4 \\
22.8 \\
22.5 \\
23.3 \\
29.6 \\
25.2 \\
25.0 \\
25.2\end{array}$ \\
\hline
\end{tabular}

Tabelle 4: Normprufung des Mortels

Legende: $\mathrm{PC}=$ Portland-Zement

HK = Hydraulischer Kalk

\begin{tabular}{|c|c|c|c|c|c|}
\hline \multirow{2}{*}{$\begin{array}{l}\text { Versuchs- } \\
\text { serie }\end{array}$} & \multicolumn{5}{|c|}{ Fugennel gung a } \\
\hline & $0^{\circ}$ & $22.5^{\circ}$ & $45^{\circ}$ & $67.5^{\circ}$ & $90^{\circ}$ \\
\hline $\begin{array}{r}3 \\
4 \\
5 \\
6 \\
7 \\
8 \\
9 \\
10 \\
11 \\
12\end{array}$ & $\begin{array}{r}-1414 \\
-1450 \\
-880 \\
-780 \\
-1465 \\
-1451 \\
-2735 \\
-1271 \\
-2300 \\
-1800 \\
-2061\end{array}$ & $\begin{array}{r}-471 \\
-455 \\
-419 \\
-600 \\
-373 \\
-330 \\
-780 \\
-637 \\
-1600 \\
-690 \\
-850 \\
-715 \\
-983\end{array}$ & $\begin{array}{l}-231 \\
-207 \\
-207 \\
-300 \\
-270 \\
-565 \\
-392 \\
-530 \\
-400 \\
-625\end{array}$ & $\begin{array}{l}-210 \\
-191 \\
-356 \\
-220 \\
-342 \\
-190 \\
-200\end{array}$ & $\begin{array}{l}-112 \\
-126 \\
-138 \\
-206 \\
-840 \\
-909\end{array}$ \\
\hline
\end{tabular}

Tabelle 5: Bruchlasten $F_{U}$ in $\mathrm{KN}$

\begin{tabular}{|c|r|r|r|r|r|r|}
\hline \multirow{2}{*}{$\begin{array}{c}\text { Versuchs- } \\
\text { ser 1e }\end{array}$} & \multicolumn{2}{|c|}{$E_{\mathbf{x}}$} & $E_{\mathbf{y}}$ & \multicolumn{3}{c|}{$\mathbf{G}_{\mathbf{x y}}$} \\
\cline { 2 - 7 } & \multicolumn{1}{|c|}{$\alpha=0^{\circ}$} & $22.5^{\circ}$ & $90^{\circ}$ & $22.5^{\circ}$ & $45^{\circ}$ & $67.5^{\circ}$ \\
\hline & & & & & & \\
2 & 7000 & 4100 & 1550 & 1050 & 900 & 1450 \\
& & & & 1100 & 1000 & \\
3 & 7700 & 9800 & 2050 & 1600 & 800 & \\
4 & 3100 & 2800 & 1350 & 900 & 1100 & 2800 \\
5 & 2100 & 2500 & 1800 & 800 & 950 & 850 \\
6 & 6400 & 7300 & 3400 & 1750 & 2100 & 1750 \\
7 & 3400 & 2800 & 4300 & 1400 & 2400 & 750 \\
8 & 16100 & 16000 & 10700 & 4800 & & \\
9 & 8200 & 11300 & & 1850 & 1700 & 1950 \\
10 & 12800 & 12900 & & 3800 & & \\
11 & 6900 & 8800 & 4300 & 3400 & 3200 & 2900 \\
12 & 10200 & 10100 & 6550 & 4400 & 4800 & 4000 \\
\hline
\end{tabular}

Tabelle 6: Steifigkeit des Mauerwerks in $\mathrm{N} / \mathrm{mm}^{2}$ 


\begin{tabular}{|c|r|r|r|r|r|r|}
\hline \multirow{2}{*}{$\begin{array}{l}\text { Versuchs- } \\
\text { ser1e }\end{array}$} & \multicolumn{2}{|c|}{$E_{\mathbf{X}}$} & $E_{\mathbf{y}}$ & \multicolumn{3}{c|}{$G_{\mathbf{x y}}$} \\
\cline { 2 - 7 } & \multicolumn{1}{|c|}{$\alpha=0^{\circ}$} & $22.5^{\circ}$ & $90^{\circ}$ & $22.5^{\circ}$ & $45^{\circ}$ & $67.5^{\circ}$ \\
\hline & & & & & & \\
2 & 9550 & 16300 & 18600 & 1850 & 1800 & 9450 \\
3 & 13600 & 54900 & 7550 & 1950 & & \\
4 & 4850 & 5950 & 3100 & 900 & 1000 & 5100 \\
5 & 4900 & 3900 & 2750 & 850 & 1100 & 1050 \\
6 & 14800 & 10100 & 5200 & 1900 & 2350 & 2000 \\
7 & 20800 & 8100 & 6950 & 2250 & 5300 & 4300 \\
8 & 25800 & 22400 & 10700 & 4900 & & \\
10 & 14800 & 17800 & & 4200 & & \\
11 & 10100 & 10700 & 9450 & 4400 & 4400 & 4050 \\
12 & 15700 & 19800 & 8250 & 5250 & 5050 & 4150 \\
\hline
\end{tabular}

Tabelle 7: Steifigkeit der Steine im Mauerwerk in $\mathrm{N} / \mathrm{mm}^{2}$

\begin{tabular}{|c|c|c|c|c|c|c|c|}
\hline \multirow{2}{*}{$\begin{array}{l}\text { Versuchs- } \\
\text { serie }\end{array}$} & \multicolumn{3}{|c|}{ Mauerwerk } & \multirow{2}{*}{$\begin{array}{c}\mathrm{f}_{\mathrm{MX}} \\
{\left[\mathrm{N} / \mathrm{mm}^{2}\right]}\end{array}$} & \multirow{2}{*}{$\begin{array}{c}\mathrm{P}_{\mathrm{Hy}} \\
{\left[\mathrm{N} / \mathrm{mm}^{2}\right]}\end{array}$} & \multirow{2}{*}{$\begin{array}{c}\mathrm{c} \\
{\left[\mathrm{N} / \mathrm{mm}^{2}\right]}\end{array}$} & \multirow[t]{2}{*}{$\tan$} \\
\hline & Steintyp & $\begin{array}{l}\text { Prove- } \\
\text { nienz }\end{array}$ & Mortel & & & & \\
\hline $\begin{array}{r}2 \\
3 \\
4 \\
5 \\
6 \\
7 \\
8 \\
9 \\
10 \\
11 \\
12\end{array}$ & $\begin{array}{llll}\text { B } & 15 & M \\
B & 15 & M \\
B & 15 \\
B & 15 \\
\text { B } & 15 \\
B & 17.5 & \\
\text { B } & 14 / 9 & \mathrm{M} & \mathrm{C} \\
\text { Isomodul } & \mathrm{S} . \\
\text { pre Ton } & 15 \\
\mathrm{~K} & 15 \\
\mathrm{~K} & 15\end{array}$ & $\begin{array}{c}B \\
C \\
D \\
D \\
F \\
G \\
H \\
I, J \\
K \\
L \\
M\end{array}$ & $\begin{array}{l}2 \\
2 \\
Z \\
v \\
2 \\
2 \\
2 \\
2 \\
c \\
2 \\
2\end{array}$ & $\begin{array}{r}7.86 \\
8.06 \\
4.89 \\
4.33 \\
8.14 \\
6.91 \\
16.28 \\
3.26 \\
12.78 \\
10.34 \\
11.84\end{array}$ & $\begin{array}{l}2.49 \\
3.33 \\
2.07 \\
1.83 \\
4.33 \\
3.03 \\
9.52 \\
1.77 \\
4.72 \\
4.11 \\
5.65\end{array}$ & $\begin{array}{l}0.17 \\
0.33 \\
0.30 \\
0.53 \\
0.27 \\
0.24 \\
0.27 \\
0.27\end{array}$ & $\begin{array}{l}0.71 \\
0.61 \\
0.60 \\
0.66 \\
0.71 \\
0.65 \\
0.77 \\
0.85\end{array}$ \\
\hline
\end{tabular}

Tabelle 8: Parameter der Bruchbedingung aus den Versuchen 


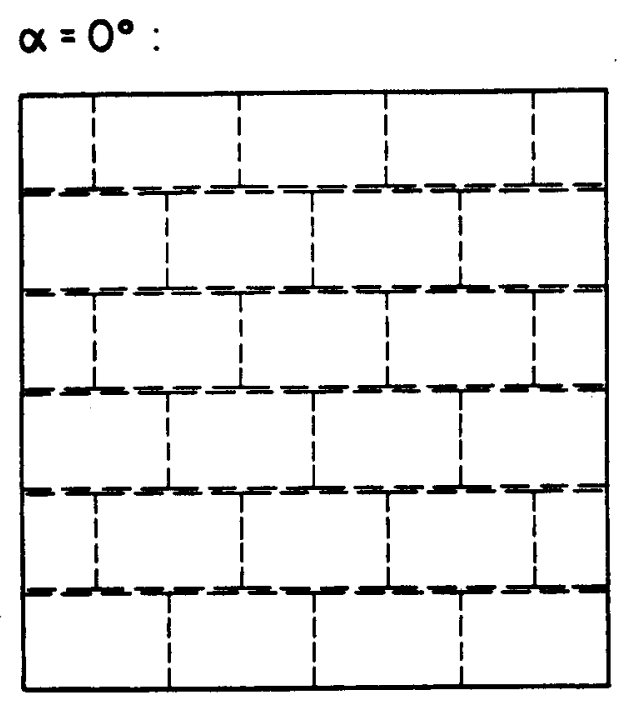

$\alpha=22.5^{\circ}$ :
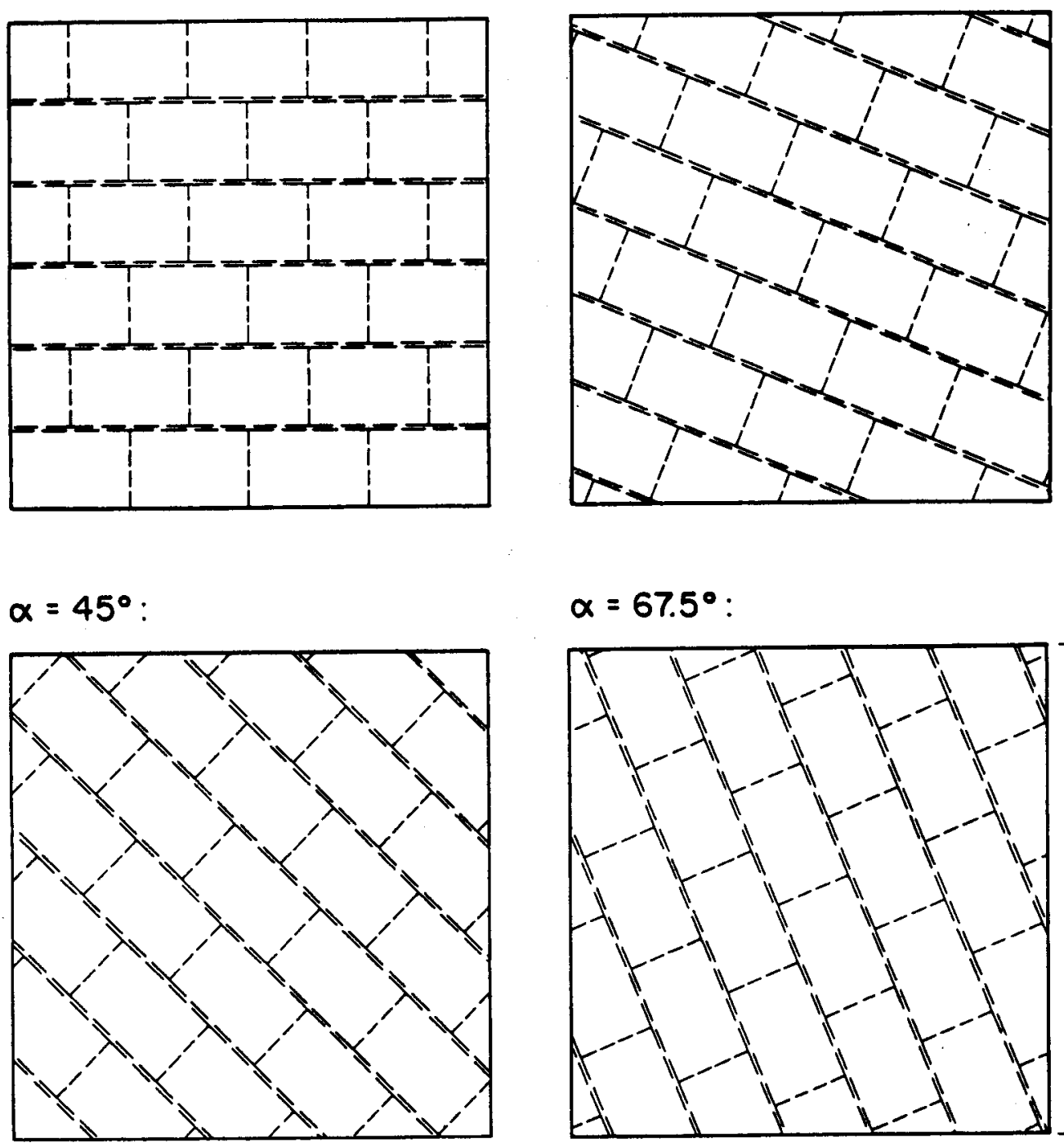

$\alpha=67.5^{\circ}$ :

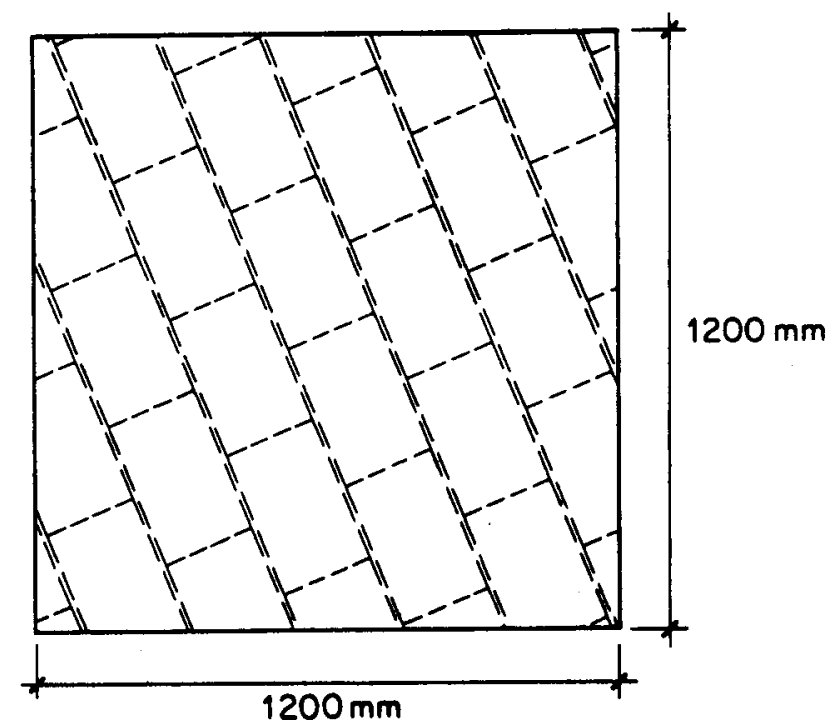

Versuchsserien: (1), 2, 3,9
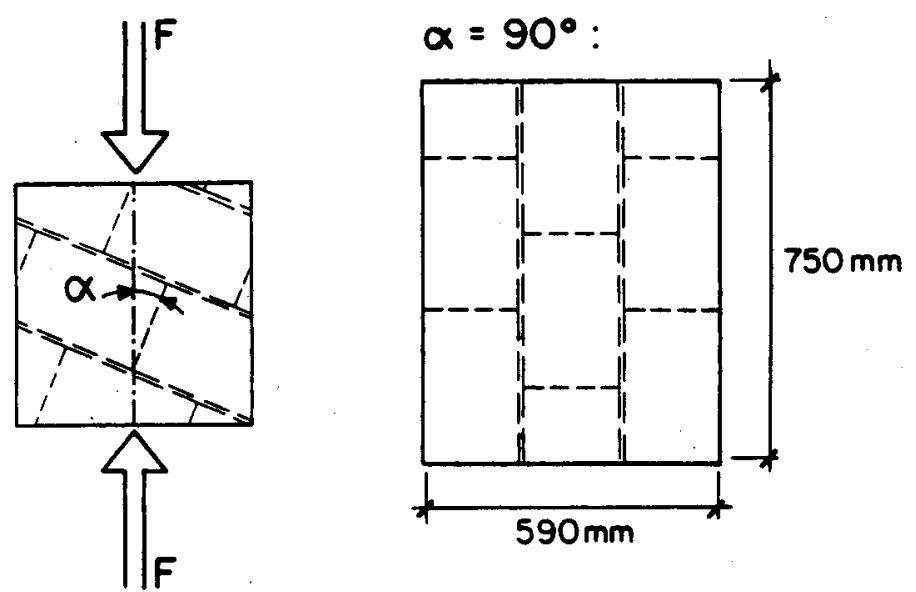

Bild 1 : Versuchskörper mit Modulsteinen 
$\alpha=0^{\circ}$ :

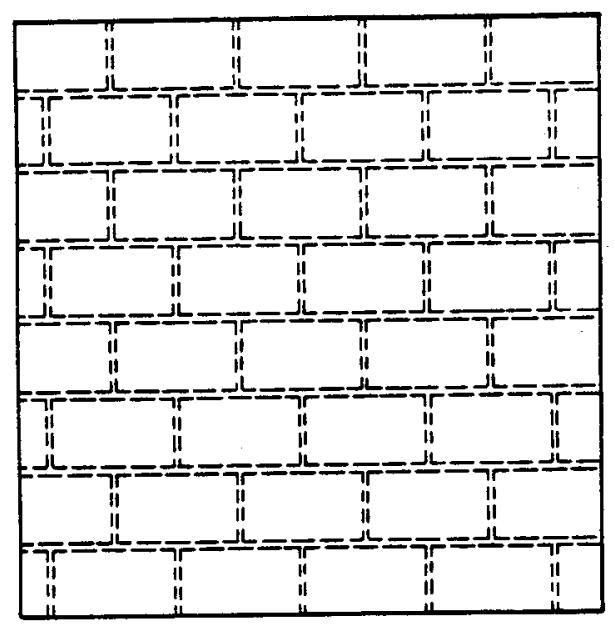

$\alpha=45^{\circ}$ :

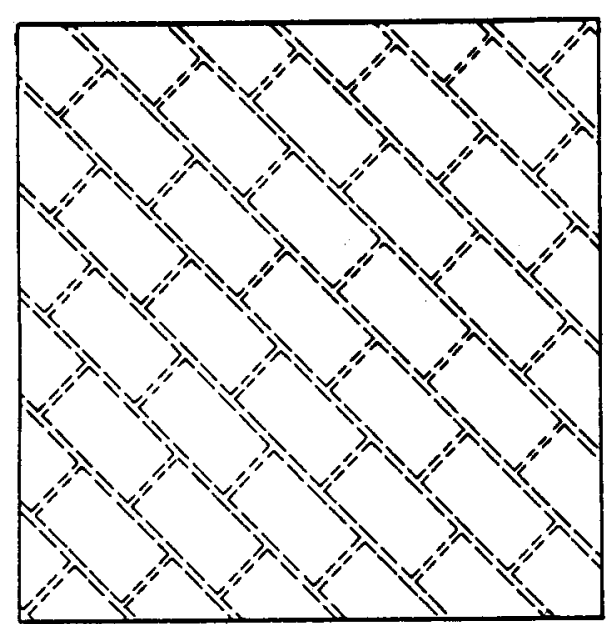

$\alpha=22.5^{\circ}$ :

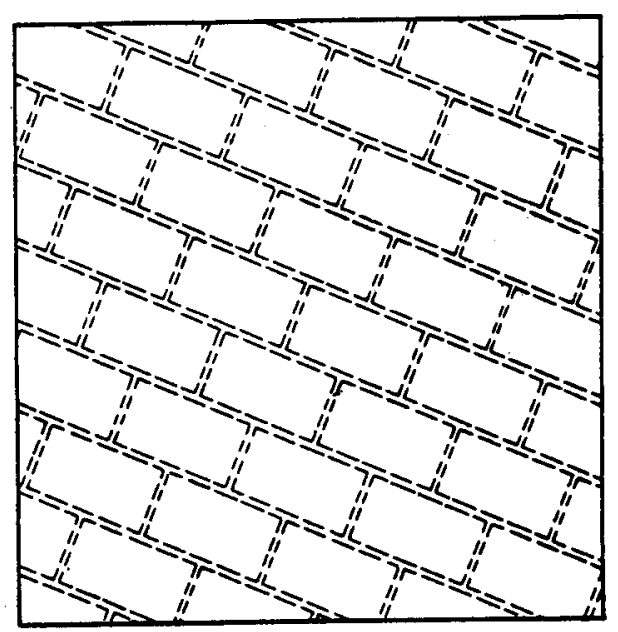

$\alpha=67.5^{\circ}$ :

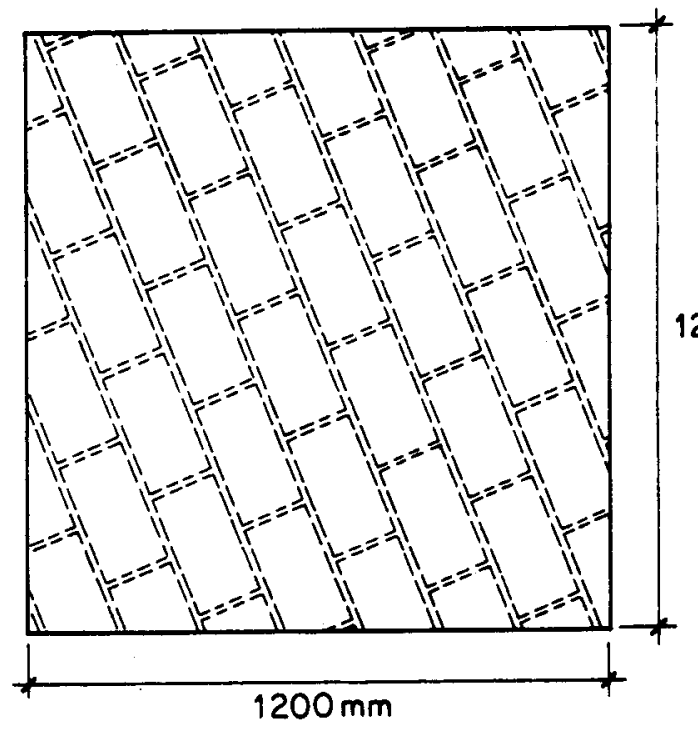

$1200 \mathrm{~mm}$

Versuchsserien : $4,5,6,10,11,12$

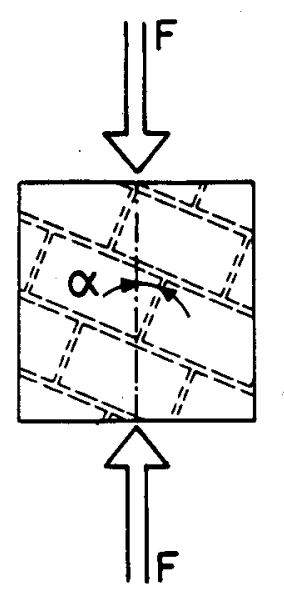

$\alpha=90^{\circ}:$

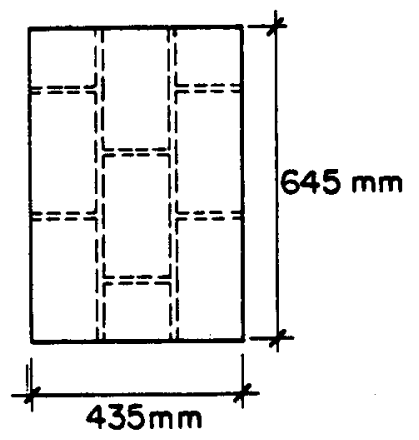


$\alpha=0^{\circ}:$

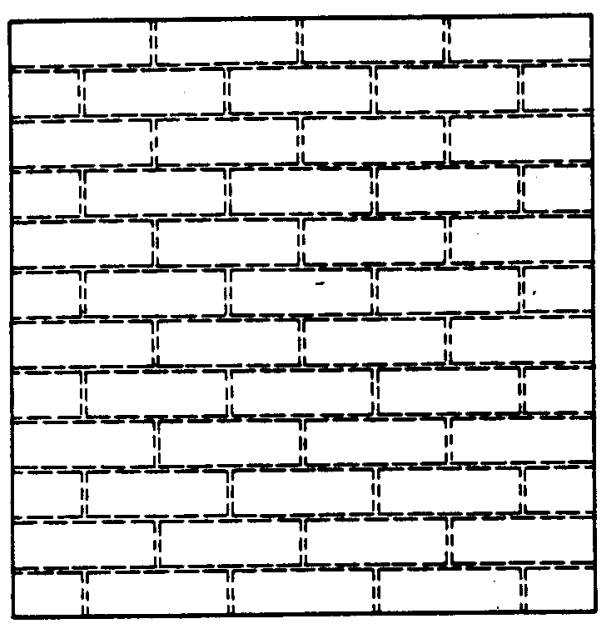

$\alpha=45^{\circ}$ :

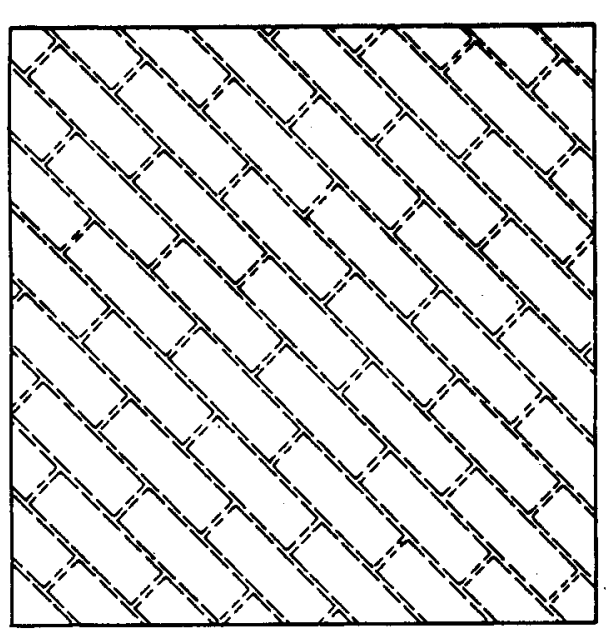

$\alpha=22.5^{\circ}$ :

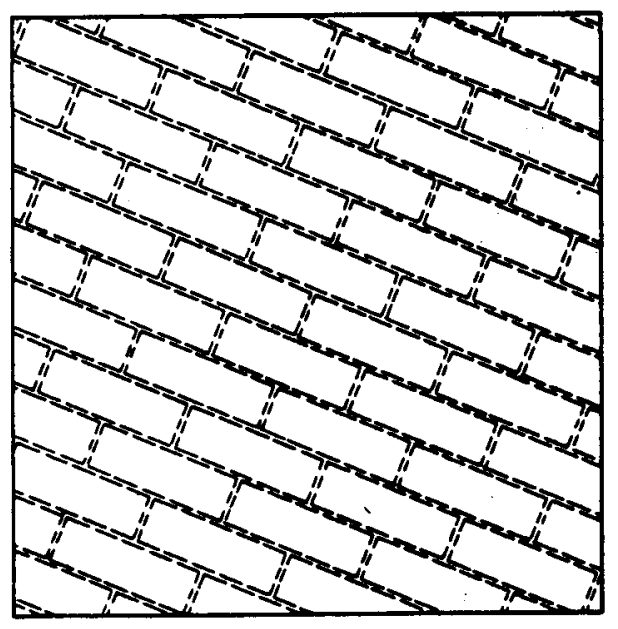

$\alpha=67.5^{\circ}$ :

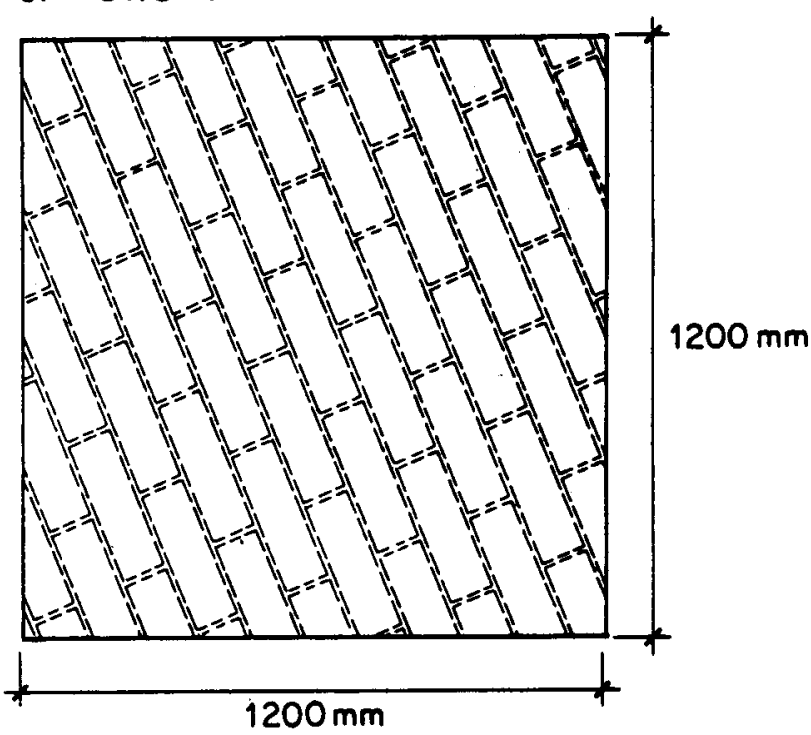

$\alpha=90^{\circ}:$

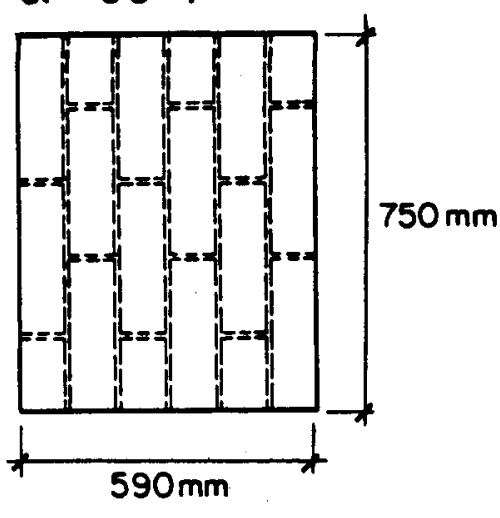

Versuchsserien : 7,8

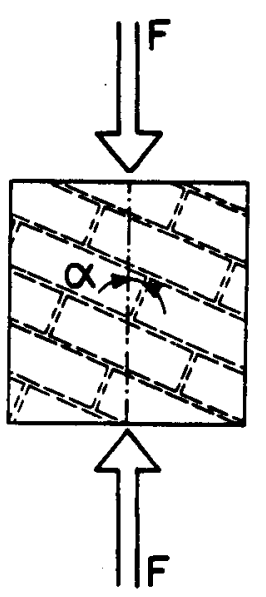

Bild 3 : Versuchskörper mit Sichtbacksteinen und Schalldämmsteinen "Calmo" 
a) Pressfuge mit verdecktem Spatz:

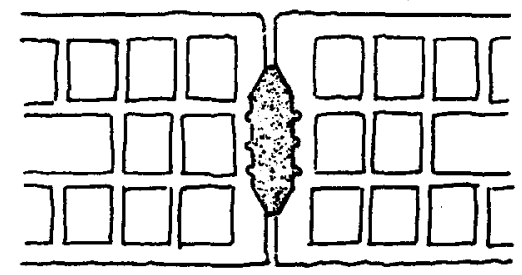

c) Voll vermörtelte Fuge : b) Halb vermörtelte Fuge:

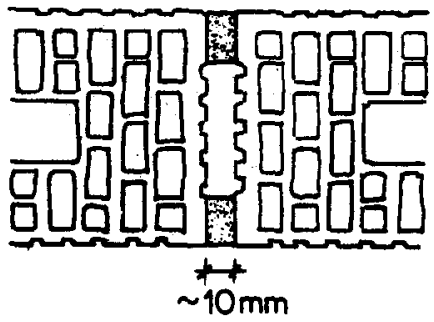

Bild 4: Ausbildung der Stossfugen
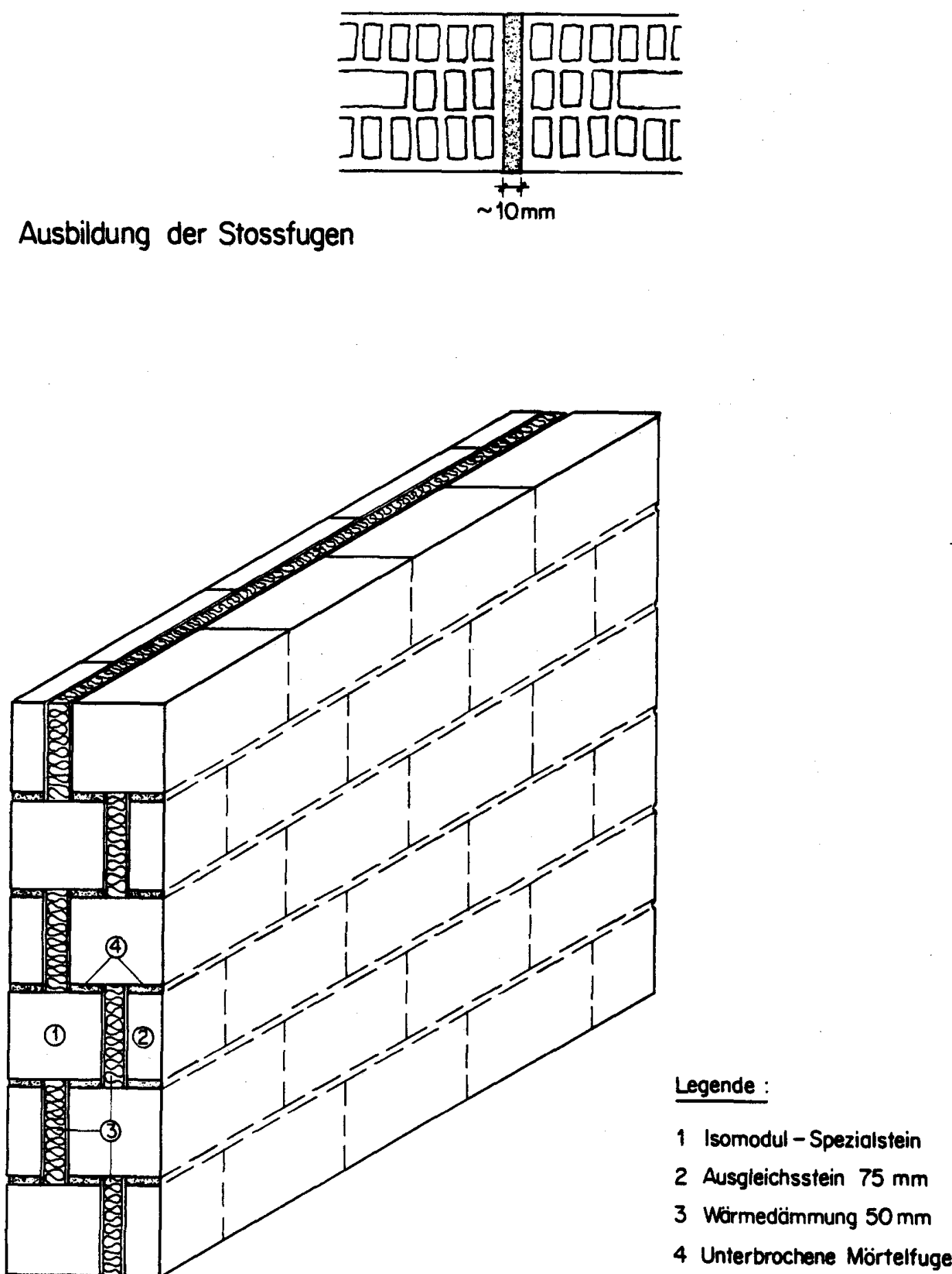

Bild 5: Isomodul-Mauerwerk ( $0^{\circ}$-Körper ) 

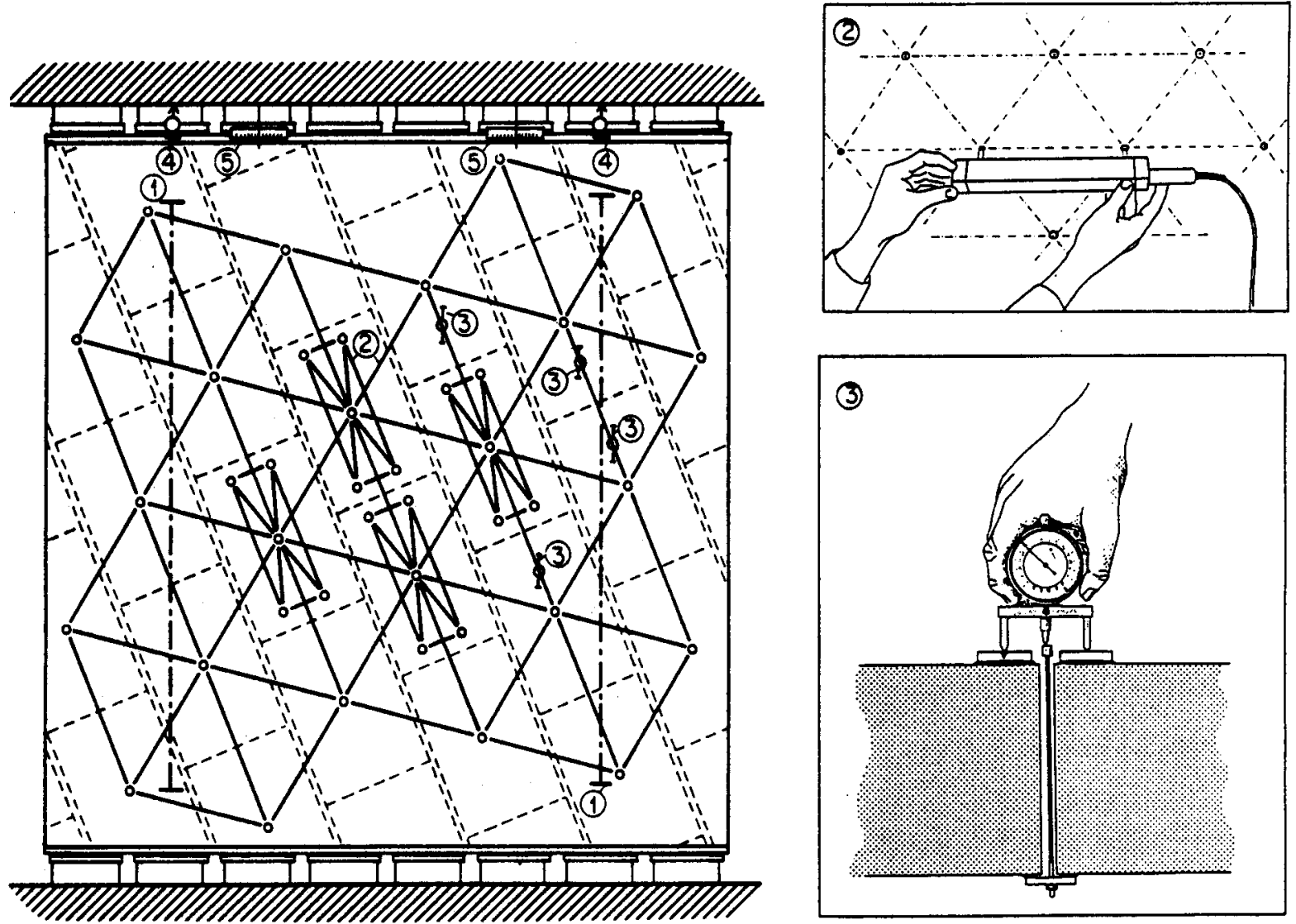

(1) Induktive Weggeber (Versuchssteuerung)

(2) Deformeter (Messnetze) : A) Messung der Verformung des Versuchskörpers

B) Messung der Verformung der Steine

(3) Messung der Verändenung der Wandstärke $\$$

(5) Verformung der Elastomer-Lager

$\left\{\begin{array}{l}\text { Vertikale Richtung (Messuhren) } \\ \text { Schiebungen }\end{array}\right.$

TIPl, Stahiplatten der Presse

Bild 6 : Anordnung der Messungen am Beispiel eines Versuchskörpers mit $\alpha=67.5^{\circ}$ Fugenneigung 

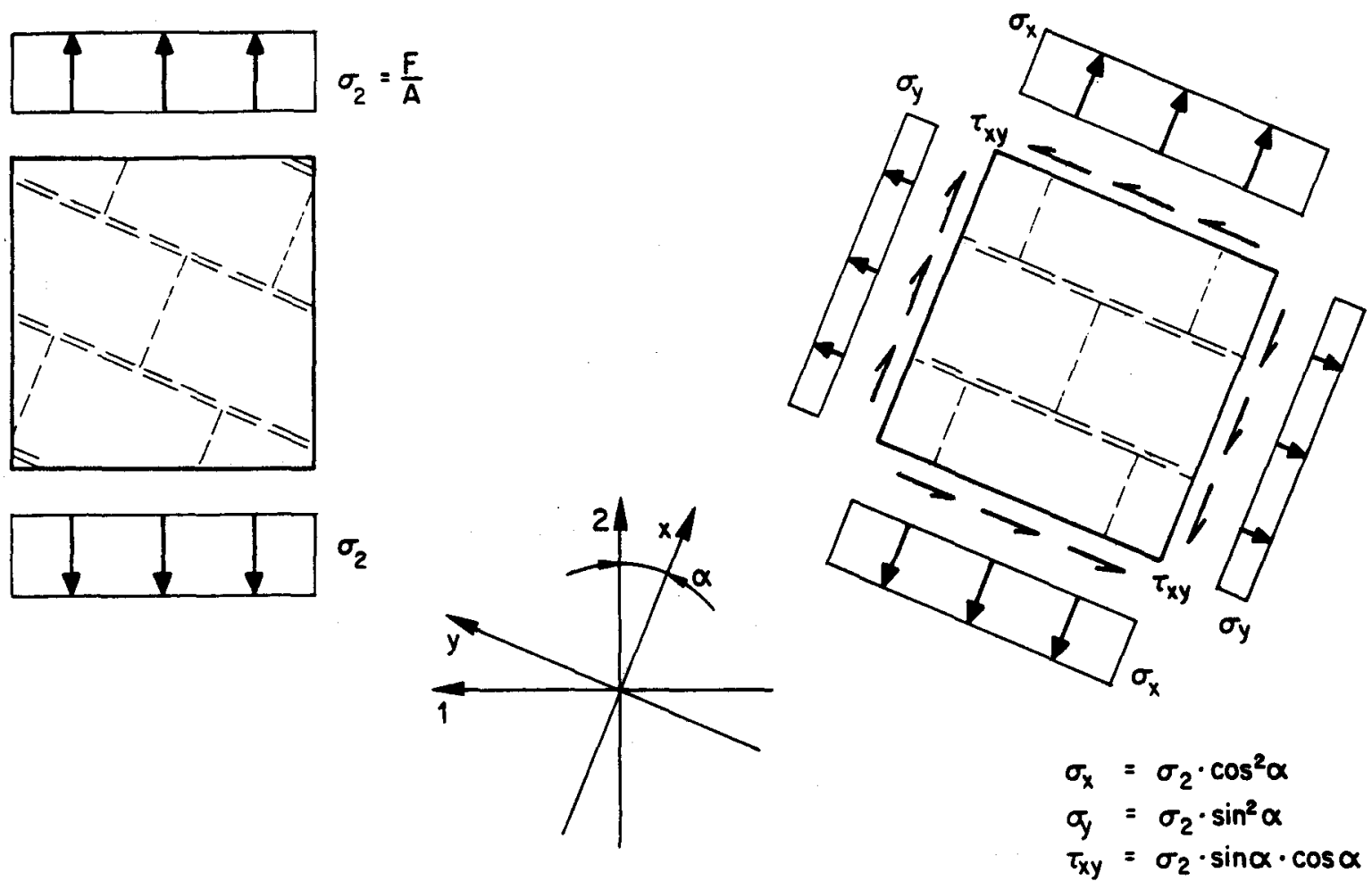

Bild 7: Spannungstransformation

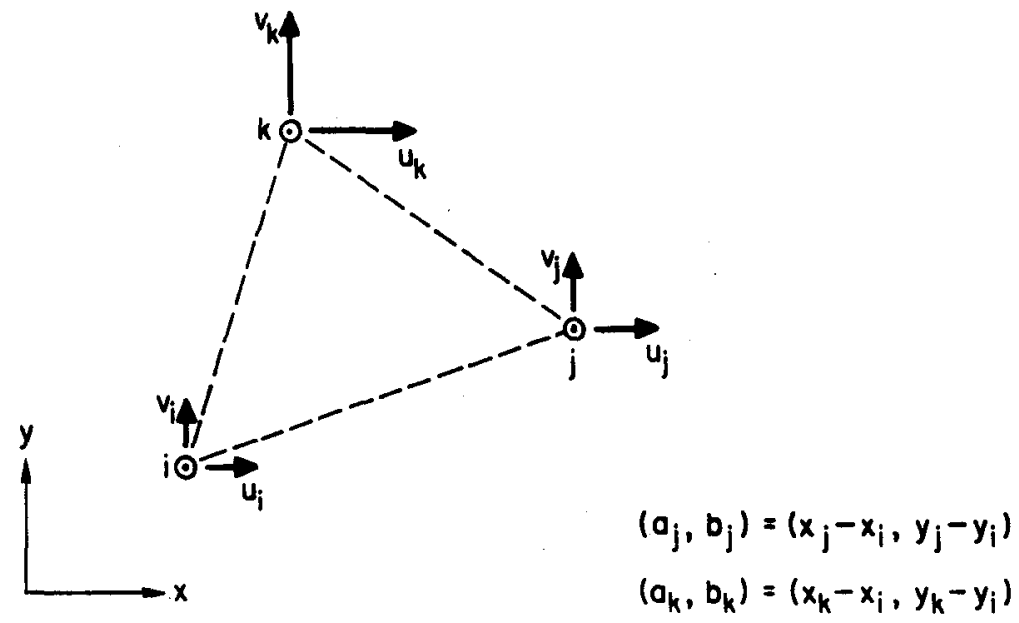

$$
\begin{aligned}
& \{\epsilon\}=[B] \cdot\{\delta\} \\
& \left\{\begin{array}{l}
\epsilon_{x} \\
\epsilon_{y} \\
\gamma_{x y}
\end{array}\right\}=\left[\begin{array}{cccccc}
\left(b_{j}-b_{k}\right) & 0 & b_{k} & 0 & -b_{j} & 0 \\
0 & \left(a_{k}-a_{j}\right) & 0 & -a_{k} & 0 & a_{j} \\
\left(a_{k}-a_{j}\right) & \left(b_{j}-b_{k}\right) & -a_{k} & b_{k} & a_{j} & -b_{j}
\end{array}\right] \cdot\left\{\begin{array}{l}
u_{j} \\
v_{j} \\
u_{j} \\
v_{j} \\
u_{k} \\
v_{k}
\end{array}\right\}
\end{aligned}
$$

Bild 8: Berechnung der Verzerrungen aus den Knotenverschiebungen des Messnetzes 

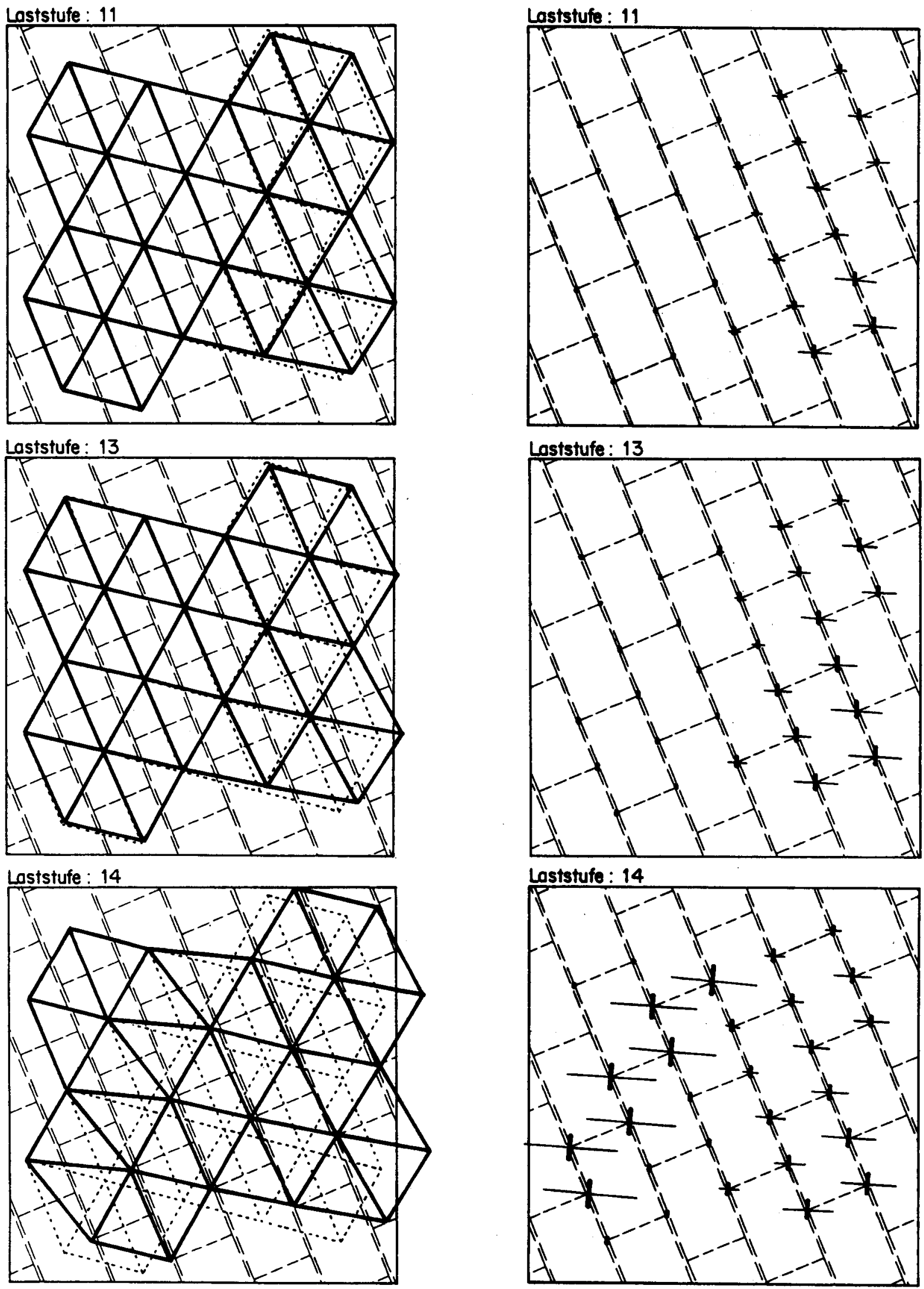

Knoterverschiebungen :

Verschiebungsmassstob: $\longmapsto 10.00[\mathrm{~mm}]$

Loststufe: 13

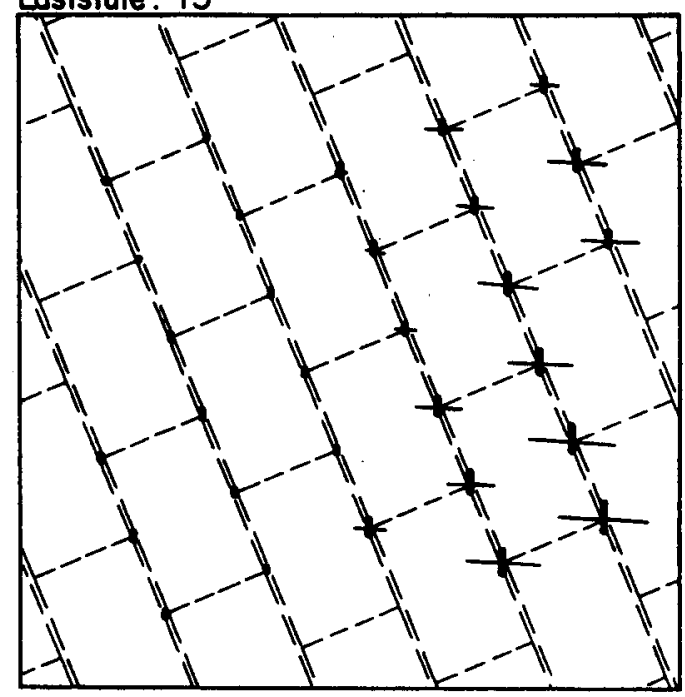

Loststufe: 14

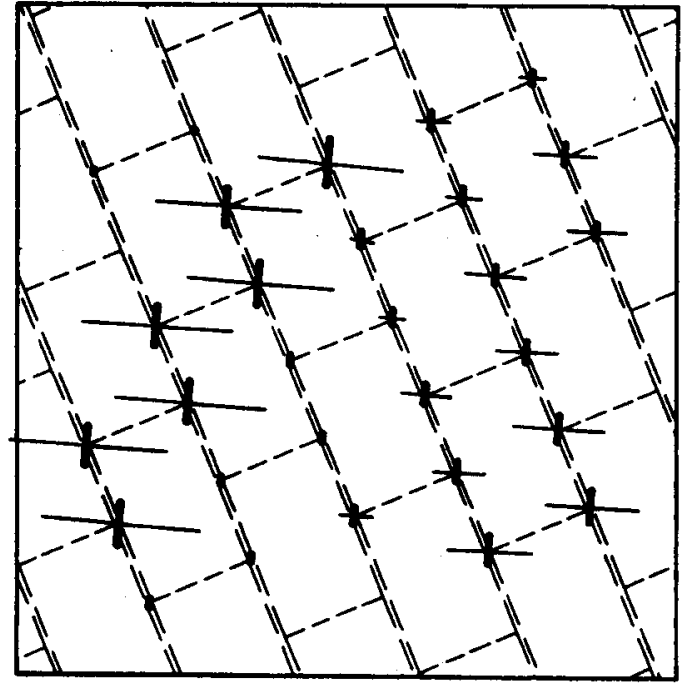

Hauptdehnungen :

Dehnungsmassstab : $\longmapsto 0.01$

Bild 9: Versuch $\mathrm{K} 63$ 
Laststufe: 11
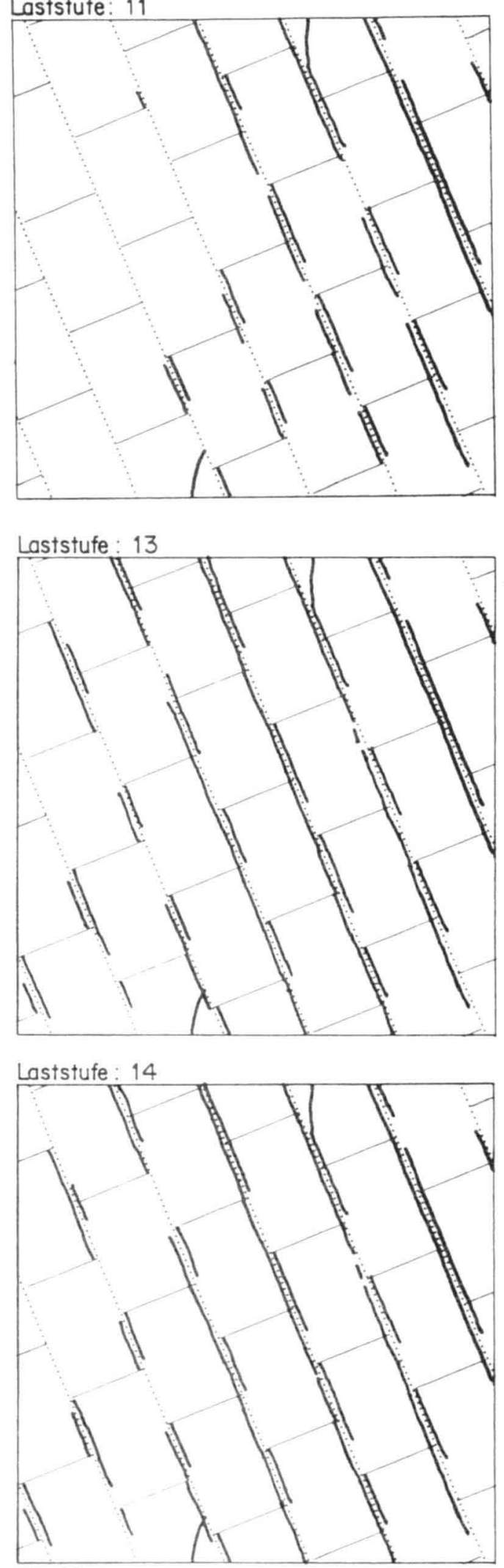

Rissbilder
Bereich mit kleinen Rissen
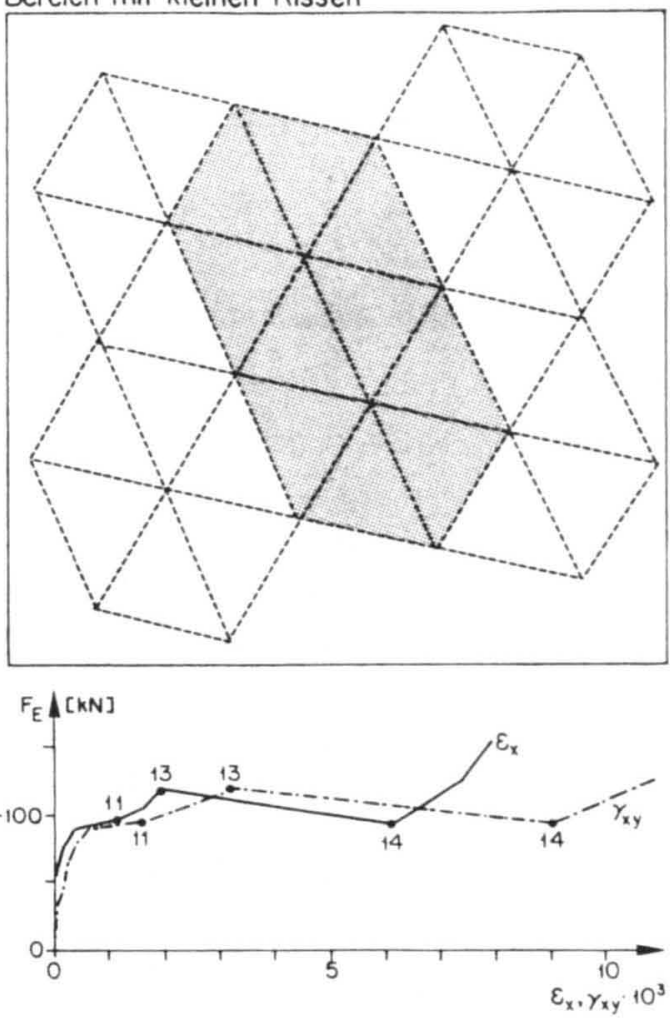

Bereich mit klafendem Riss
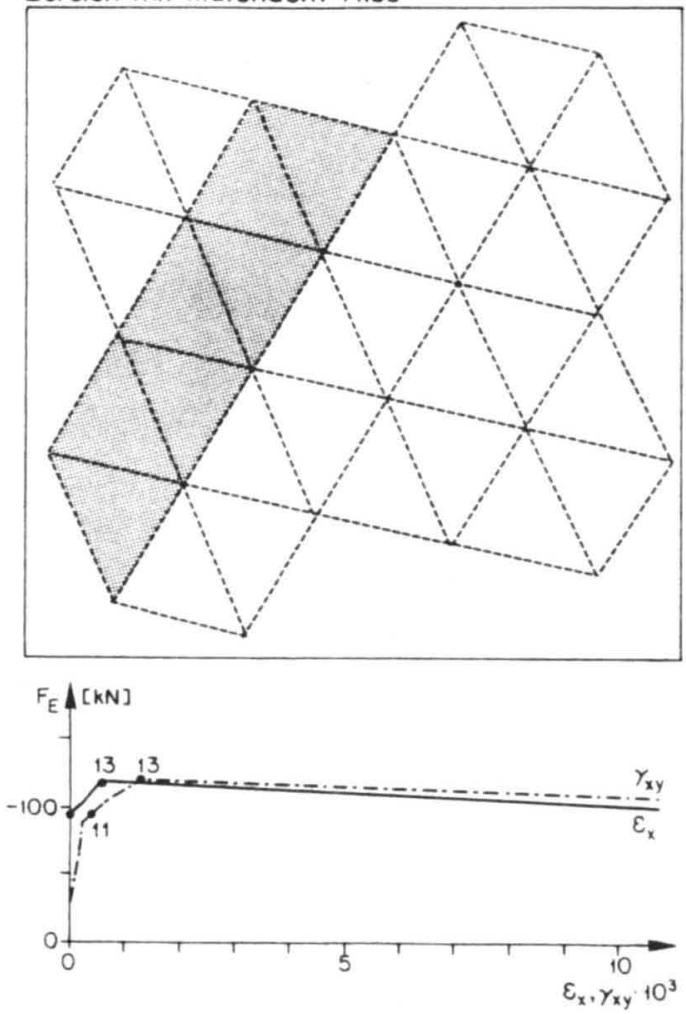

Abhängigkeit der Verzerrungen vom Bereich der Mittelbildung : 


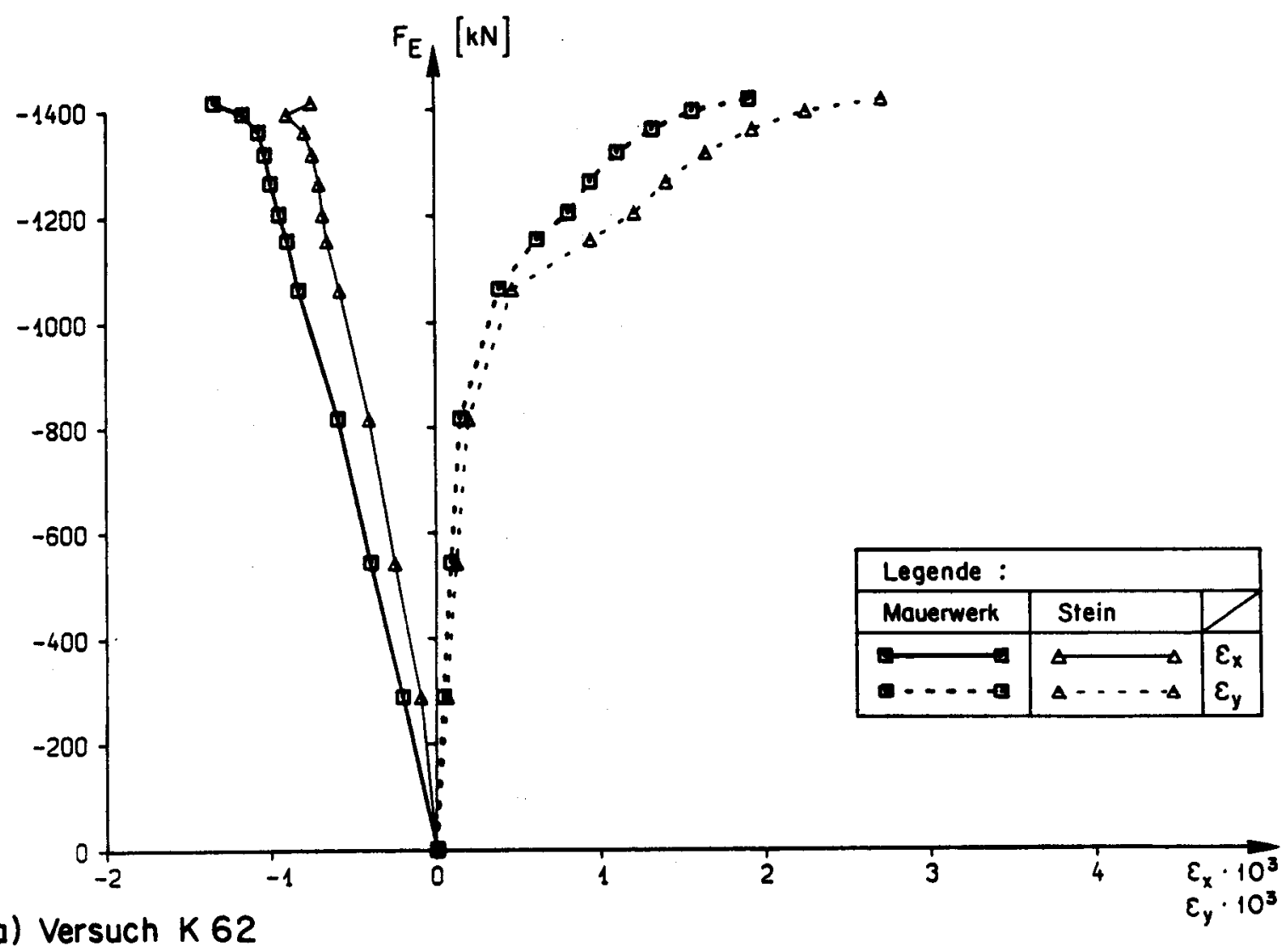

a) Versuch $K 62$

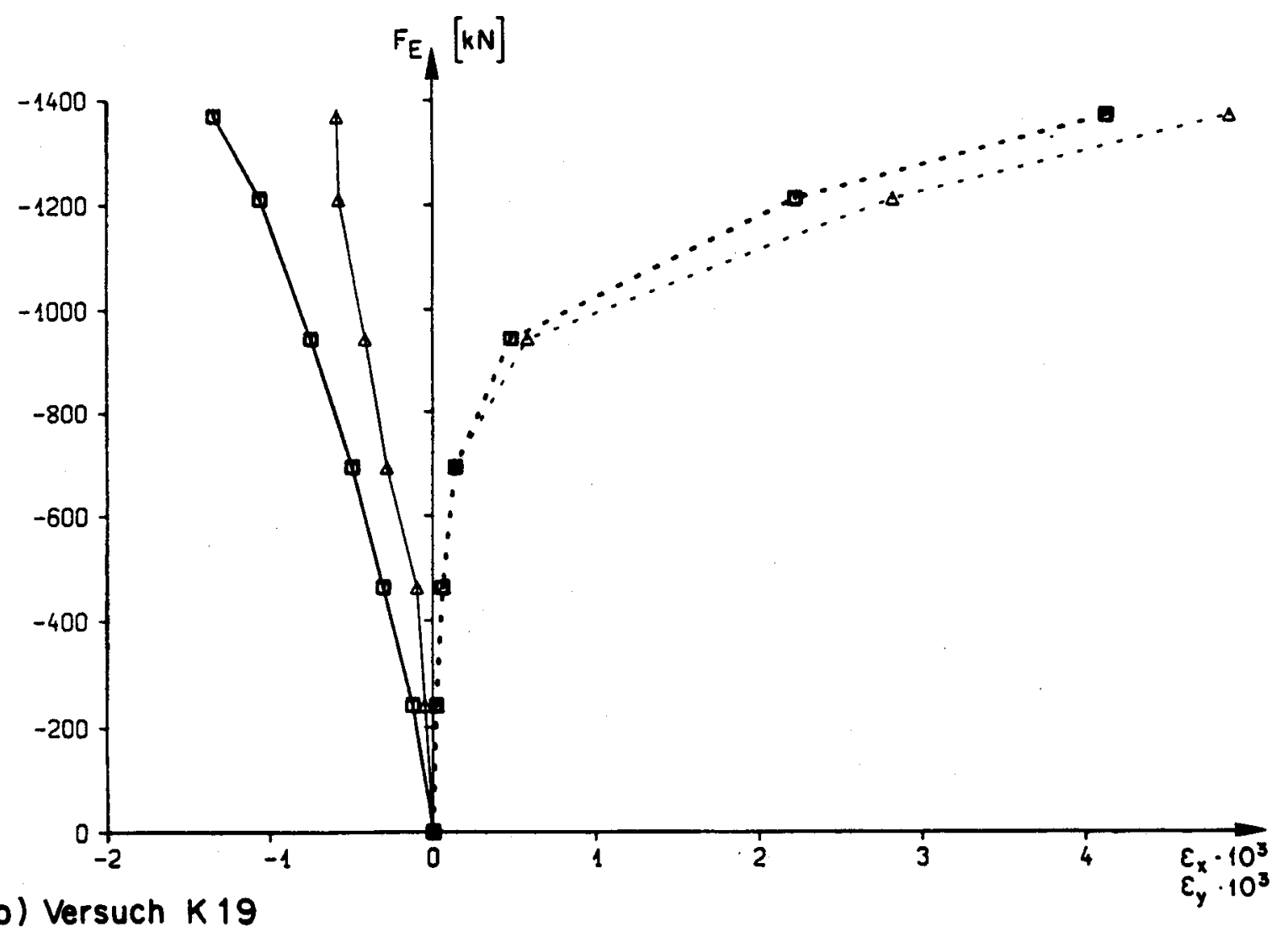

Bild 10: Lost-Verzerrungsdiagramme, Versuche mit $0^{\circ}$ Fugenneigung 

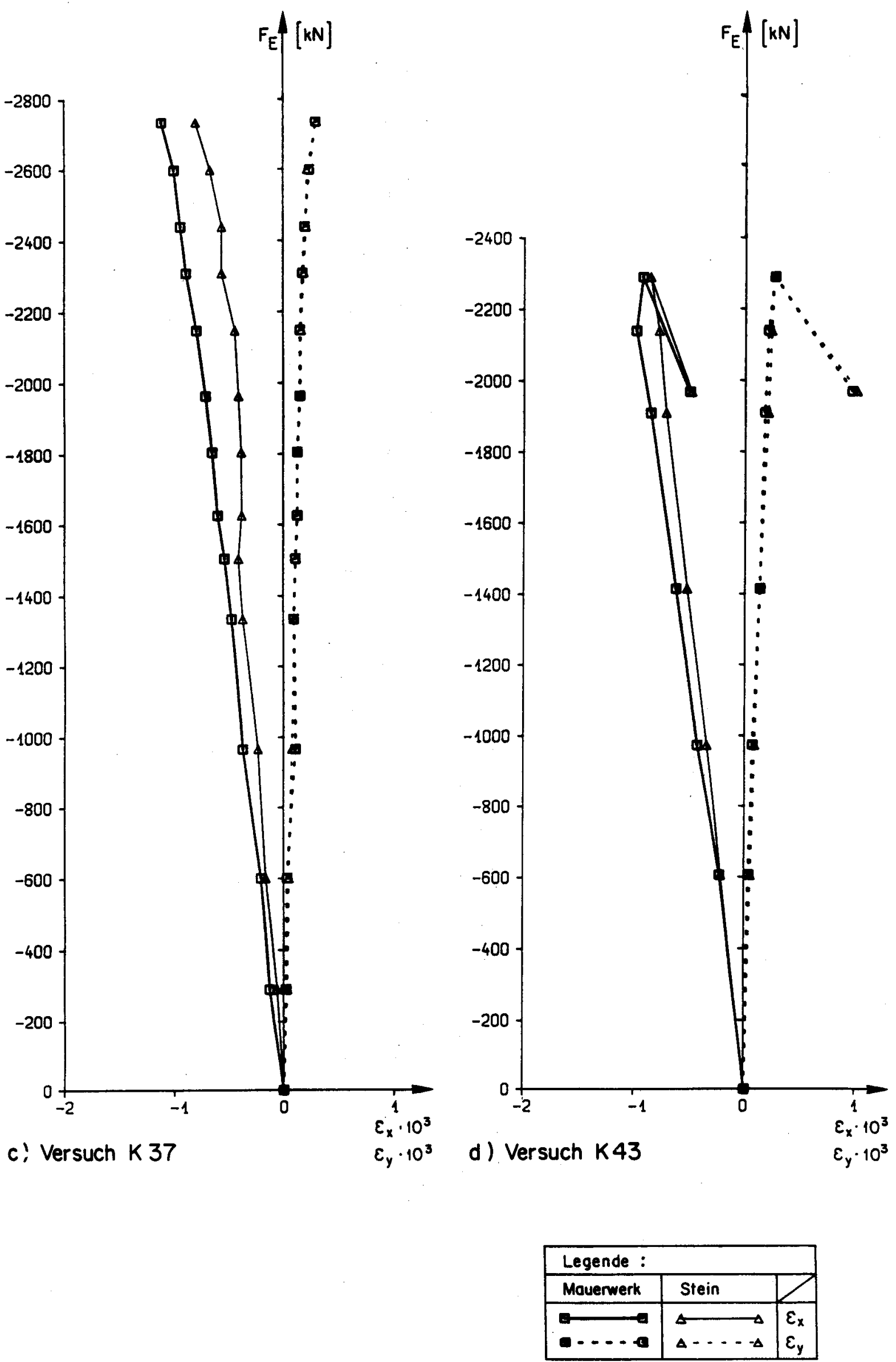

Bild 10: Fortsetzung; Versuche mit $0^{\circ}$ Fugenneigung 

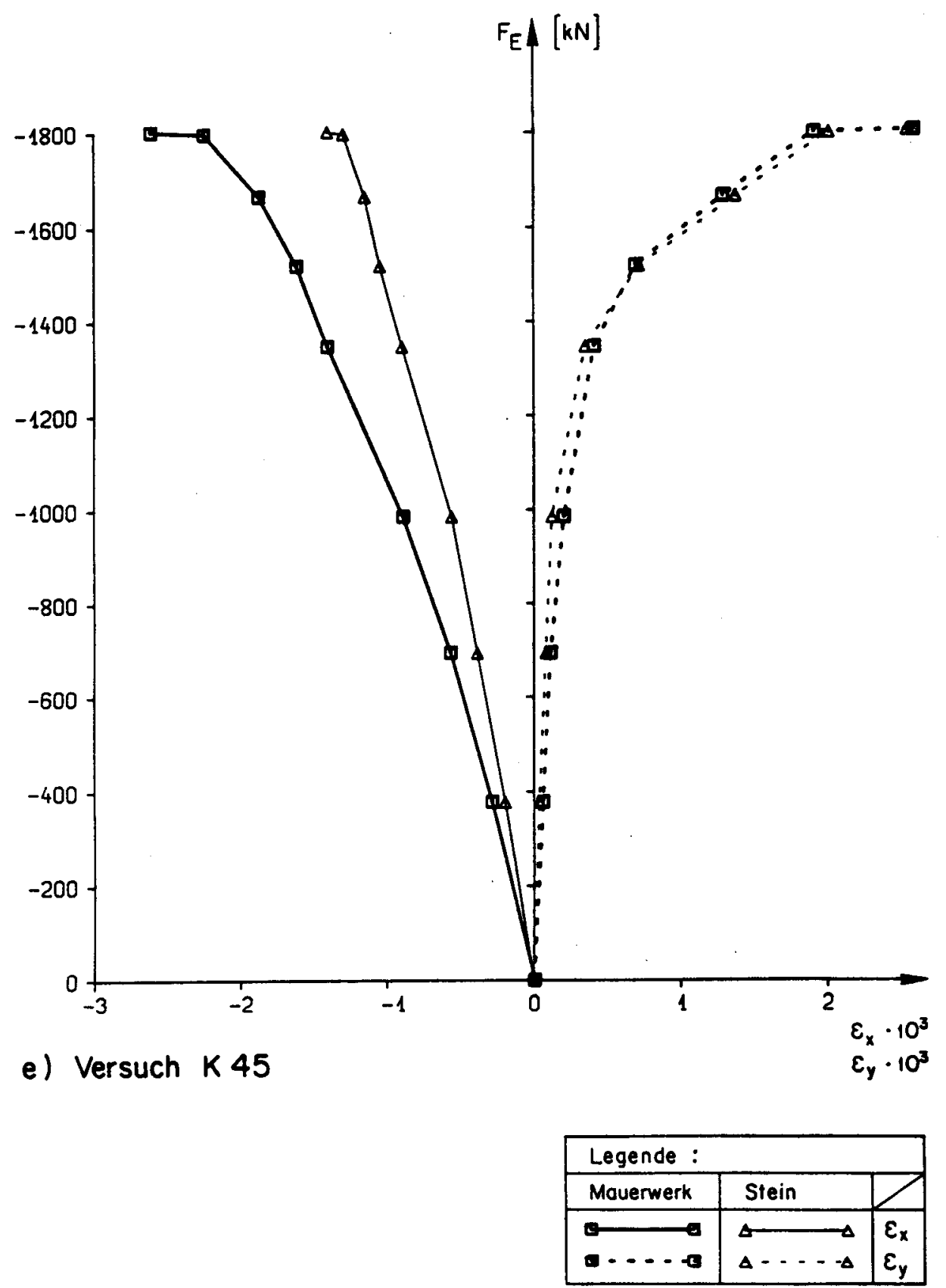

Bild 10: Fortsetzung; Versuche mit $0^{\circ}$ Fugenneigung 

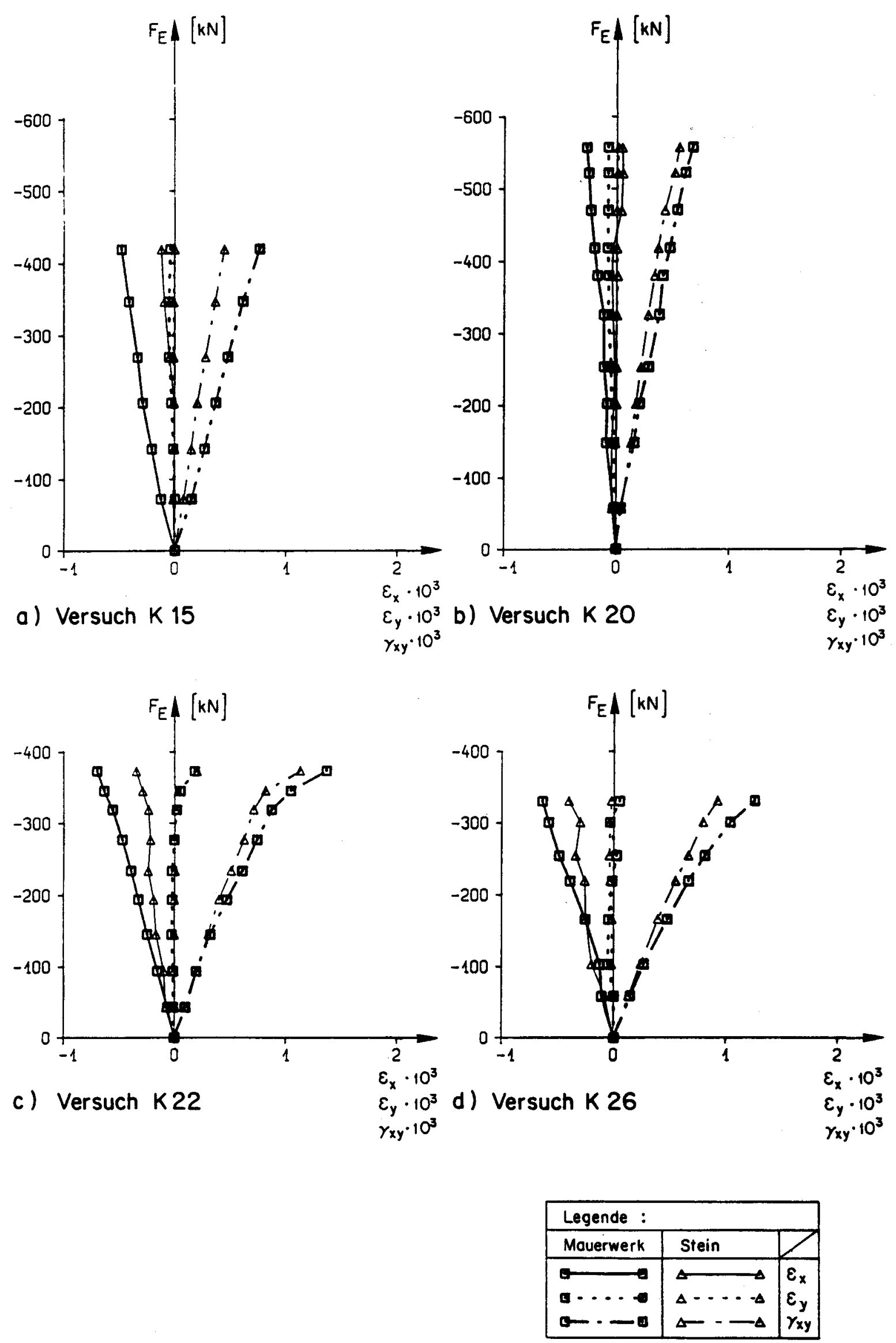

Bild 11: Last-Verzerrungsdiagramme; Versuche mit $22.5^{\circ}$ Fugenneigung 


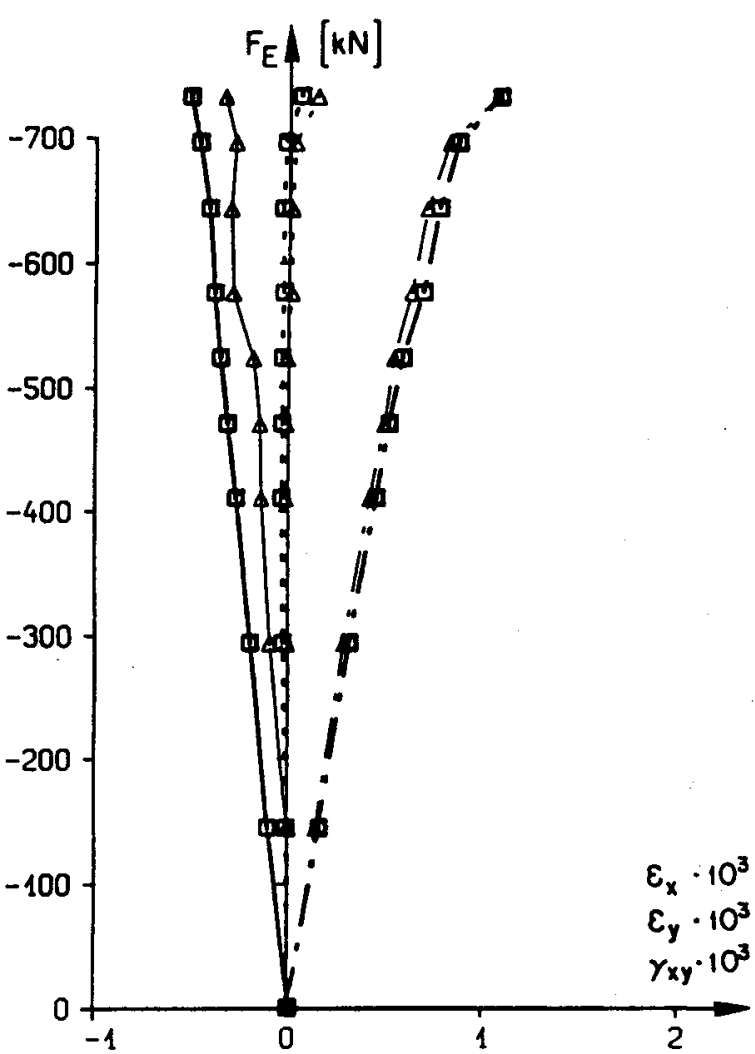

e) Versuch $\mathrm{k} 30$

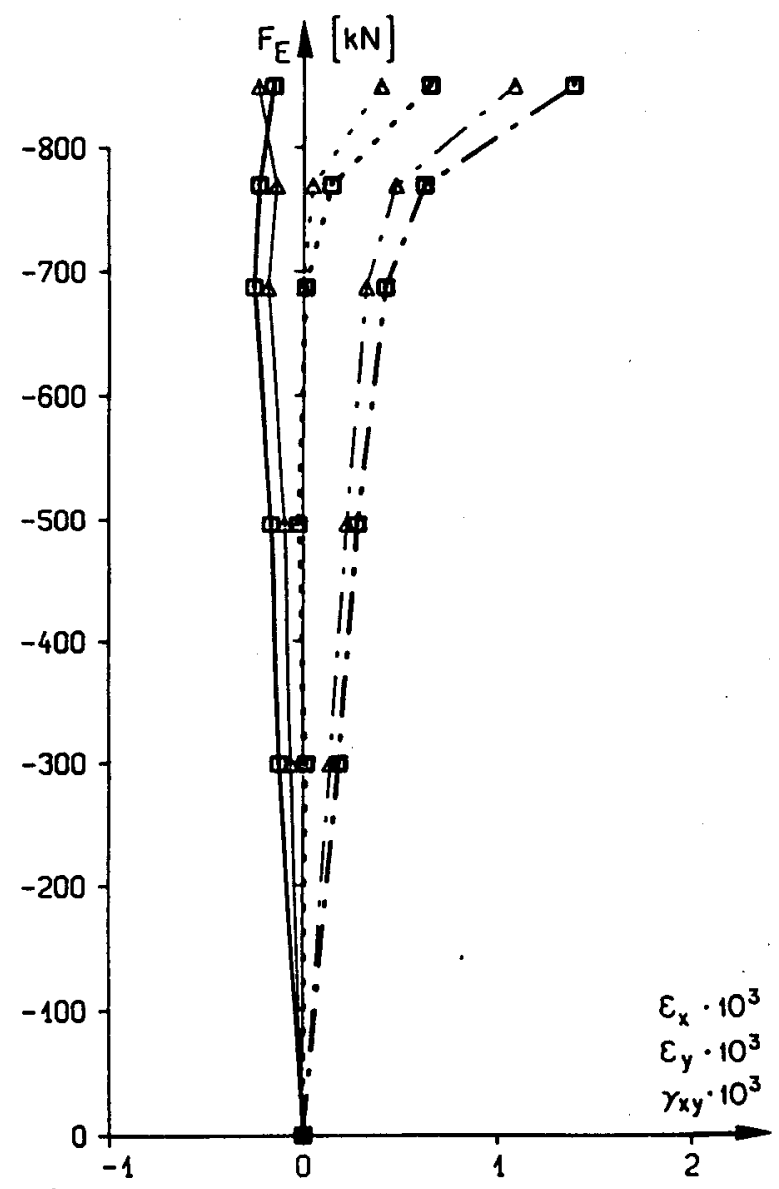

g) Versuch K 44

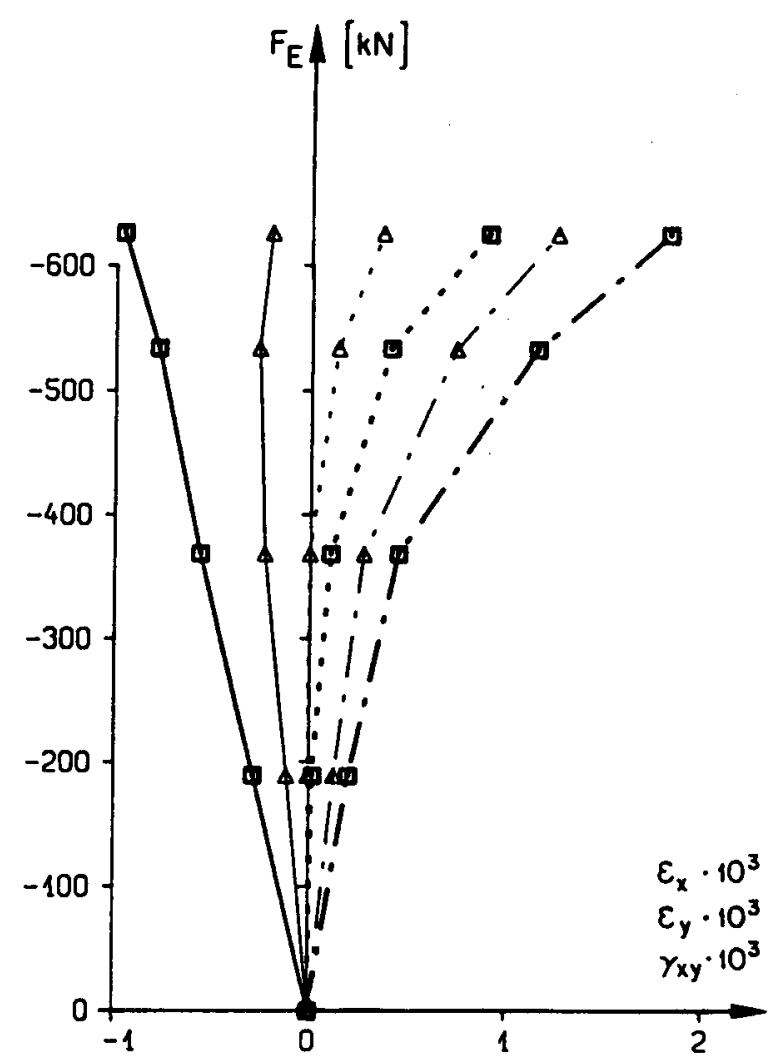

f) Versuch $\mathrm{K} 34$

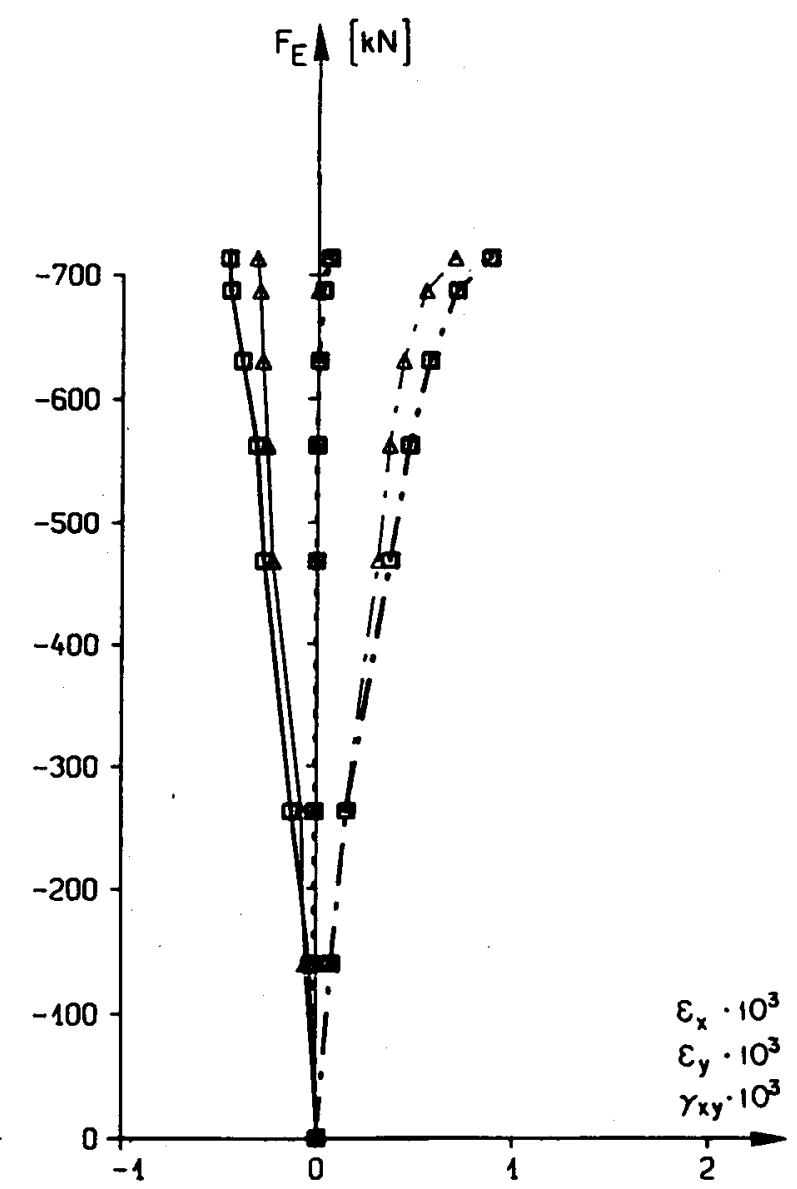

h) Versuch K 46

Bild 11: Fortsetzung; Versuche mit $22.5^{\circ}$ Fugenneigung 

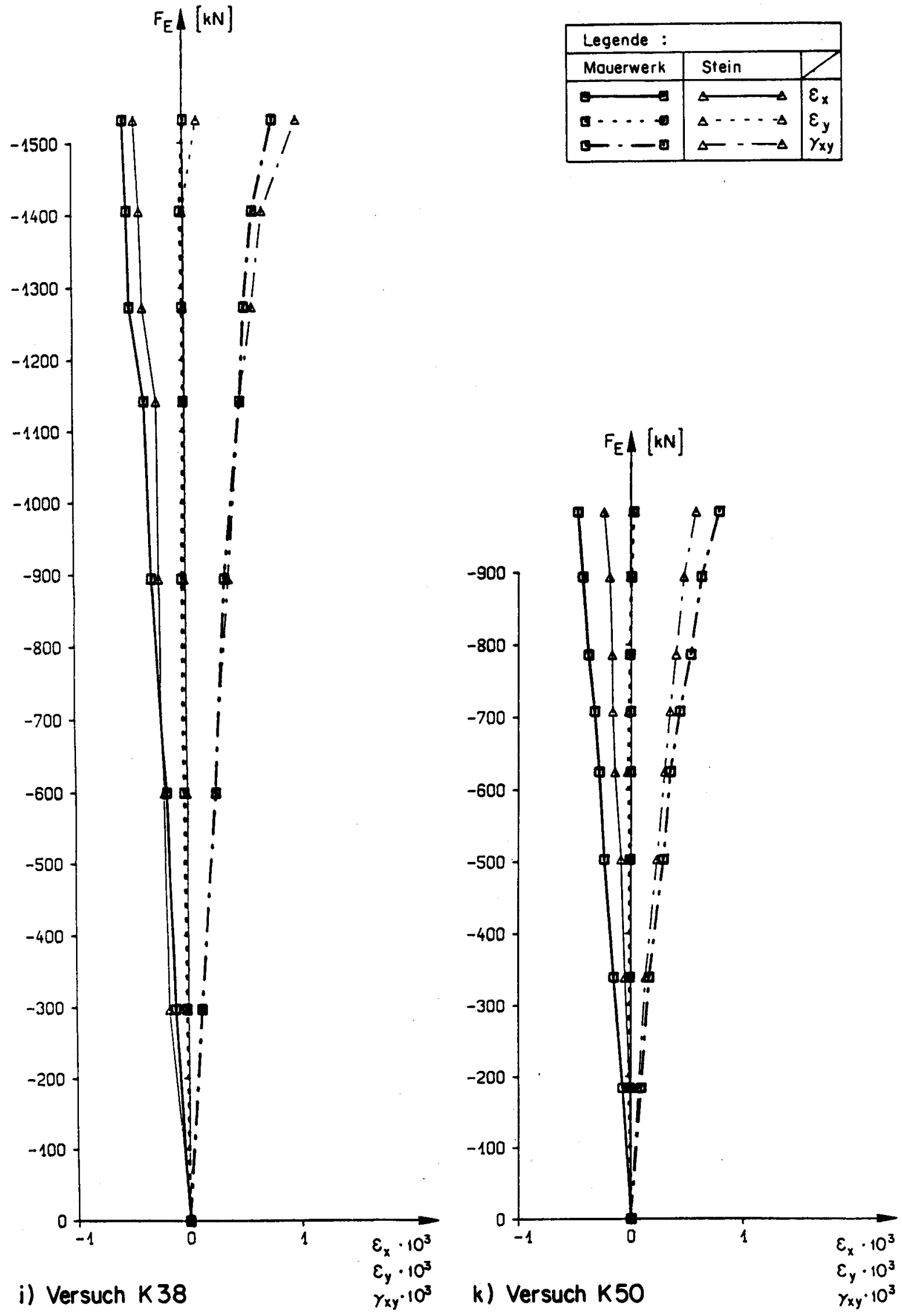

Bild 11: Fortsetzung; Versuche mit $22.5^{\circ}$ Fugenneigung 

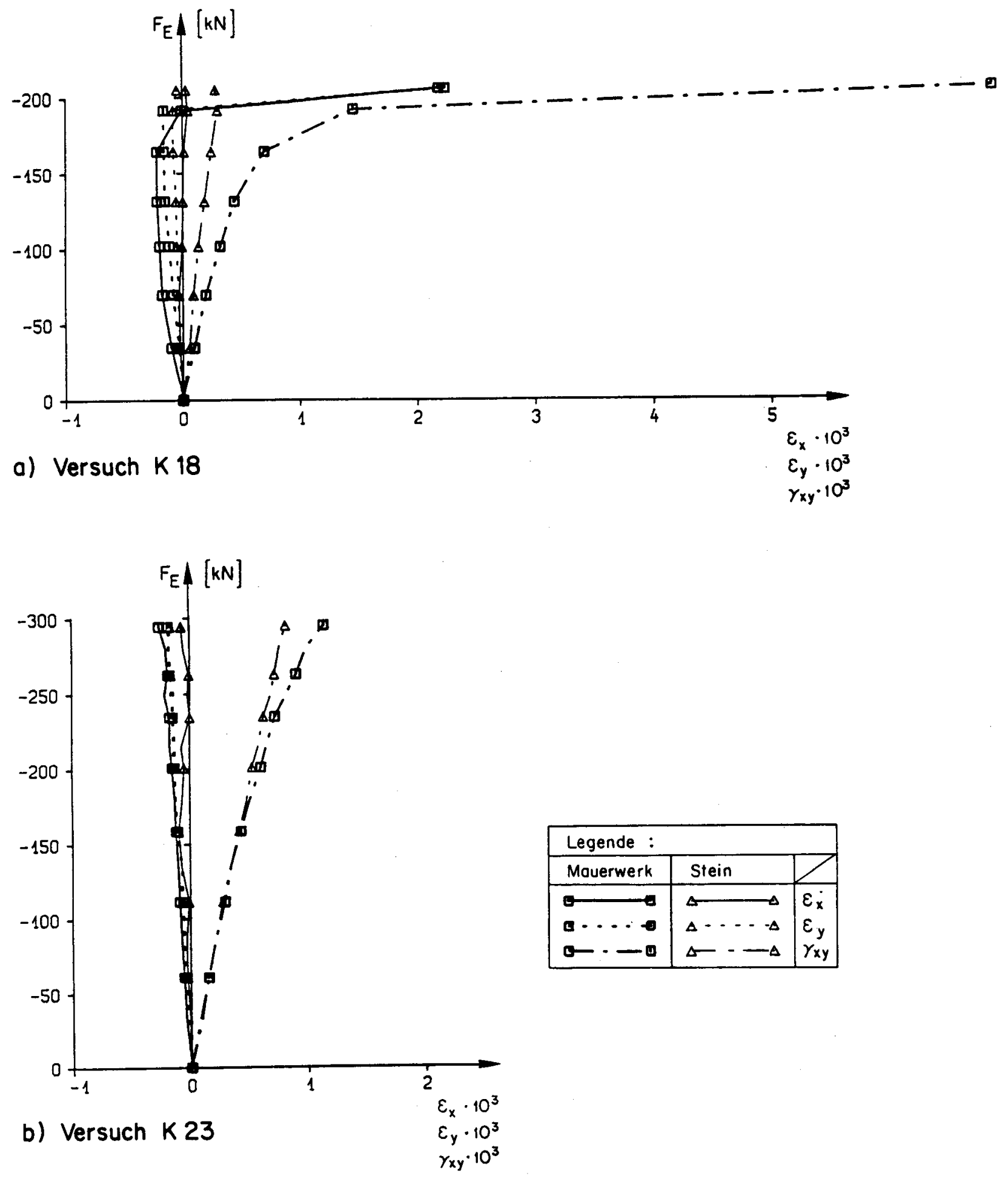

Bild 12: Last-Verzerrungsdiagramme; Versuche mit $45^{\circ}$ Fugenneigung 

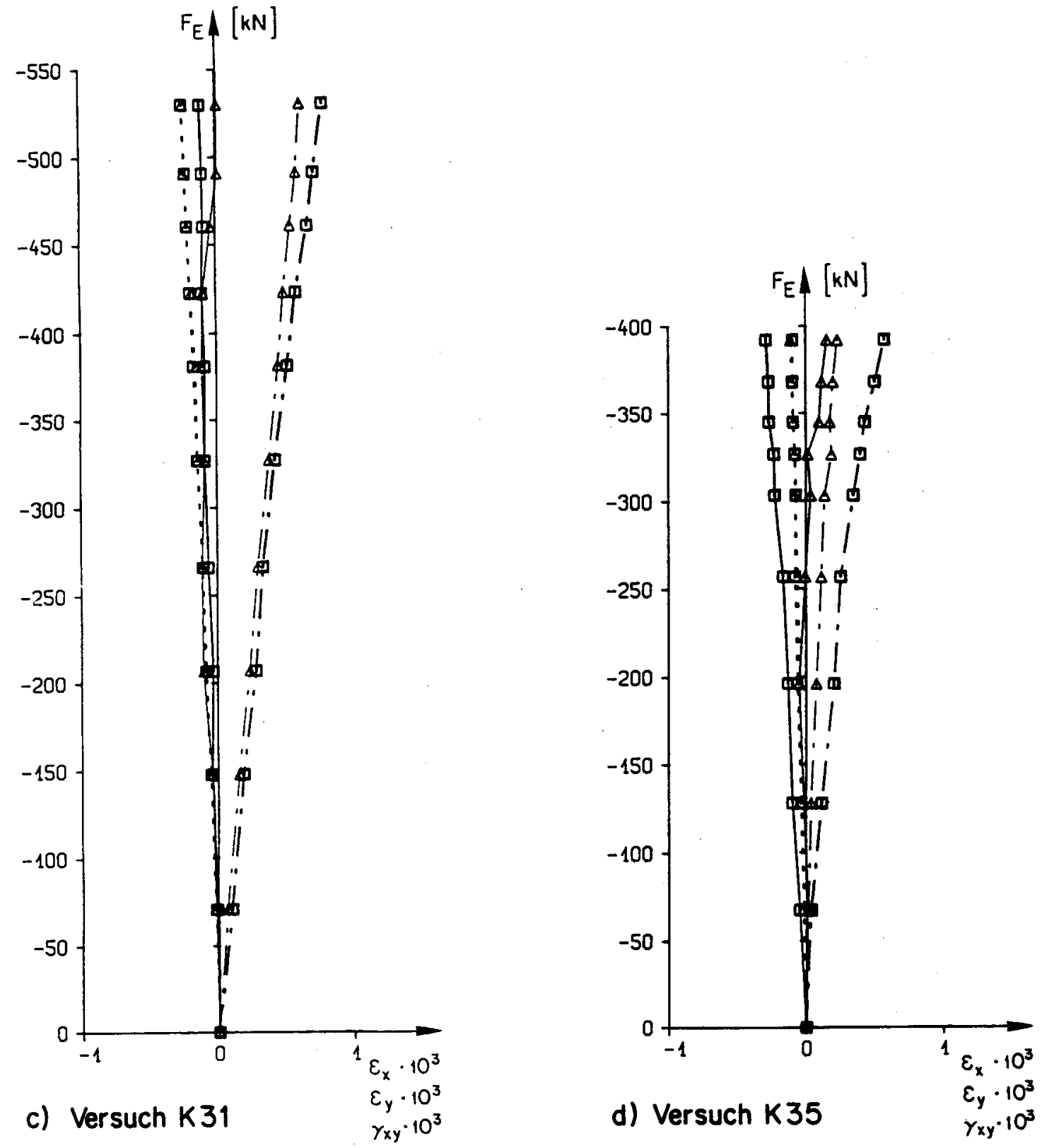

\begin{tabular}{|l|l|l|}
\hline \multicolumn{2}{|l|}{ Legende : } \\
\hline Mouerwerk & Stein & \\
\hline$\Delta-\Delta$ & $\Delta-\Delta$ & $\varepsilon_{x}$ \\
$\Delta \ldots .-\Delta$ & $\varepsilon_{y}$ \\
$\Delta-.-\Delta$ & $\Delta-\Delta$ & $\gamma_{x y}$ \\
\hline
\end{tabular}

Bild 12: Fortsetzung; Versuche mit $45^{\circ}$ Fugenneigung 

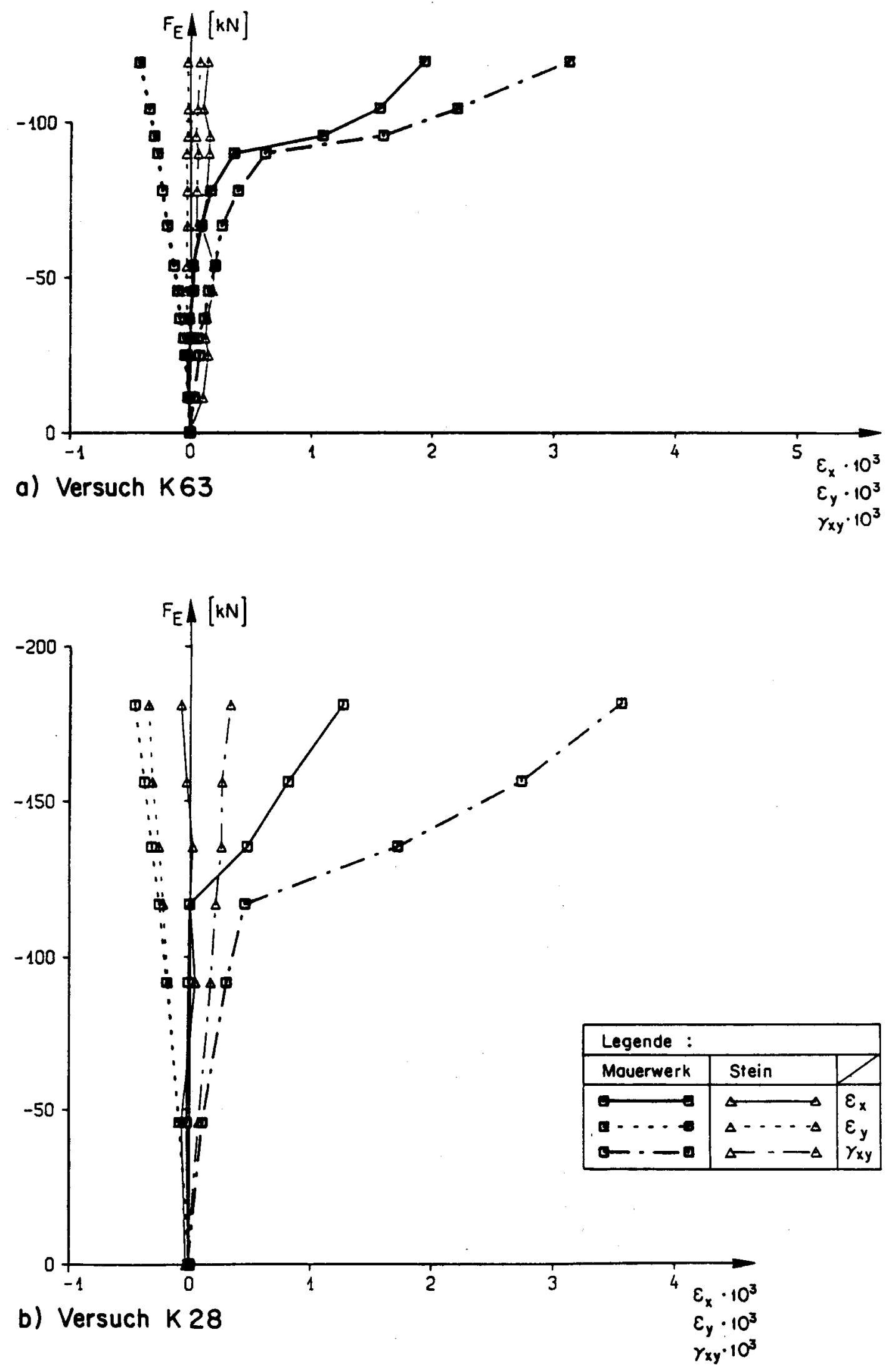

Bild 13: Last-Verzerrungsdiagramme; Versuche mit $67.5^{\circ}$ Fugenneigung 


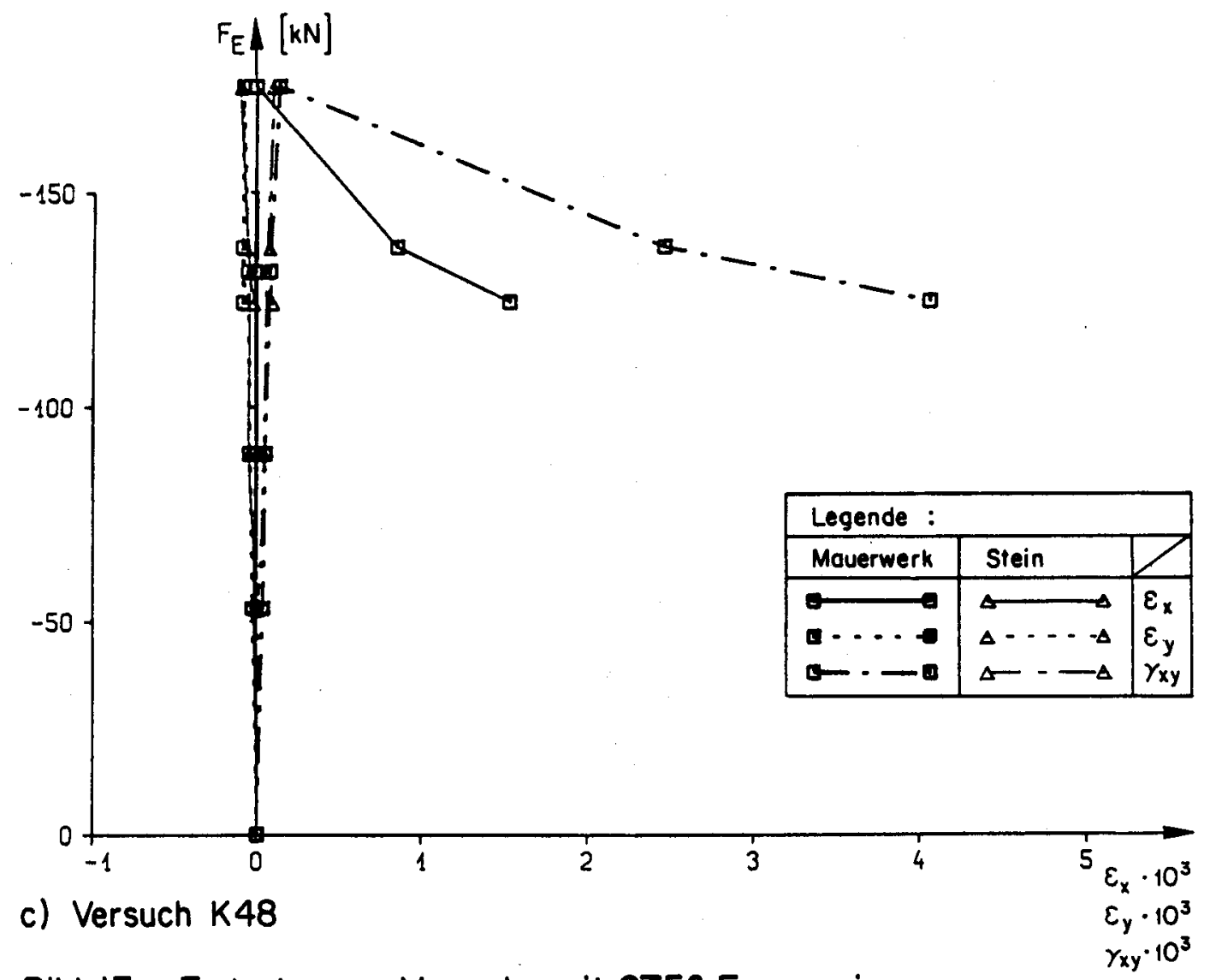

Bild 13: Fortsetzung; Versuche mit $67.5^{\circ}$ Fugenneigung

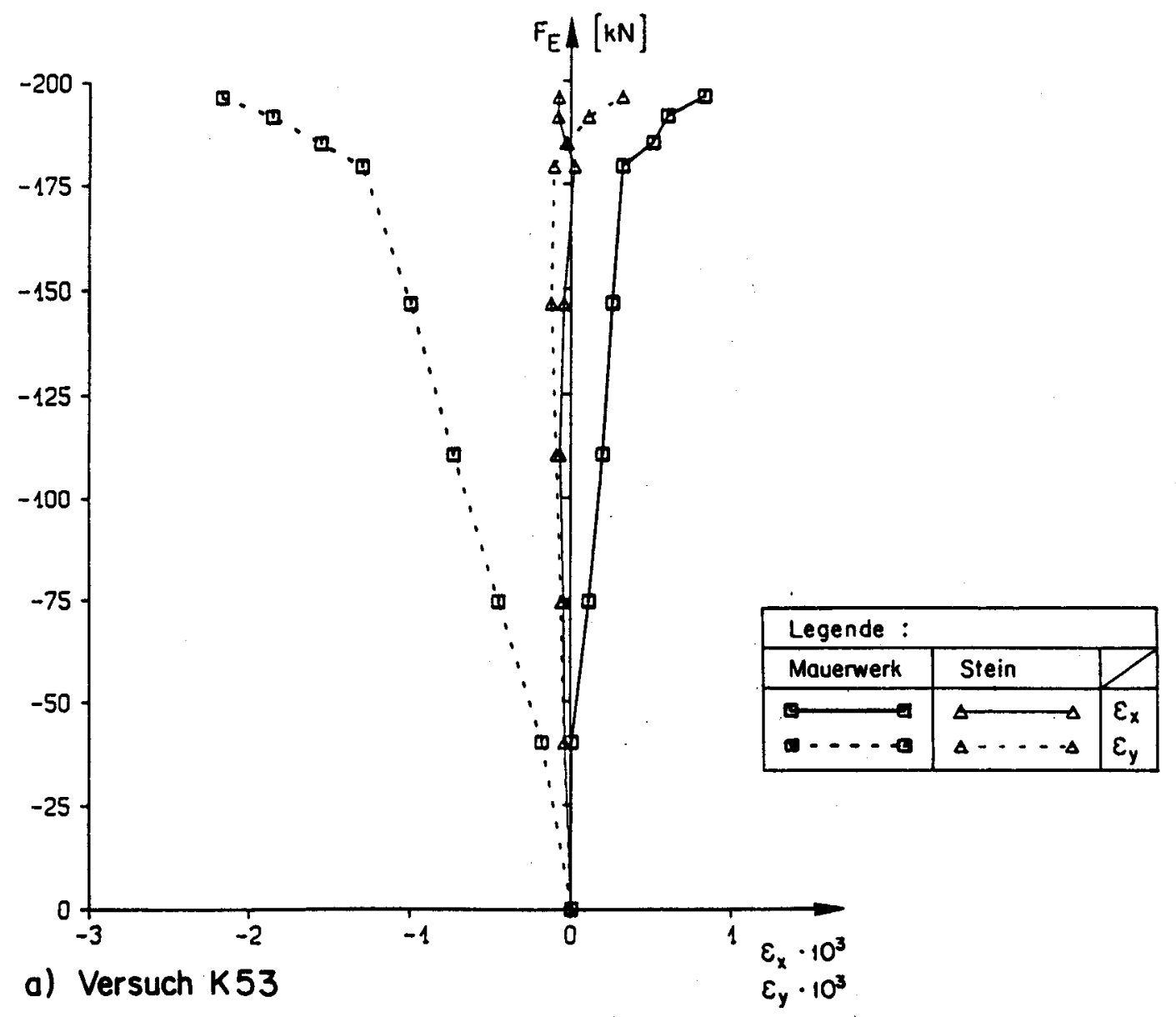

Bild 14 : Last-Verzerrungsdiagramme; Versuche mit $90^{\circ}$ Fugenneigung 

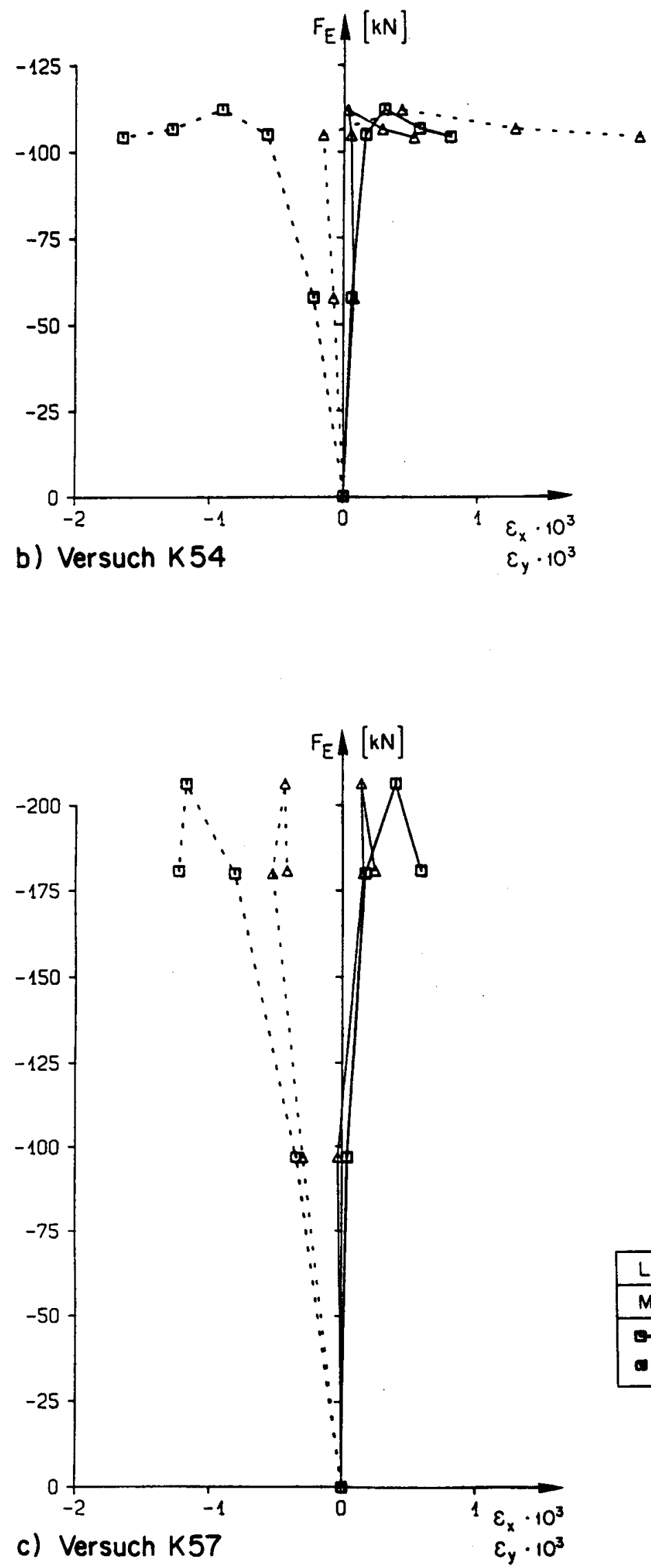

Bild 14: Fortsetzung; Versuche mit $90^{\circ}$ Fugenneigung 


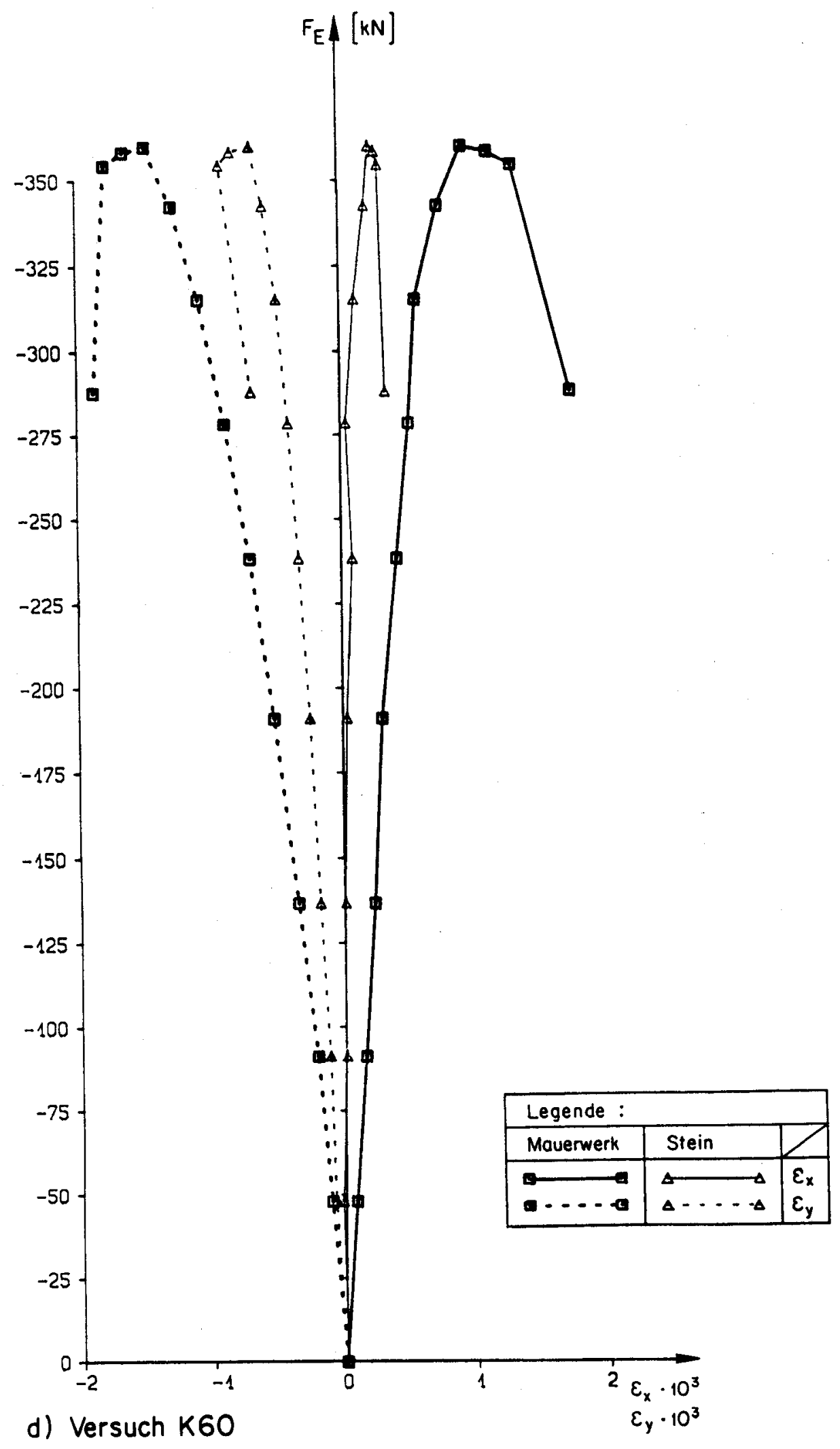

Bild 14: Fortsetzung ; Versuche mit $90^{\circ}$ Fugenneigung 
Versuch $\mathrm{K} 62$

Loststufe 12

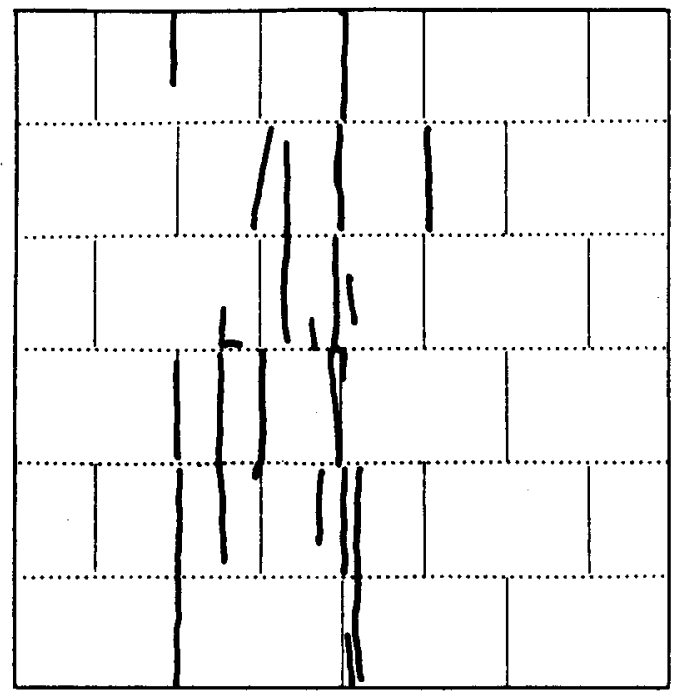

Versuch $\mathrm{K} 17$

Loststufe 10

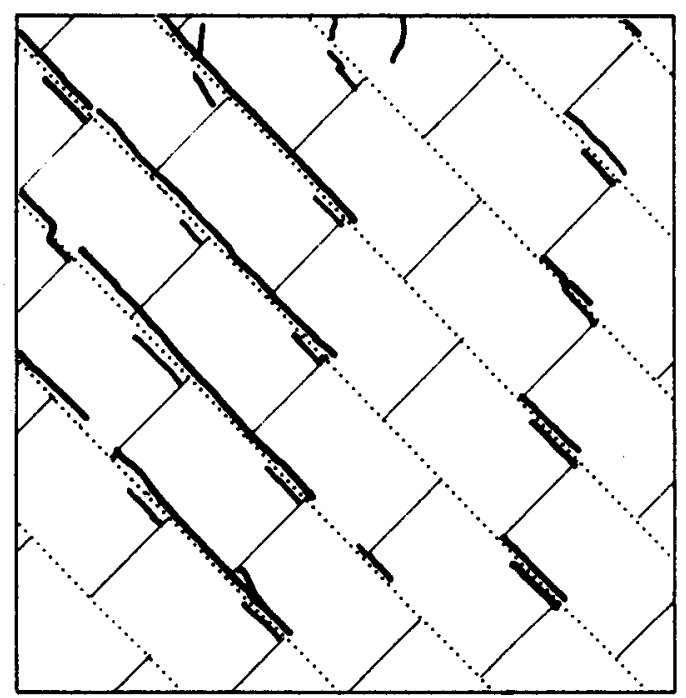

Versuch $\mathrm{K} 13$

Laststufe 13

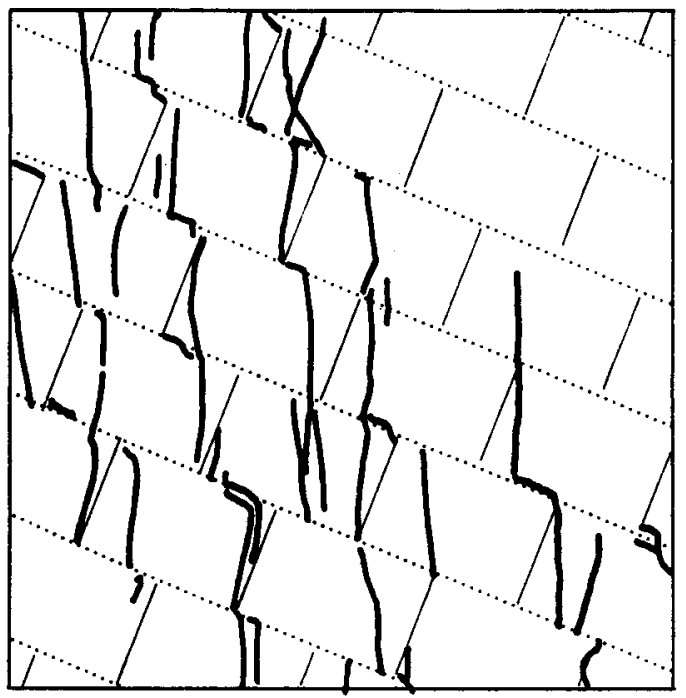

Versuch $\mathrm{K} 63$

Loststufe 11

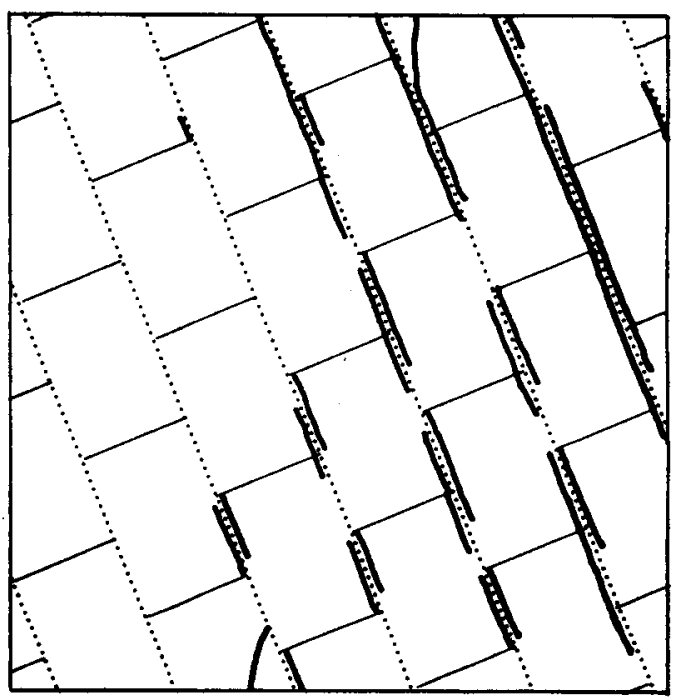

Versuch $\mathrm{K} 53$

Loststufe 9

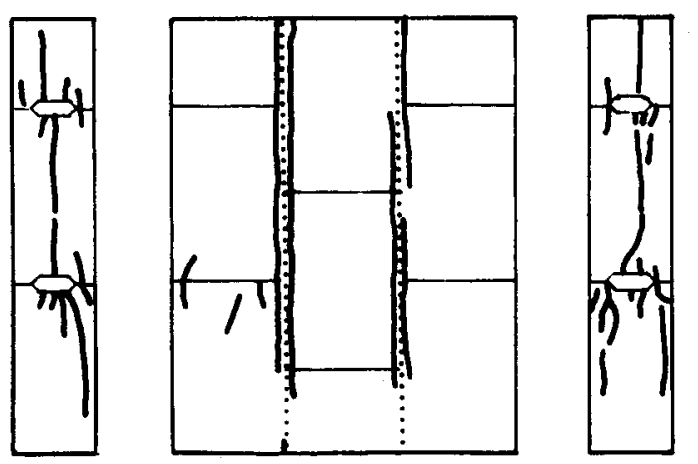

Bild 15: Rissbilder der Versuchsserie 2 
Loststufe 6:

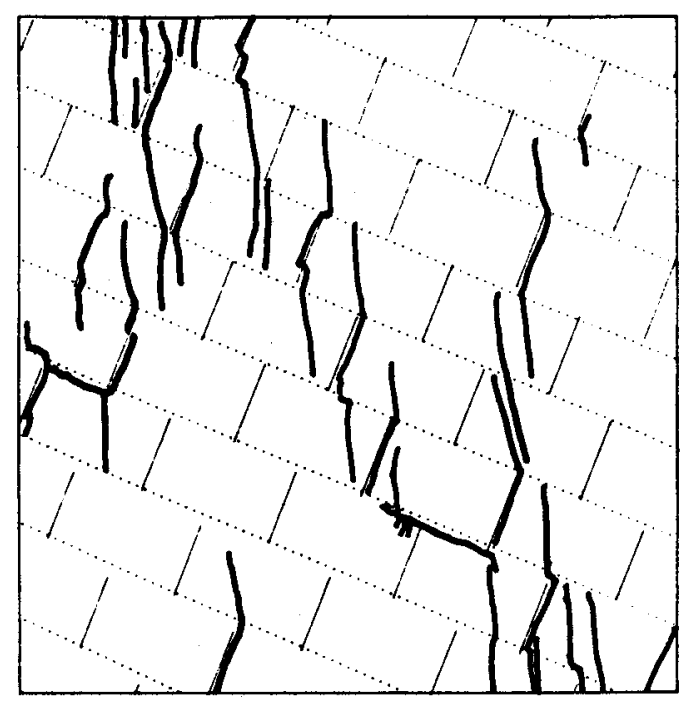

Laststufe 7 :

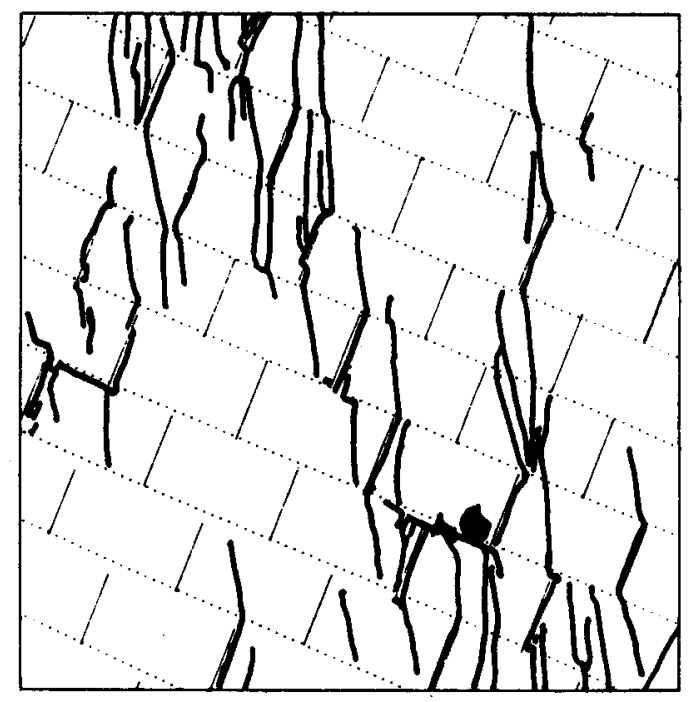

Loststufe 8 :

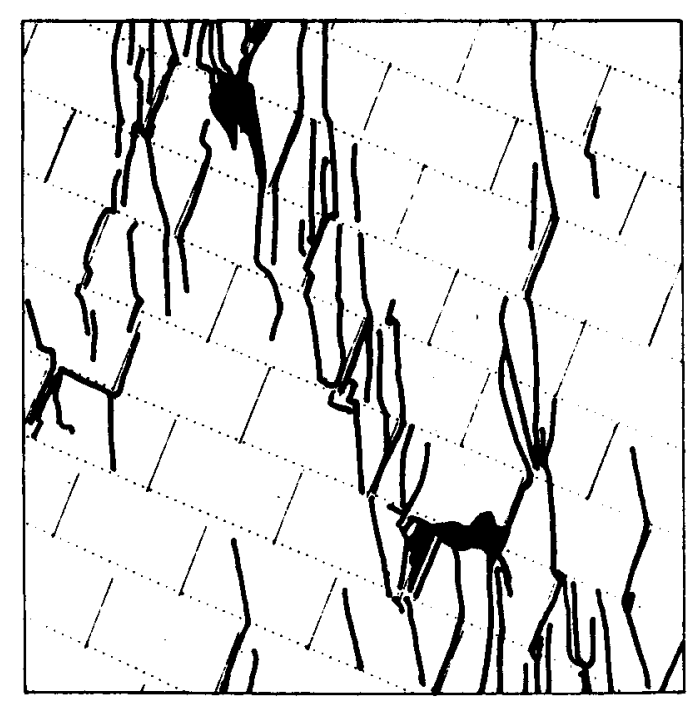

Bild 16: Beispiel für die Rissentwicklung, Versuch K44 
Versuch $\mathrm{K} 22$

Loststufe 10

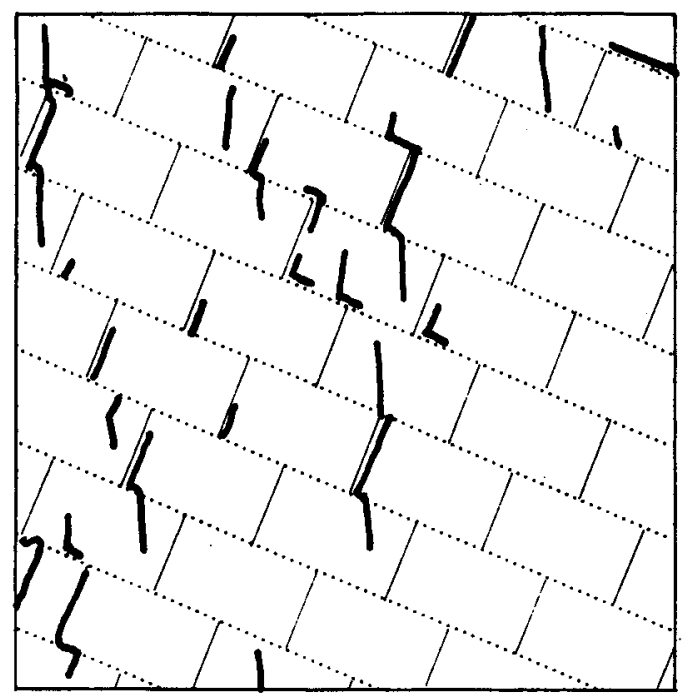

Versuch $\mathrm{K} 30$

Laststufe 11

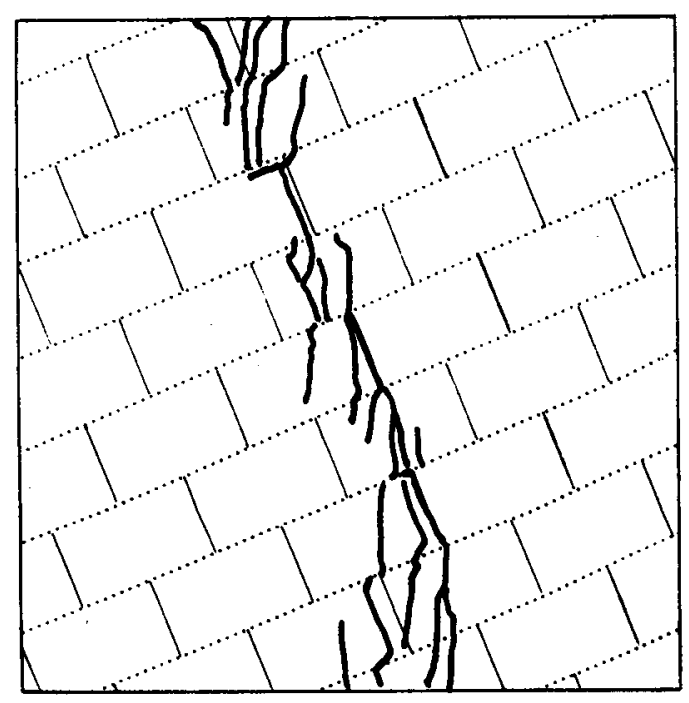

Versuch $\mathrm{K} 26$

Loststufe 8

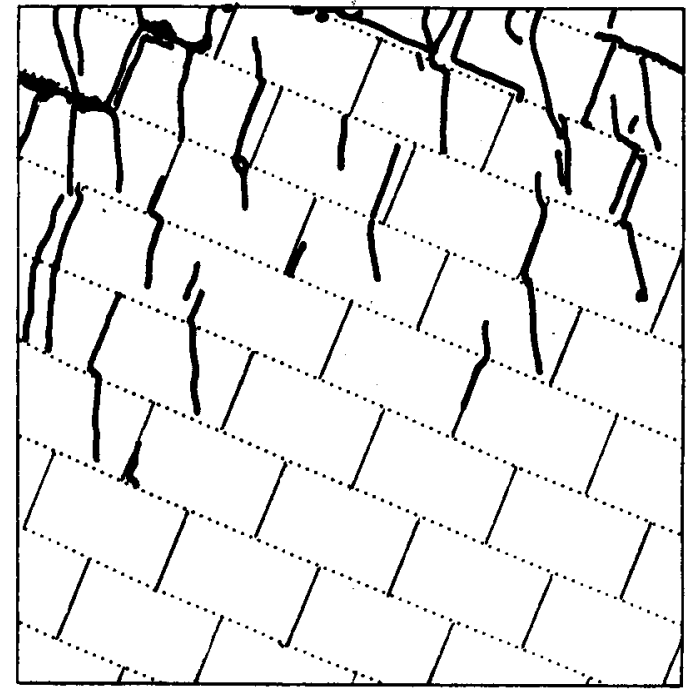

Versuch K38

Laststufe 8

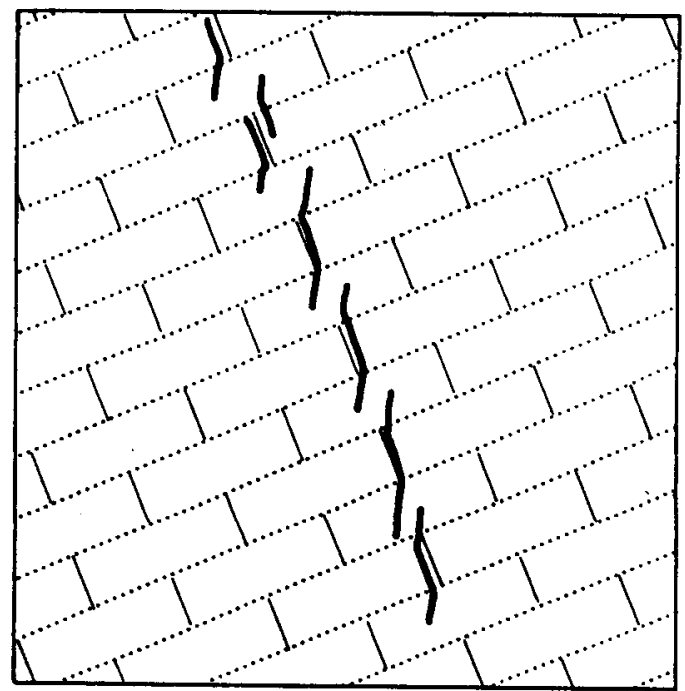

Bild 17: Rissbilder verschiedener Versuche mit $22.5^{\circ}$ Fugenneigung 
Versuch $\mathrm{K} 21$

Laststufe 9

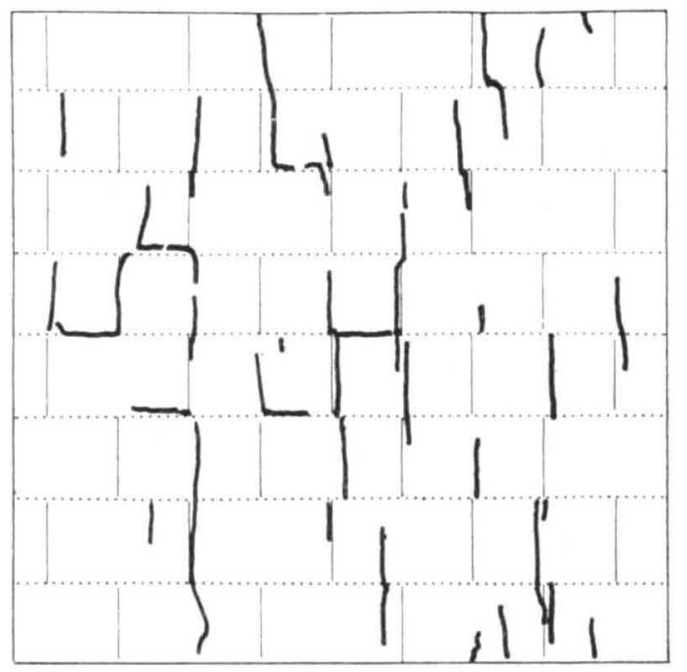

Versuch K28

Laststufe 7

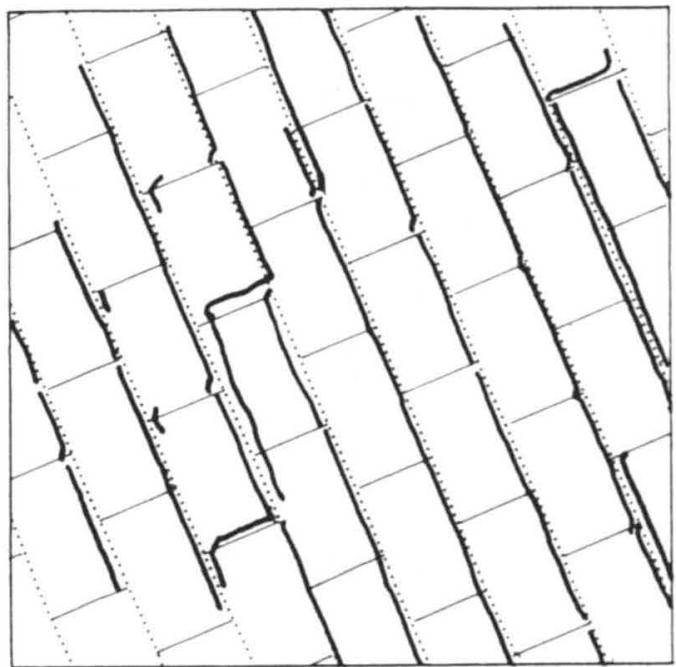

Bild 18: Rissbilder, Versuche $\mathrm{K} 21$ und $\mathrm{K} 28$

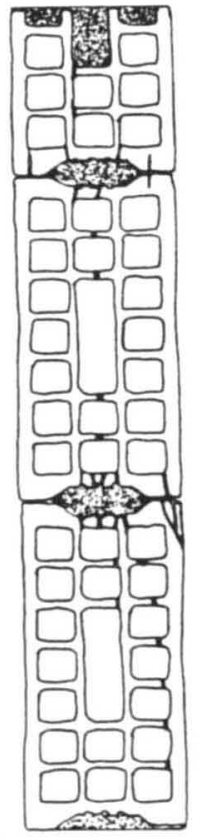

Versuch

Laststufe

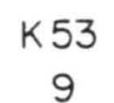

9

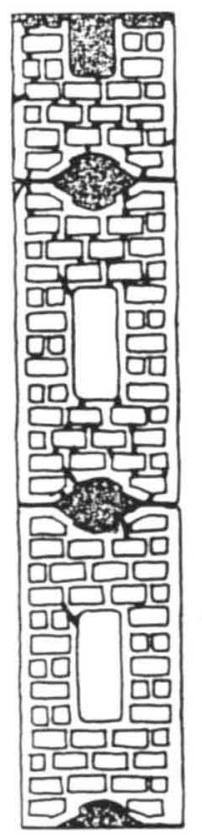

K54

6

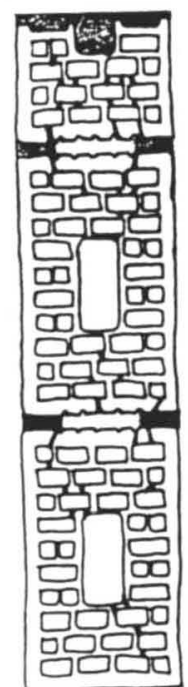

K57

6

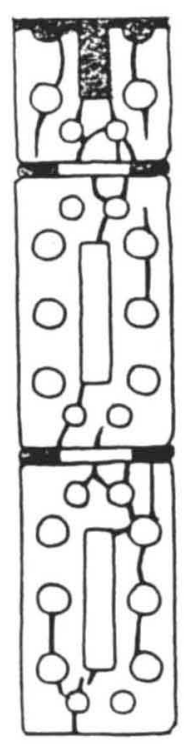

$$
\mathrm{K} 60
$$

14

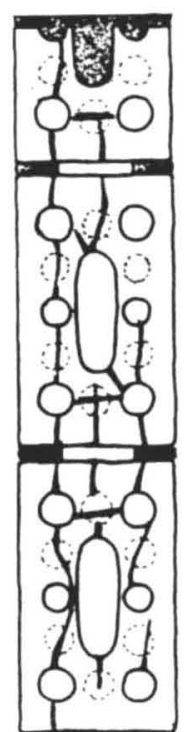

K 61

10

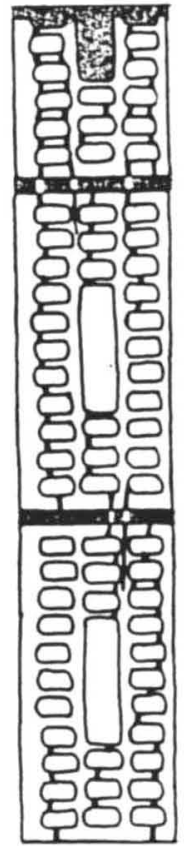

K 59

9

Bild 19: Gerissene Stirnseiten einiger 90-Körper 


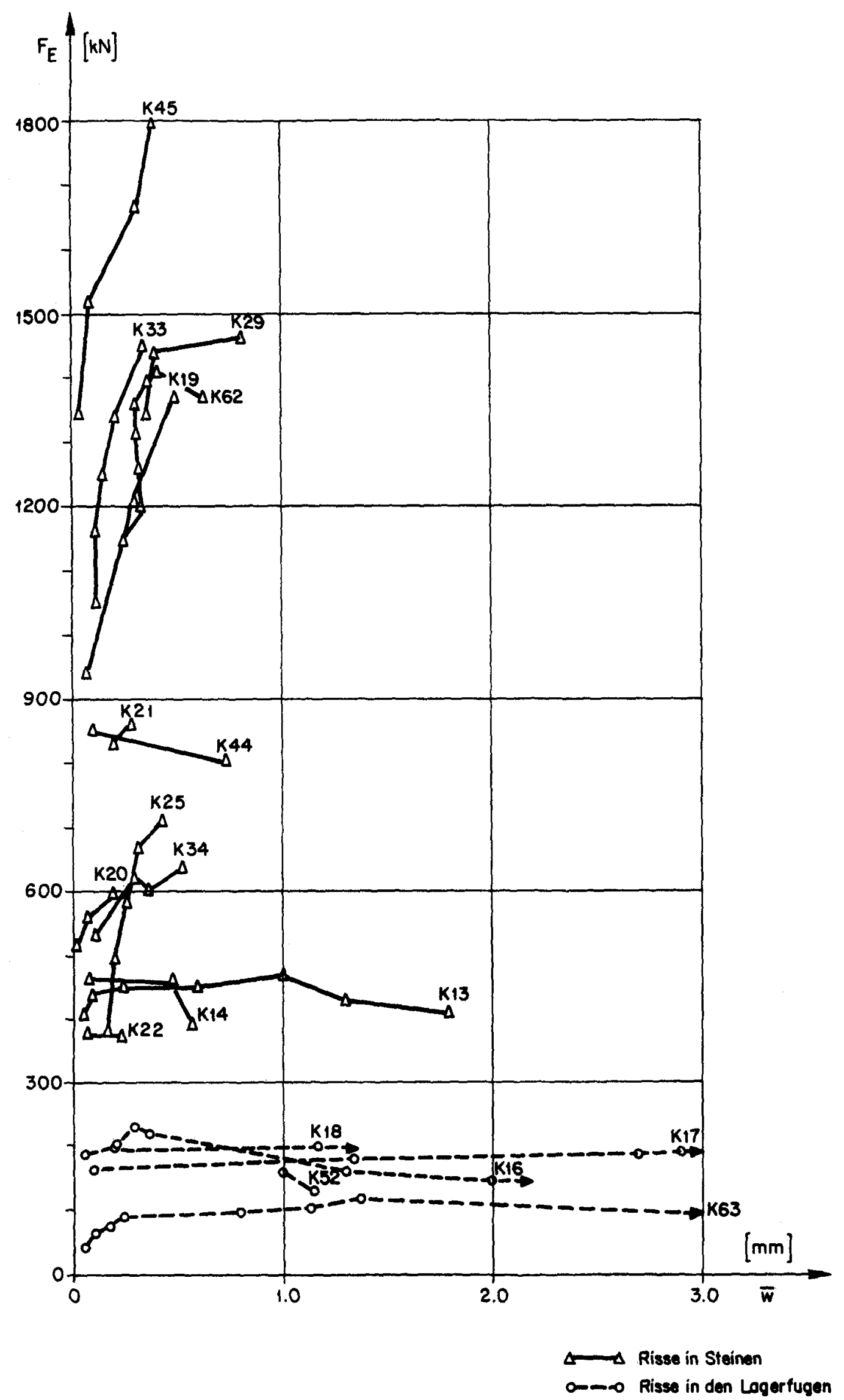

Bild 20: Mittlere Rissweiten 


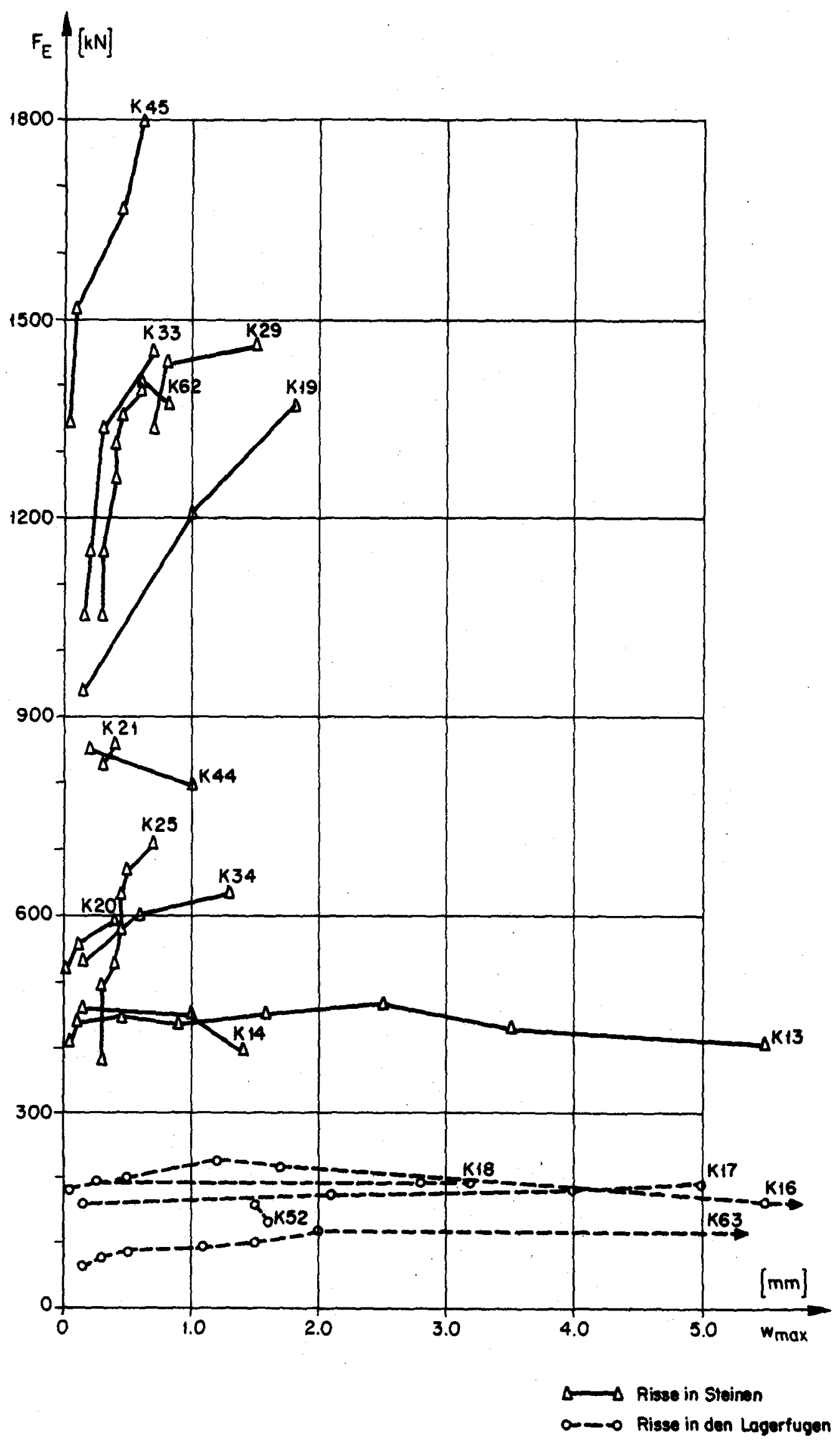

Bild 21: Maximale Rissweiten 

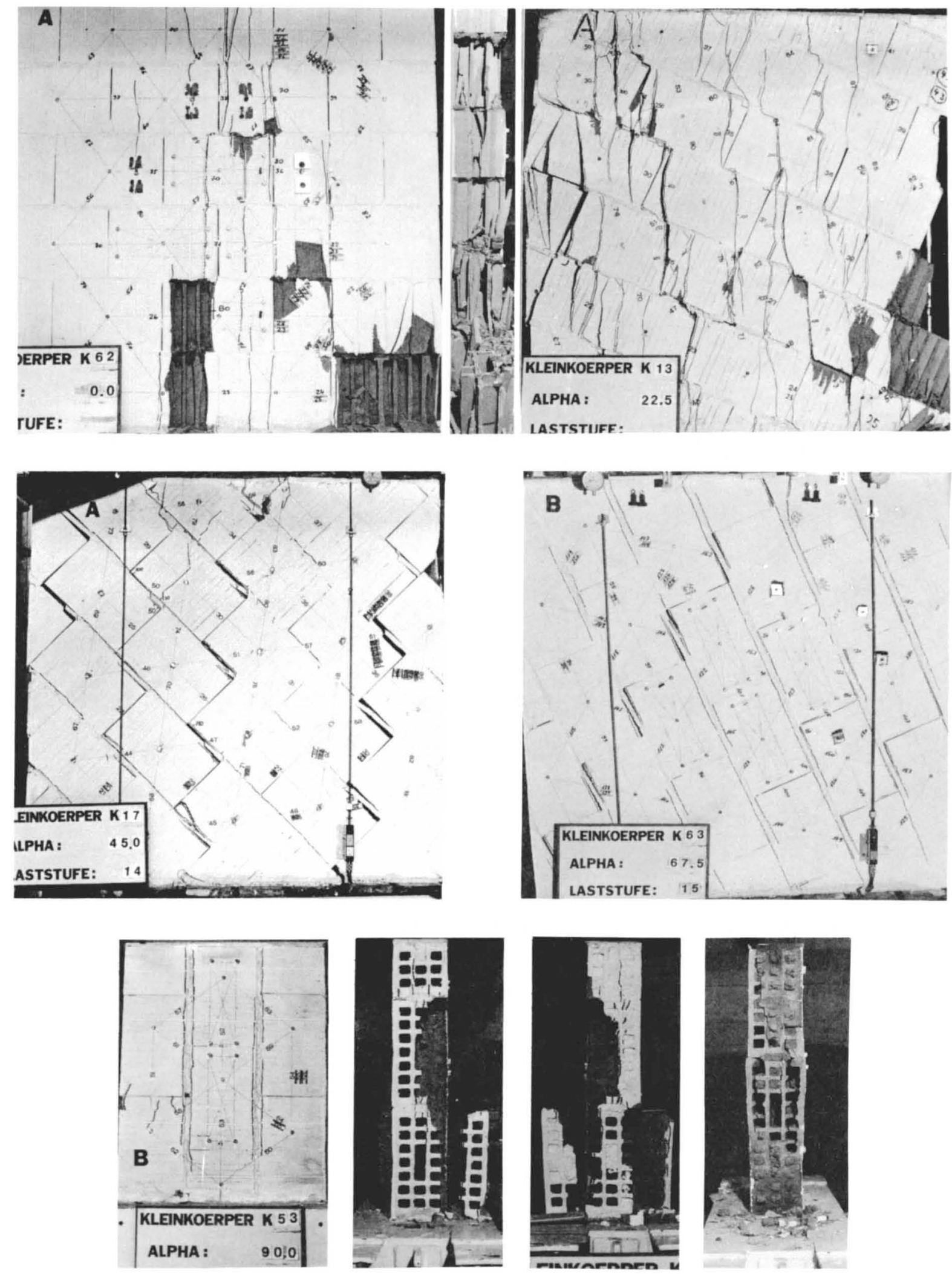

Bild 22: Bruchbilder der Versuchsserie 2 


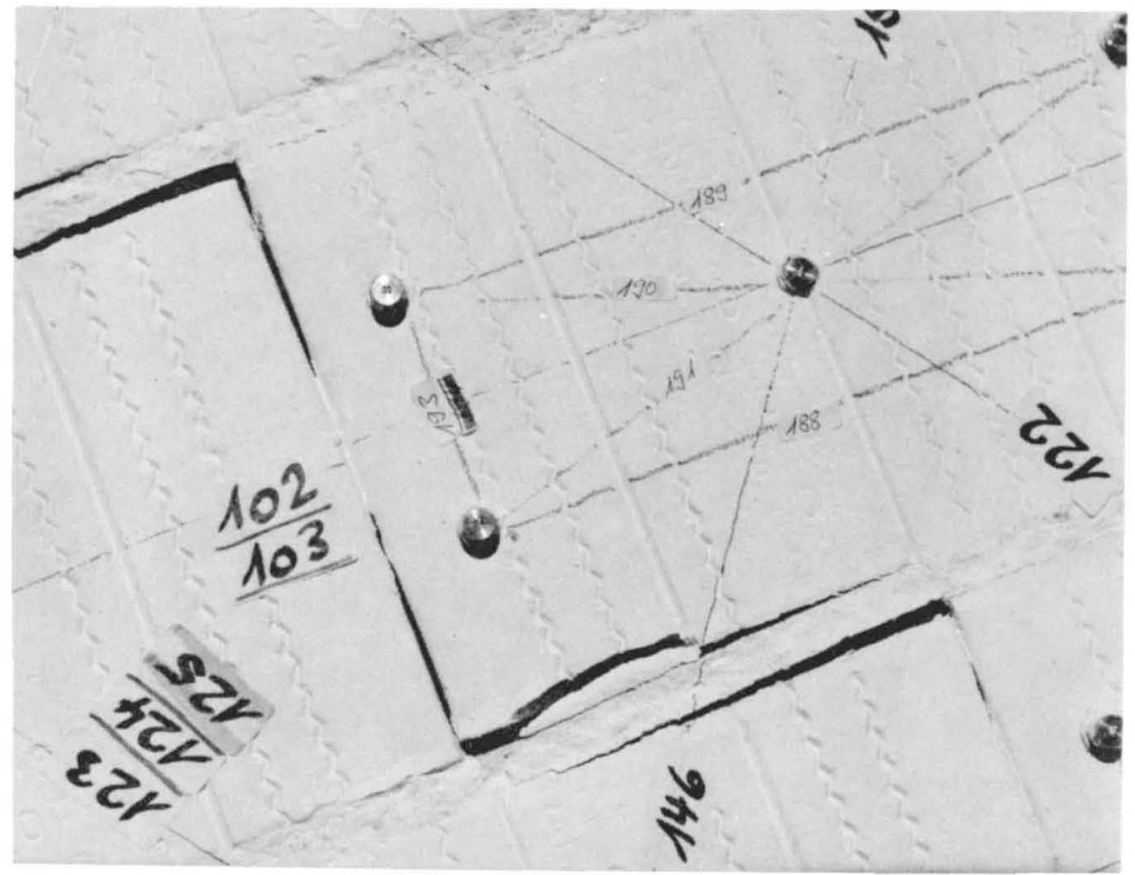

Bild 23: Gleiten der Stossfuge
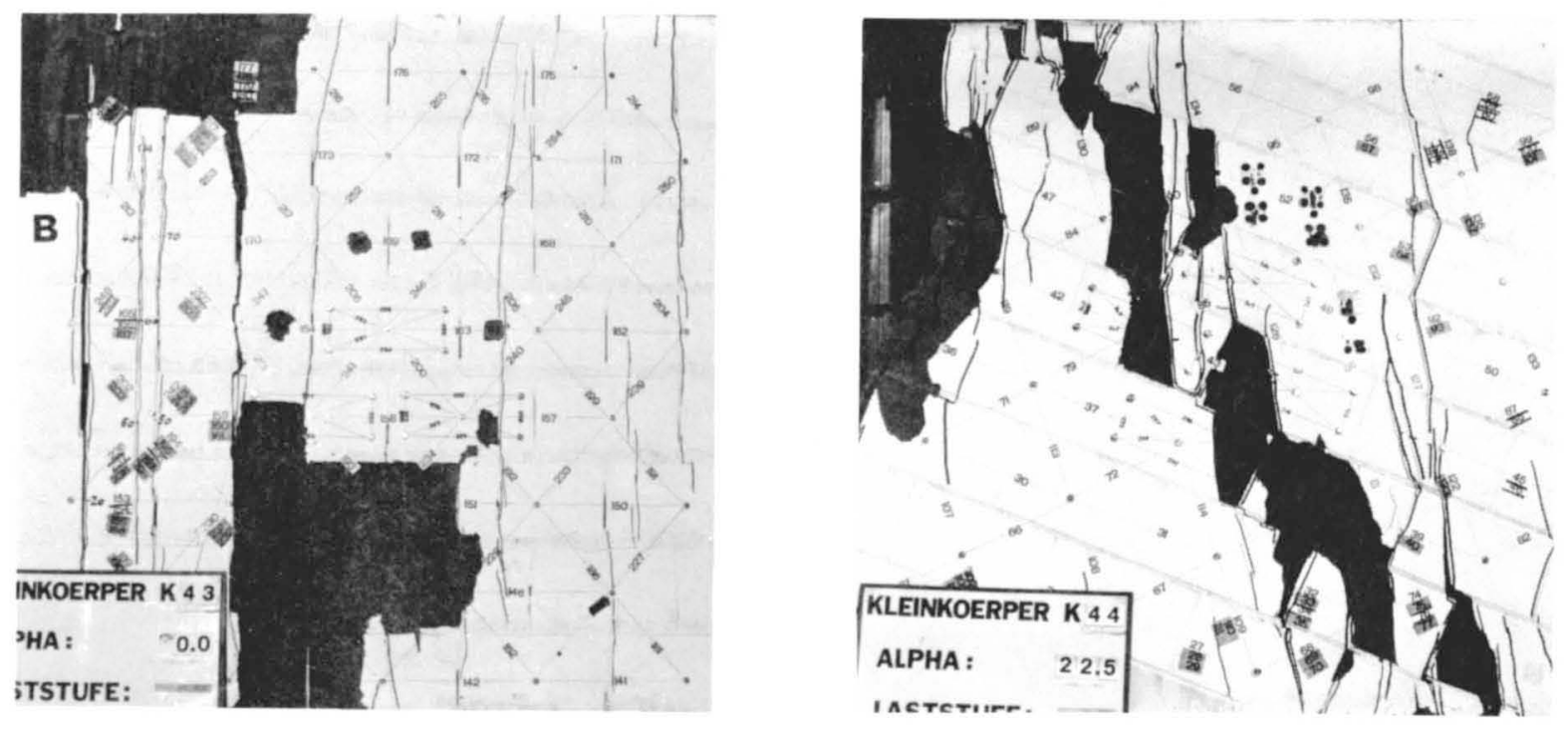

Bild 24: Bruchbilder der Versuchsserie 10 

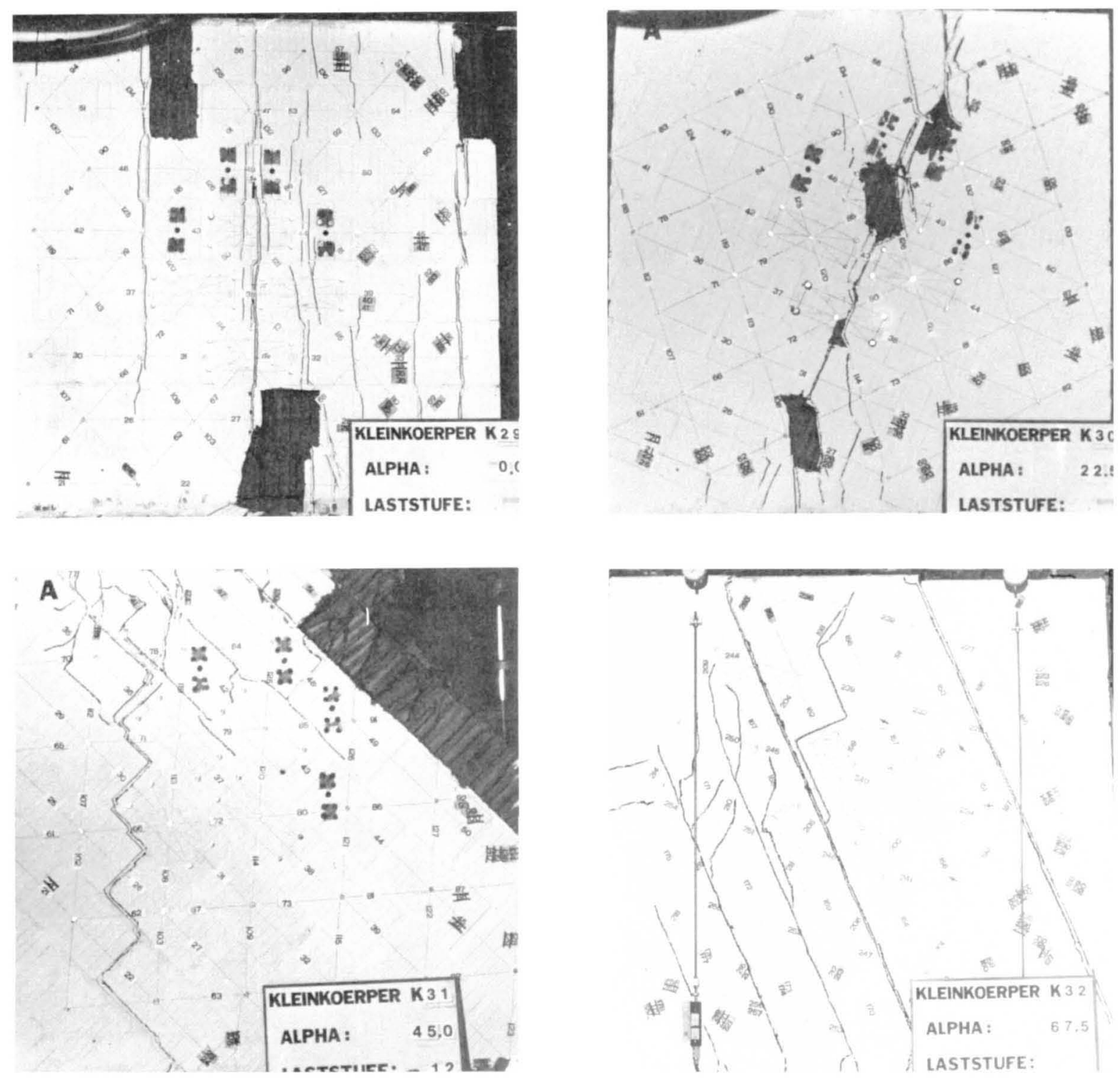

Bild 25: Bruchbilder der Versuchsserie 6 

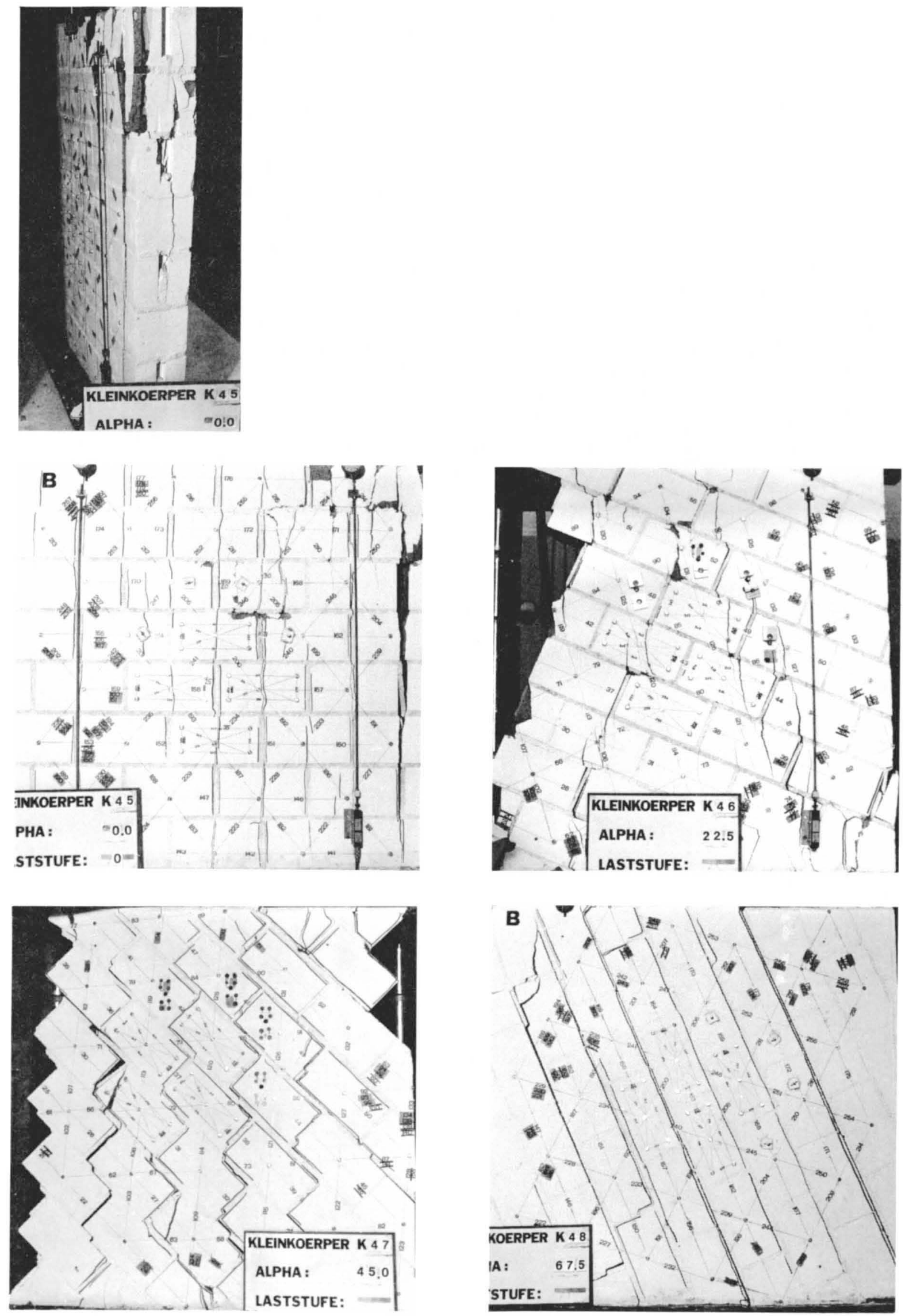

Bild 26: Bruchbilder der Versuchsserie 11 


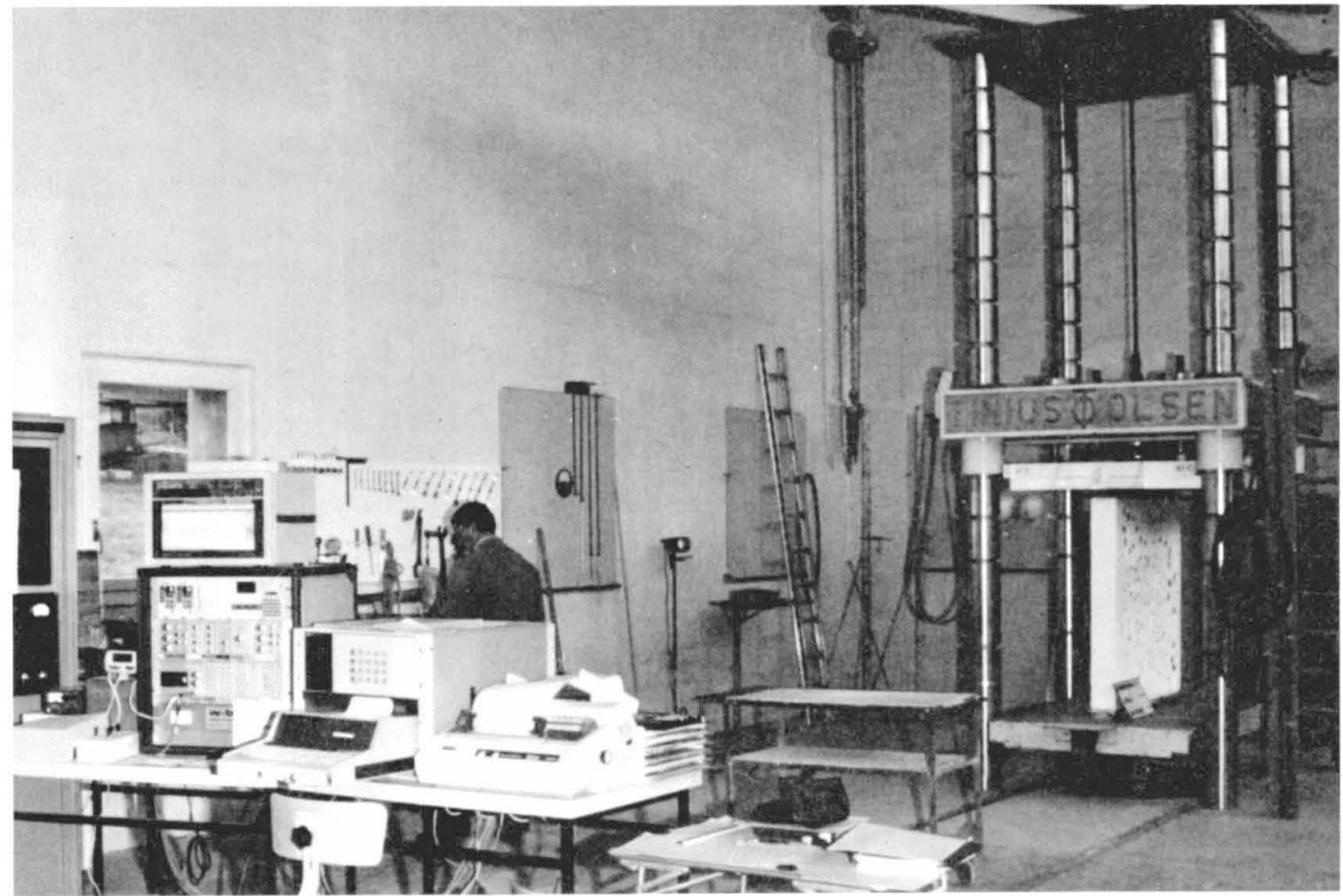

Bild 27: Versuchsanlage: Presse, Steuerung und Datenerfassung

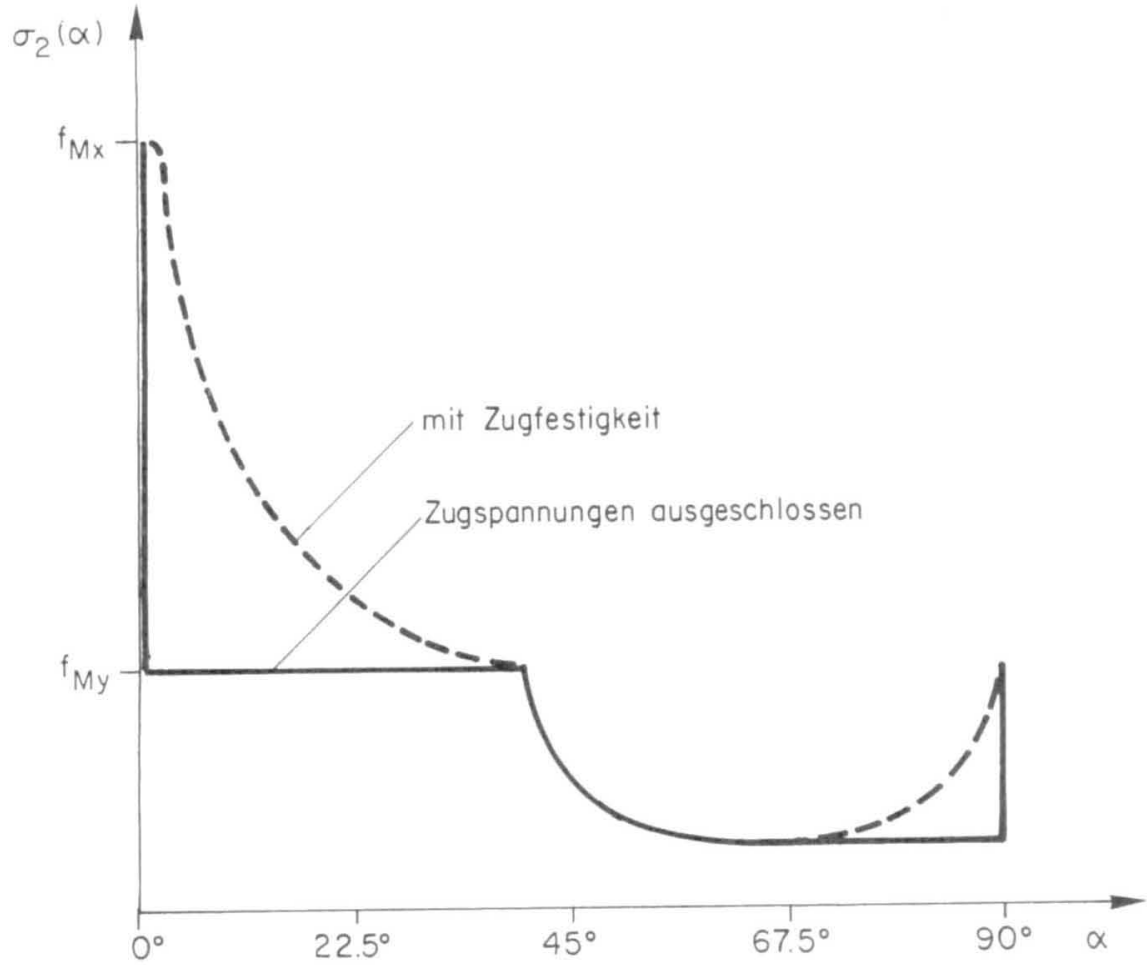

Bild 28: Einfluss der Zugfestigkeit auf die einachsige Festigkeit 


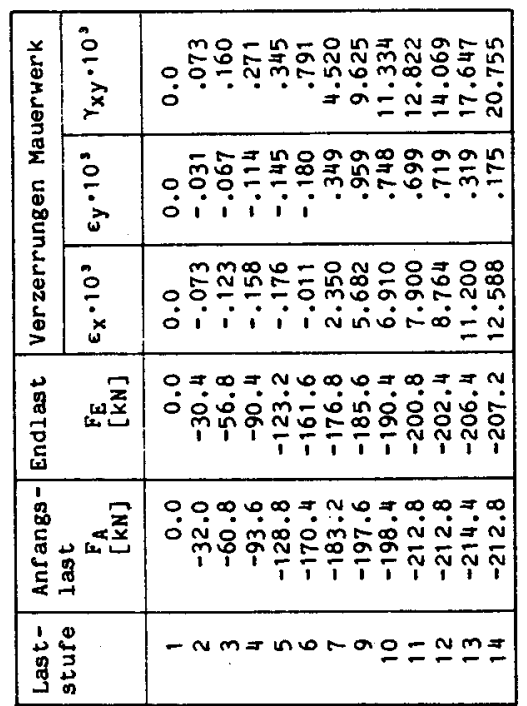

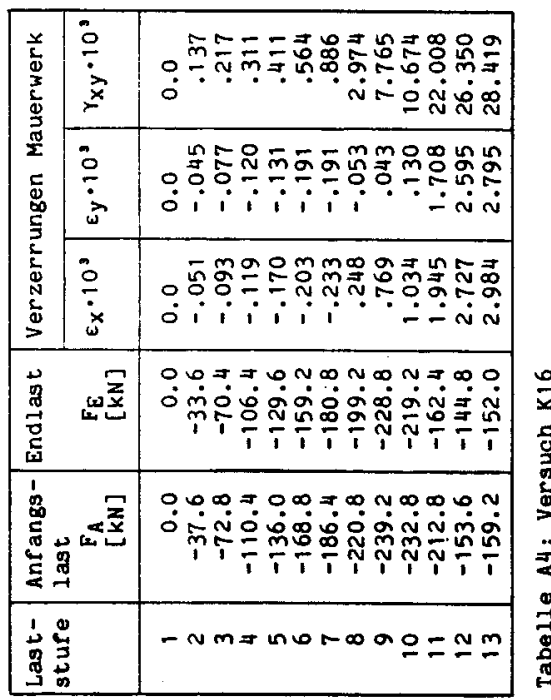

\begin{tabular}{|c|c|c|}
\hline d & $\frac{i}{\bar{x}}$ & 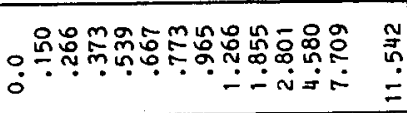 \\
\hline $\begin{array}{l}\Sigma \\
\Sigma \\
\Phi\end{array}$ & $\frac{j}{\dot{\omega}}$ & 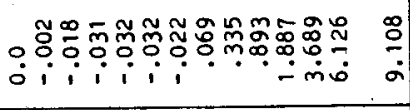 \\
\hline 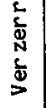 & $\frac{0}{0}$ & 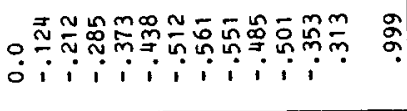 \\
\hline & 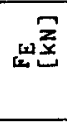 & 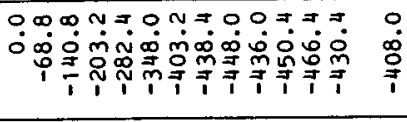 \\
\hline 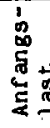 & $\underset{\Sigma}{\bar{z}}$ & 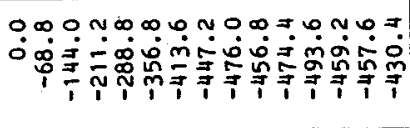 \\
\hline 晜 & & \\
\hline
\end{tabular}
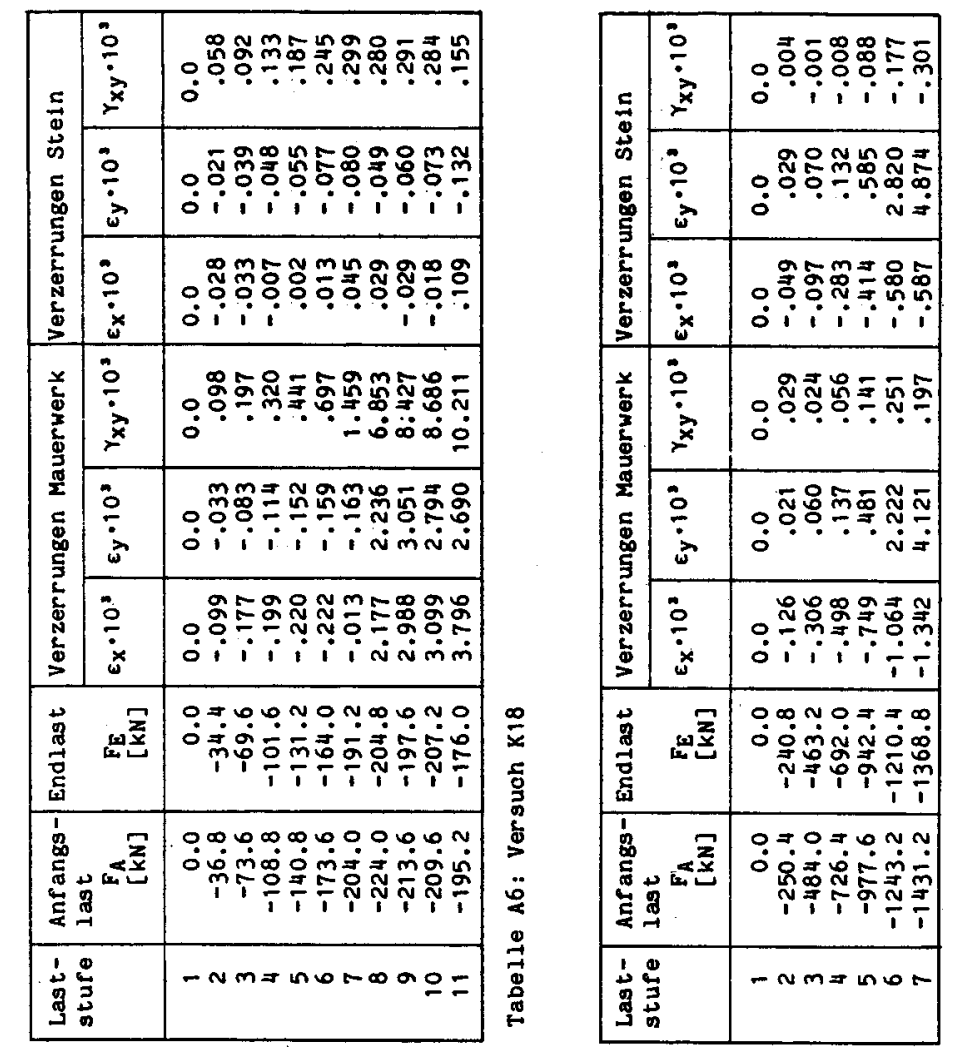

\begin{tabular}{|c|c|c|}
\hline \multirow{2}{*}{ 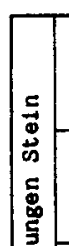 } & 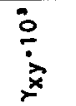 & 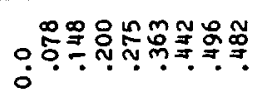 \\
\hline & $\frac{0}{3}$ & 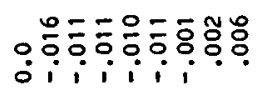 \\
\hline 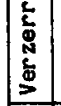 & $\frac{0}{\dot{x}}$ & 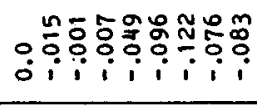 \\
\hline \multirow{2}{*}{ 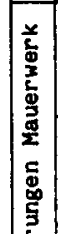 } & $\frac{0}{0}$ & 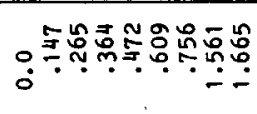 \\
\hline & $\frac{0}{0}$ & 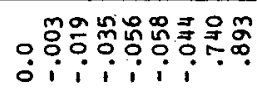 \\
\hline $\mid$ & $\frac{0}{\dot{x}}$ & 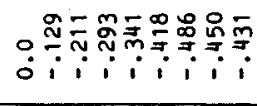 \\
\hline 崫 & $\cos _{\cos }^{\bar{Z}}$ & 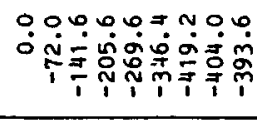 \\
\hline 要 & $\underbrace{<\infty}$ & 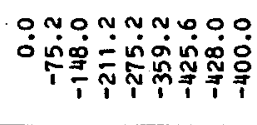 \\
\hline 豆总 & & \\
\hline
\end{tabular}




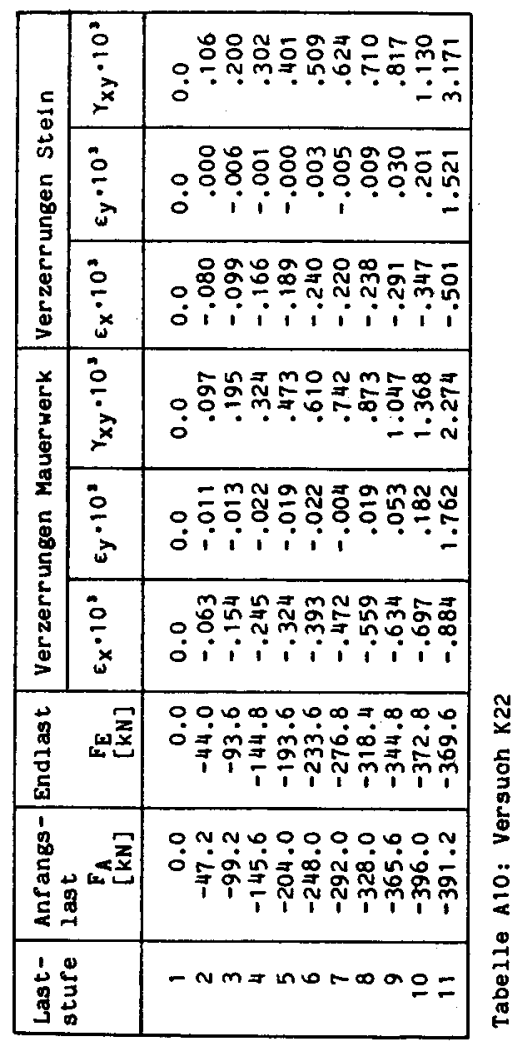

\begin{tabular}{|c|c|}
\hline 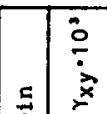 & 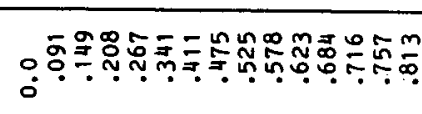 \\
\hline 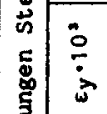 & 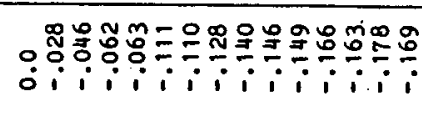 \\
\hline 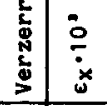 & 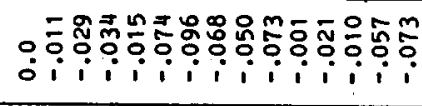 \\
\hline 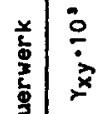 & 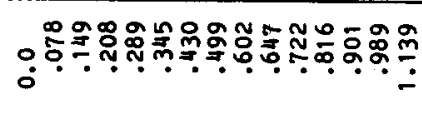 \\
\hline 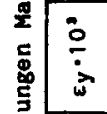 & 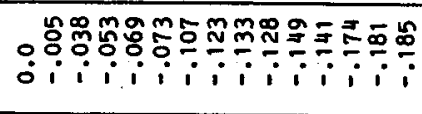 \\
\hline 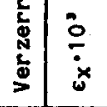 & 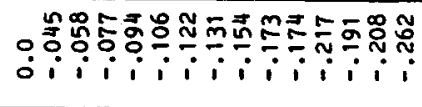 \\
\hline 量 & 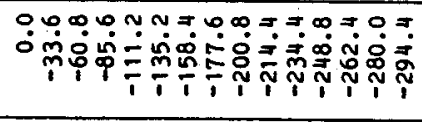 \\
\hline 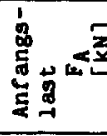 & 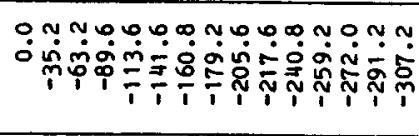 \\
\hline 苟 & - Nmsnon \\
\hline
\end{tabular}

\begin{tabular}{|c|c|}
\hline$=\frac{0}{\vdots}$ & 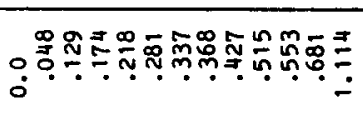 \\
\hline 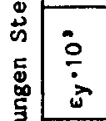 & 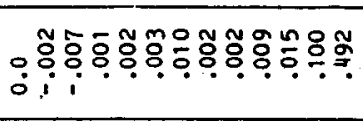 \\
\hline 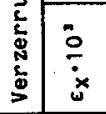 & 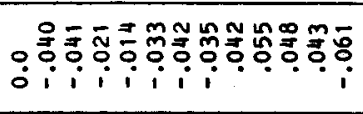 \\
\hline 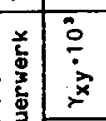 & 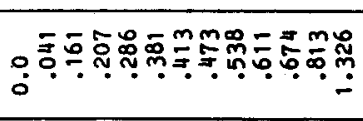 \\
\hline 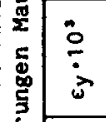 & 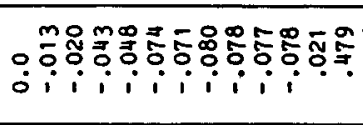 \\
\hline 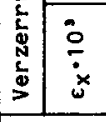 & 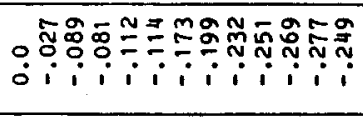 \\
\hline 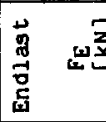 & 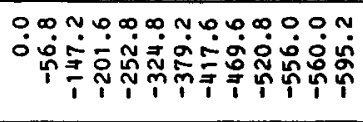 \\
\hline 总事蛋 & 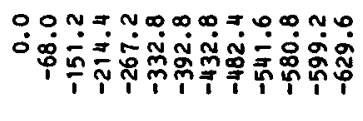 \\
\hline 岁量苛 & $-N N$ \\
\hline
\end{tabular}

\begin{tabular}{|c|c|c|}
\hline 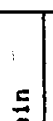 & $\frac{\dot{0}}{\dot{x}}$ & ن \\
\hline 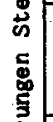 & $\frac{0}{\vdots}$ & ن \\
\hline | & $\frac{\vdots}{\dot{x}}$ & 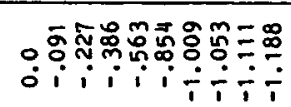 \\
\hline 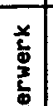 & 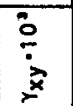 & 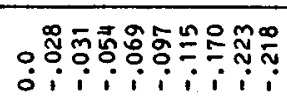 \\
\hline 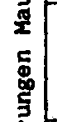 & $\frac{0}{2}$ & 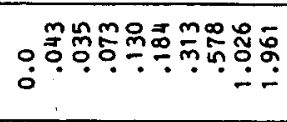 \\
\hline 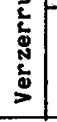 & : & 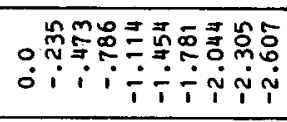 \\
\hline & $\underline{n}$ & 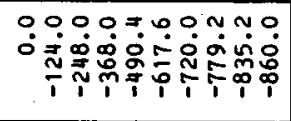 \\
\hline & $=\frac{\pi}{z}$ & 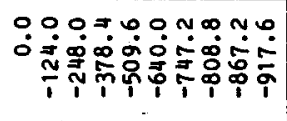 \\
\hline 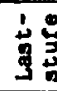 & & $-\sim m$ \\
\hline
\end{tabular}




\begin{tabular}{|c|c|c|}
\hline$\Xi$ & 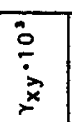 & 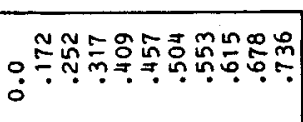 \\
\hline 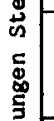 & $\begin{array}{l}0 \\
\vdots \\
\vdots \\
\end{array}$ & 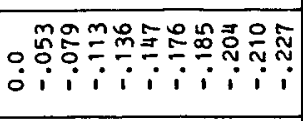 \\
\hline 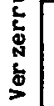 & $\frac{\vdots}{\dot{x}}$ & 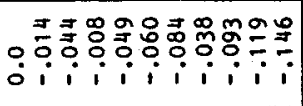 \\
\hline 总 & $\mid$ & 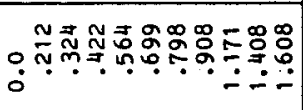 \\
\hline 怔 & $\begin{array}{l}0 \\
\\
3\end{array}$ & 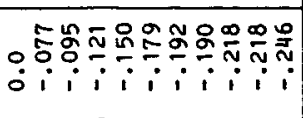 \\
\hline 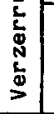 & $\frac{j}{\dot{x}}$ & 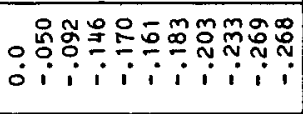 \\
\hline & $\overline{\operatorname{wis}}$ & 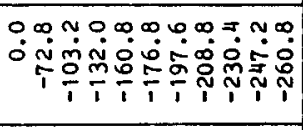 \\
\hline 安 & $=<\frac{7}{3}$ & 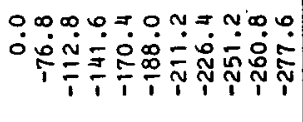 \\
\hline 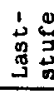 & & or-oos $=$ \\
\hline
\end{tabular}

\begin{tabular}{|c|c|c|}
\hline & $\frac{\overline{0}}{0}$ & 芯 \\
\hline $\begin{array}{l}n \\
\vdots \\
\mathbf{s}\end{array}$ & $\frac{0}{3}$ & 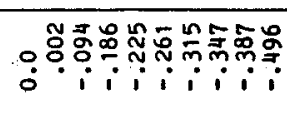 \\
\hline 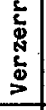 & $\frac{\dot{0}}{\dot{x}}$ & 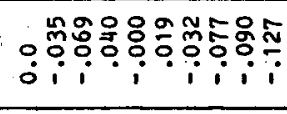 \\
\hline 莺 & $\frac{0}{0}$ & $\mathfrak{O}_{0}^{0}$ \\
\hline $\begin{array}{l}\frac{\mathrm{w}}{2} \\
\overline{\mathrm{w}} \\
\mathrm{w} \\
\mathrm{s}\end{array}$ & $\frac{\vdots}{\vdots}$ & 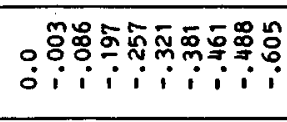 \\
\hline 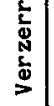 & $\frac{\overline{0}}{\dot{x}}$ & ن \\
\hline & & هُ \\
\hline & $=0$ & 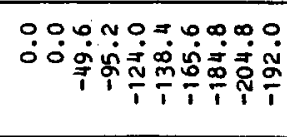 \\
\hline 焉: & & $-\sim m$ \\
\hline
\end{tabular}

\begin{tabular}{|c|c|}
\hline$\frac{0}{\dot{x}}$ & 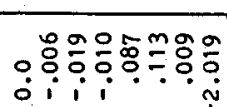 \\
\hline 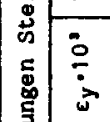 & 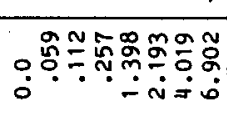 \\
\hline 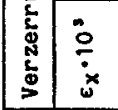 & 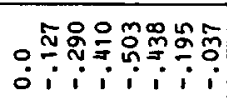 \\
\hline 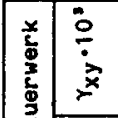 & iَ \\
\hline 童 & 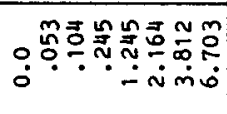 \\
\hline 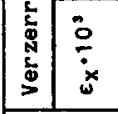 & 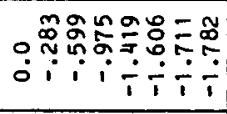 \\
\hline$\vec{g}$ & 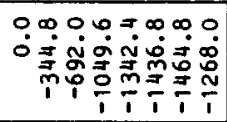 \\
\hline 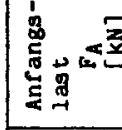 & 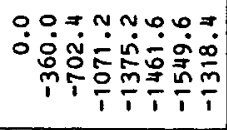 \\
\hline 㻤营 & \\
\hline
\end{tabular}

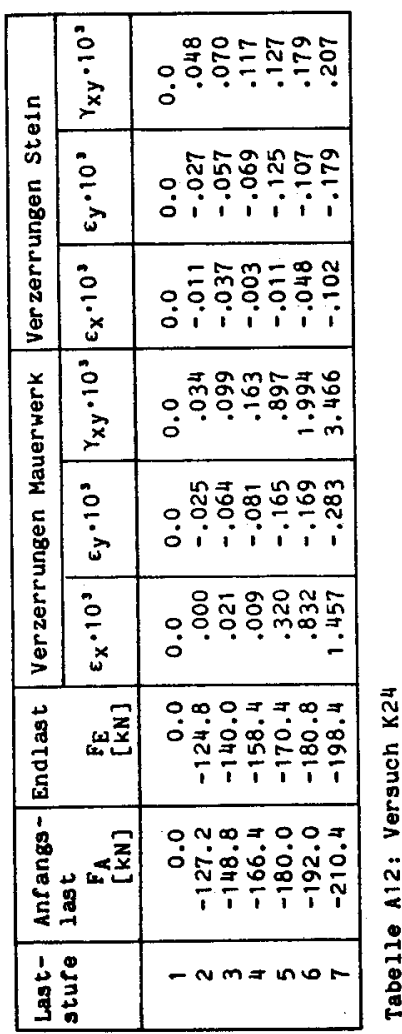

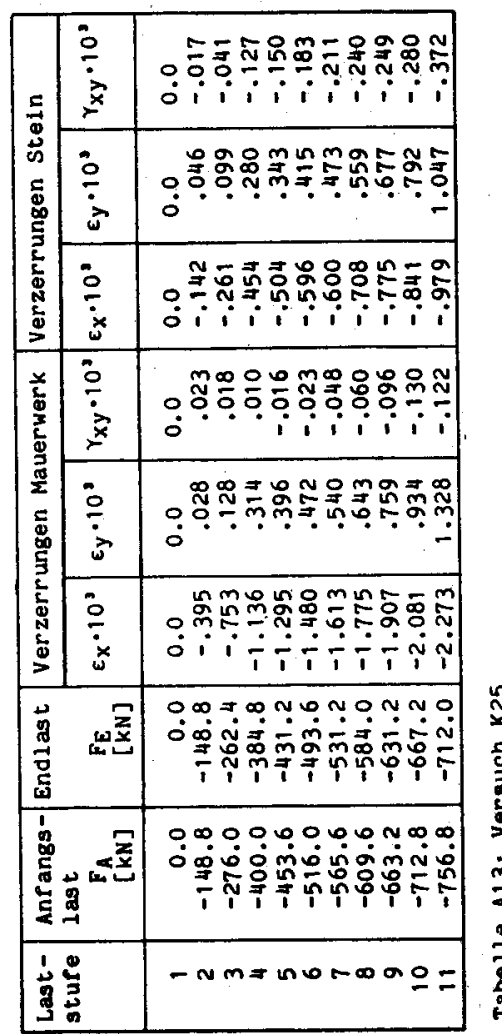

\begin{tabular}{|c|c|c|}
\hline$=$ & 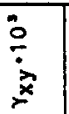 & 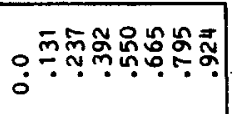 \\
\hline 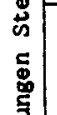 & $\begin{array}{l}\vdots \\
\vdots \\
\vdots \\
\vdots \\
\vdots\end{array}$ & 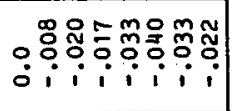 \\
\hline 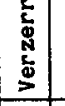 & $\frac{\dot{0}}{\dot{x}}$ & 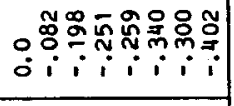 \\
\hline 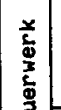 & 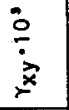 & 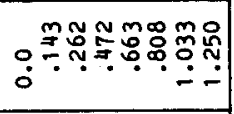 \\
\hline 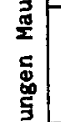 & 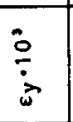 & 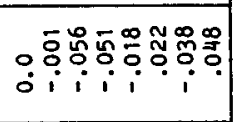 \\
\hline 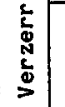 & $\frac{\dot{b}}{\dot{x}}$ & 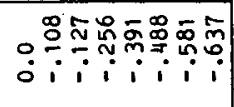 \\
\hline 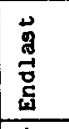 & w言 & 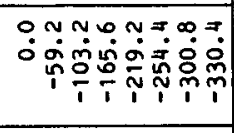 \\
\hline 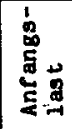 & $0=\frac{\pi}{3}$ & 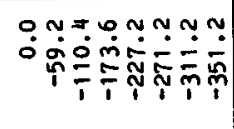 \\
\hline 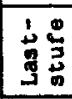 & & $-N m=n \in N \infty$ \\
\hline
\end{tabular}




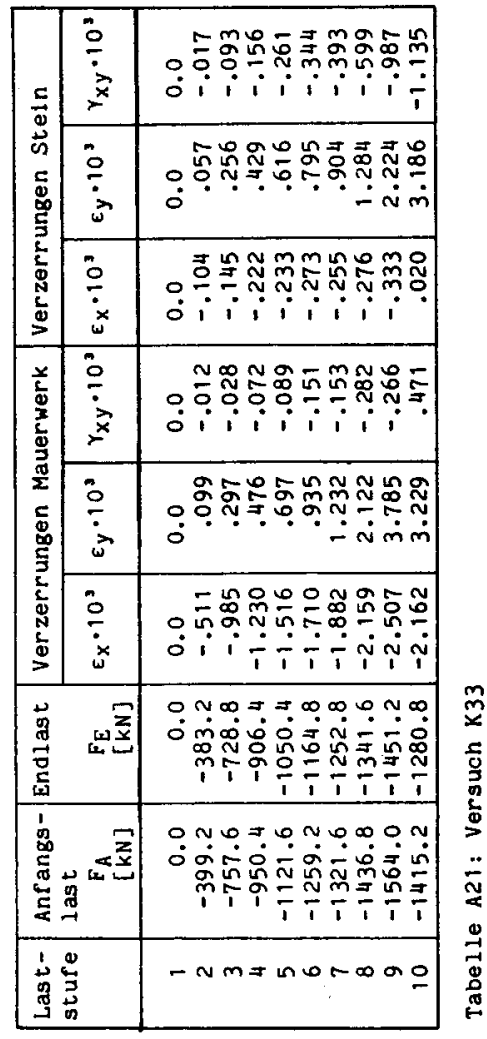

\begin{tabular}{|c|c|}
\hline$=\frac{\dot{0}}{\dot{x}}$ & 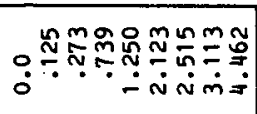 \\
\hline 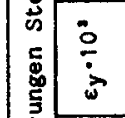 & ن̊ñ \\
\hline 童 & 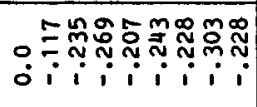 \\
\hline 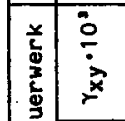 & 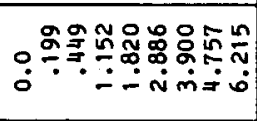 \\
\hline 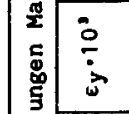 & 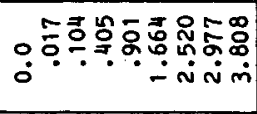 \\
\hline 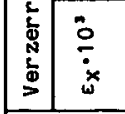 & 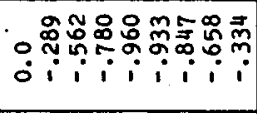 \\
\hline 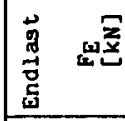 & 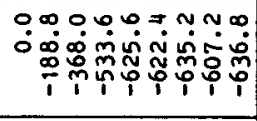 \\
\hline 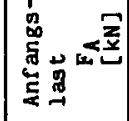 & 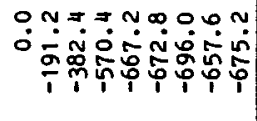 \\
\hline 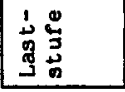 & $-\sim m=n$ \\
\hline
\end{tabular}

\begin{tabular}{|c|c|}
\hline 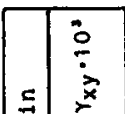 & 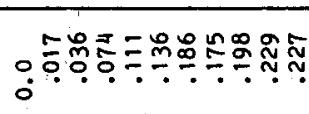 \\
\hline 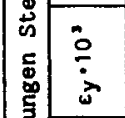 & 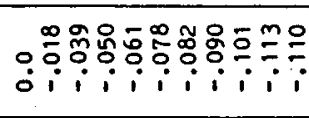 \\
\hline 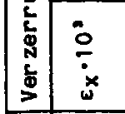 & 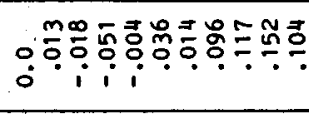 \\
\hline 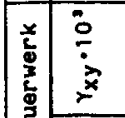 & 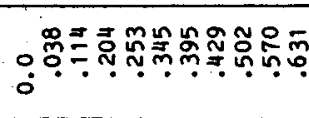 \\
\hline \begin{tabular}{l|l}
$\frac{\pi}{2}$ & 0 \\
$\vdots$ & 0 \\
$\vdots$ & 0 \\
& 0 \\
\end{tabular} & 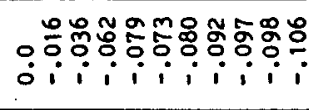 \\
\hline 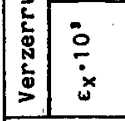 & 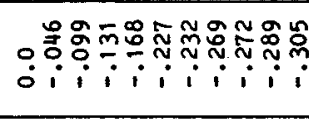 \\
\hline 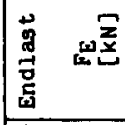 & 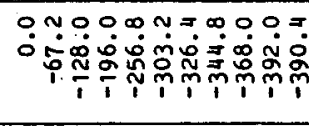 \\
\hline 焉 & 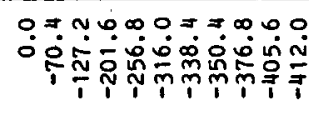 \\
\hline 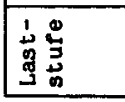 & $-\sim m=$ \\
\hline
\end{tabular}

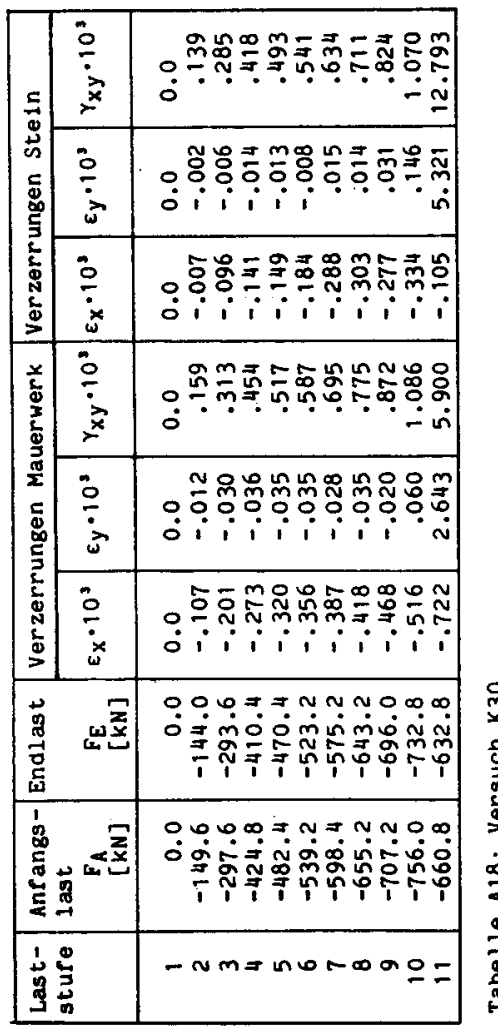

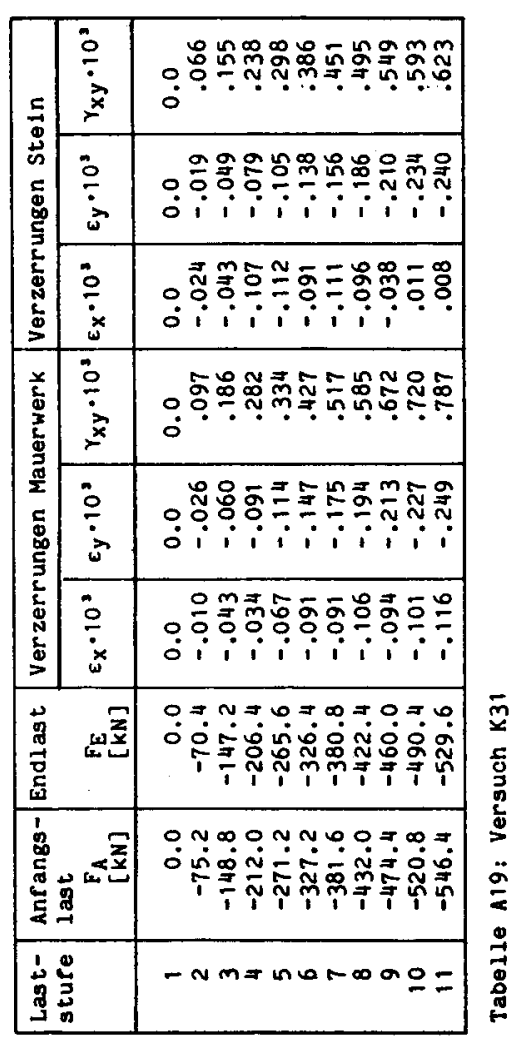

\begin{tabular}{|c|c|c|}
\hline 5 & $\begin{array}{l}\dot{0} \\
\dot{0} \\
\bar{x}\end{array}$ & 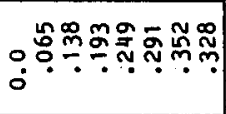 \\
\hline 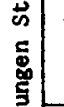 & $\frac{0}{3}$ & 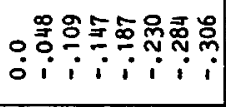 \\
\hline ב. & $\frac{\dot{0}}{\dot{x}}$ & 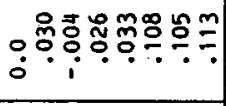 \\
\hline $\mid$ & $\frac{0}{3}$ & 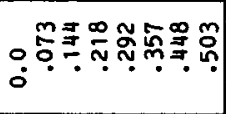 \\
\hline 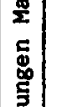 & $\frac{0}{3}$ & 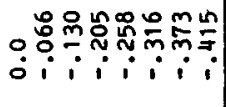 \\
\hline $\mid$ & ì & 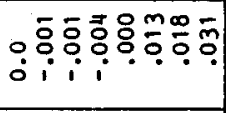 \\
\hline & $\underset{\omega}{\underline{z}}$ & 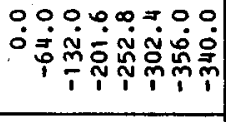 \\
\hline & $=\sqrt[7]{3}$ & 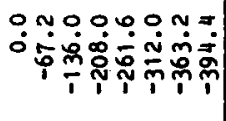 \\
\hline 象 & & \\
\hline
\end{tabular}




\begin{tabular}{|c|c|c|}
\hline \multirow{3}{*}{$\begin{array}{l}5 \\
0 \\
0 \\
0 \\
5 \\
0 \\
0 \\
5 \\
0 \\
0 \\
0 \\
0 \\
0 \\
2\end{array}$} & 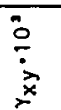 & 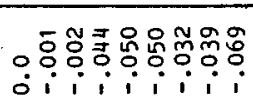 \\
\hline & $\frac{i}{i}$ & 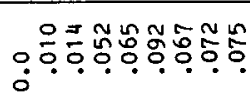 \\
\hline & $\frac{0}{0}$ & 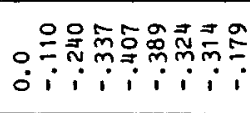 \\
\hline \multirow{3}{*}{ 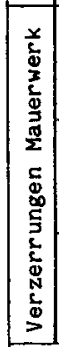 } & 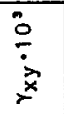 & 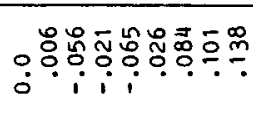 \\
\hline & $\stackrel{0}{0}$ & 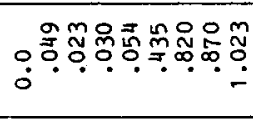 \\
\hline & o & 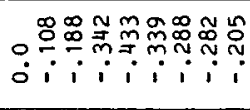 \\
\hline & $\underset{\cos }{\bar{z}}$ & 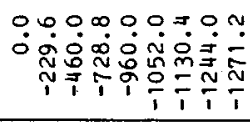 \\
\hline $\begin{array}{l} \\
\\
0 \\
0 \\
0 \\
0 \\
\vdots \\
\vdots \\
\end{array}$ & $\frac{\pi}{2}$ & 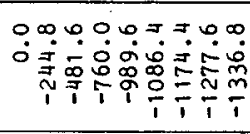 \\
\hline $\begin{array}{l}1 \\
\\
\end{array}$ & & $-\sim m=\ln r-\infty a$ \\
\hline
\end{tabular}

\begin{tabular}{|c|c|c|}
\hline \multirow{3}{*}{ 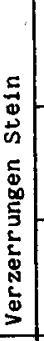 } & $\frac{\dot{0}}{\dot{x}}$ & 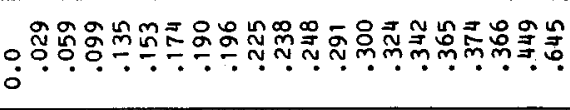 \\
\hline & $\frac{i}{i}$ & 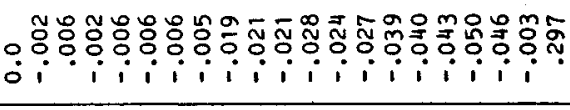 \\
\hline & $\ddot{0}$ & 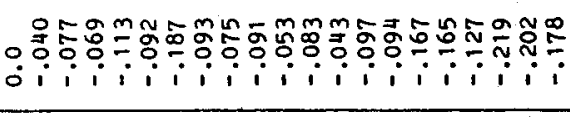 \\
\hline \multirow{3}{*}{ 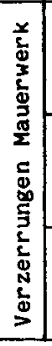 } & $\stackrel{i}{i}$ & 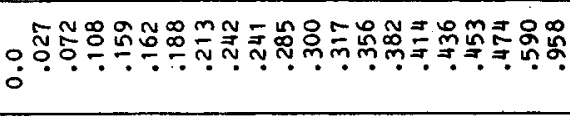 \\
\hline & $\stackrel{0}{\vdots}$ & 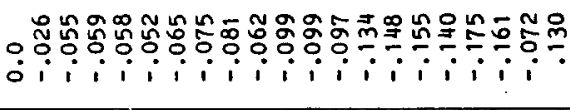 \\
\hline & $\stackrel{0}{0}$ & 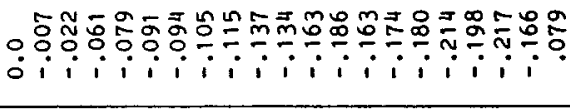 \\
\hline & $\sqrt{3}$ & 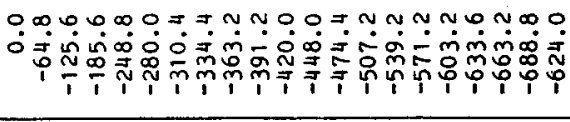 \\
\hline 委 & 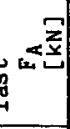 & 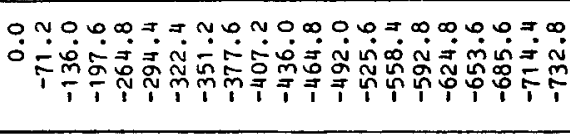 \\
\hline $\begin{array}{l}3 \\
0 \\
0\end{array}$ & & 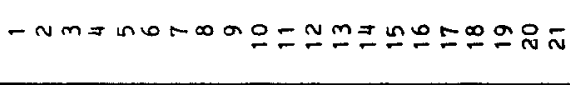 \\
\hline
\end{tabular}

\begin{tabular}{|c|c|c|}
\hline$=$ & \begin{tabular}{l|} 
\\
$\dot{0}$ \\
$\dot{\vec{x}}$ \\
\end{tabular} & 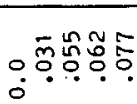 \\
\hline 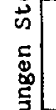 & $\begin{array}{c}0 \\
\vdots \\
\vdots\end{array}$ & o \\
\hline 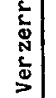 & $\frac{0}{0}$ & 오ำดั \\
\hline 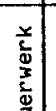 & $\begin{array}{l}0 \\
0 \\
\dot{x} \\
x\end{array}$ & 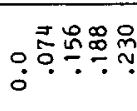 \\
\hline 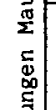 & $\begin{array}{l}0 \\
0 \\
0 \\
0\end{array}$ & 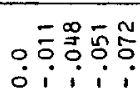 \\
\hline 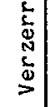 & $\stackrel{0}{0}$ & 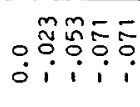 \\
\hline 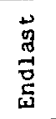 & 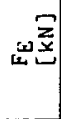 & 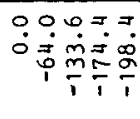 \\
\hline 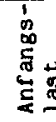 & $\underset{3}{3}$ & ب. \\
\hline 要。 & & $-n m=n$ \\
\hline
\end{tabular}

\begin{tabular}{|c|c|c|}
\hline \pm & $\begin{array}{l}\dot{0} \\
\dot{\vec{x}} \\
\dot{\vec{x}}\end{array}$ & : \\
\hline $\begin{array}{l}5 \\
\vdots \\
5 \\
\$ \\
5 \\
5\end{array}$ & $\stackrel{0}{0}$ & 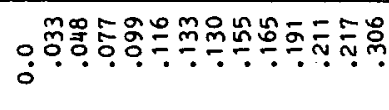 \\
\hline 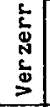 & $\stackrel{\ddot{g}}{\ddot{x}}$ & 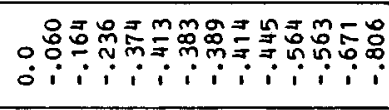 \\
\hline 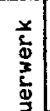 & $\frac{0}{\dot{x}}$ & 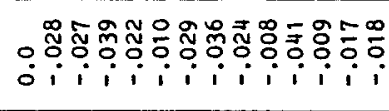 \\
\hline $\begin{array}{c}\frac{\pi}{2} \\
5 \\
5 \\
0 \\
5 \\
5\end{array}$ & $\stackrel{0}{0}$ & 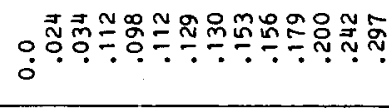 \\
\hline 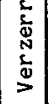 & $\frac{0}{\dot{x}}$ & 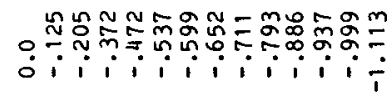 \\
\hline & $\underline{\operatorname{zos}}$ & 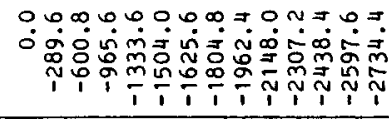 \\
\hline & $=0$ & 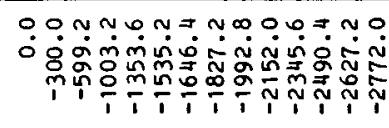 \\
\hline 它 & & $-N m=n \in R \infty a g$ \\
\hline
\end{tabular}

\begin{tabular}{|c|c|c|}
\hline \multirow{3}{*}{ 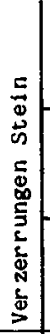 } & 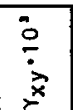 & 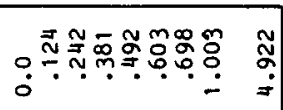 \\
\hline & $\frac{0}{i}$ & $\begin{array}{l}m \infty \\
0 \\
0\end{array}$ \\
\hline & $\frac{\dot{0}}{\dot{0}}$ & 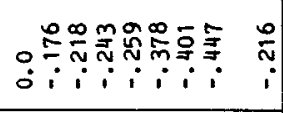 \\
\hline \multirow{3}{*}{ 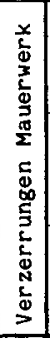 } & $\frac{0}{\dot{x}}$ & 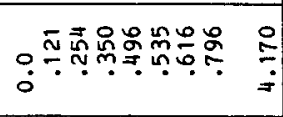 \\
\hline & $\frac{i}{i}$ & 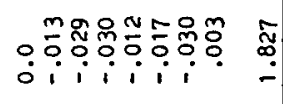 \\
\hline & $\frac{0}{\dot{0}}$ & 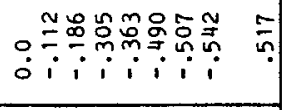 \\
\hline & $\underset{10}{z}$ & 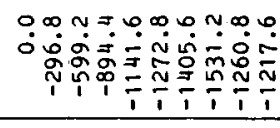 \\
\hline & 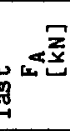 & 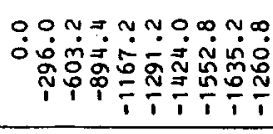 \\
\hline 岢 & & $-N m=\ln 0$ - \\
\hline
\end{tabular}




\begin{tabular}{|c|c|c|}
\hline \multirow[b]{3}{*}{ 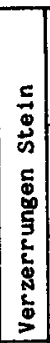 } & $\begin{array}{l}0 \\
\vdots \\
\vdots \\
x\end{array}$ & 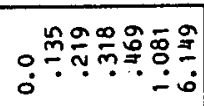 \\
\hline & $\frac{0}{2}$ & 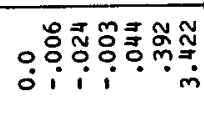 \\
\hline & $\frac{0}{x}$ & 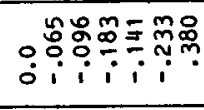 \\
\hline 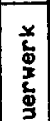 & $\frac{\vdots}{\dot{0}}$ & مَ \\
\hline 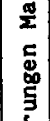 & $\frac{0}{\vdots}$ & 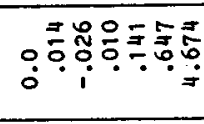 \\
\hline & $\frac{\dot{0}}{\dot{x}}$ & 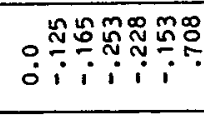 \\
\hline & 뚠 & 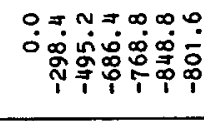 \\
\hline & $\underline{g}$ & 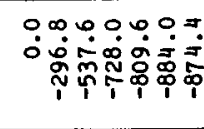 \\
\hline 焉 & & $-\sim m=$ \\
\hline
\end{tabular}

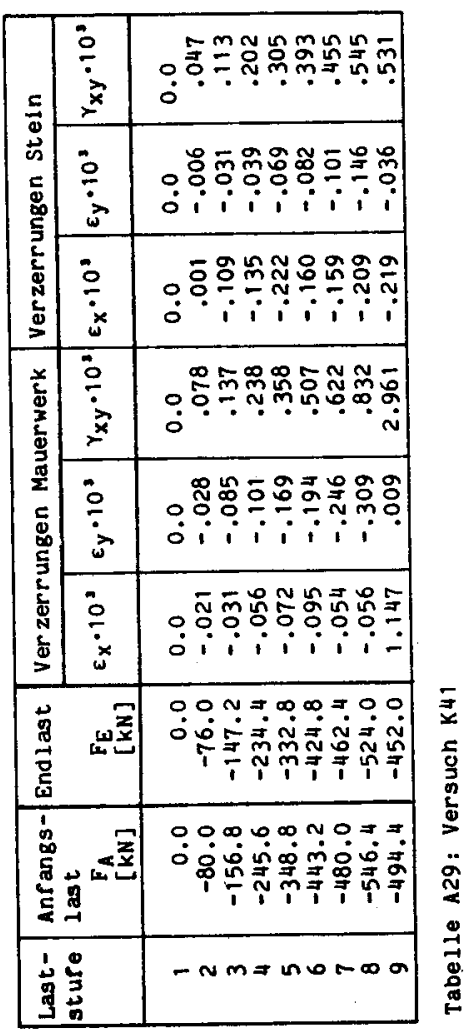

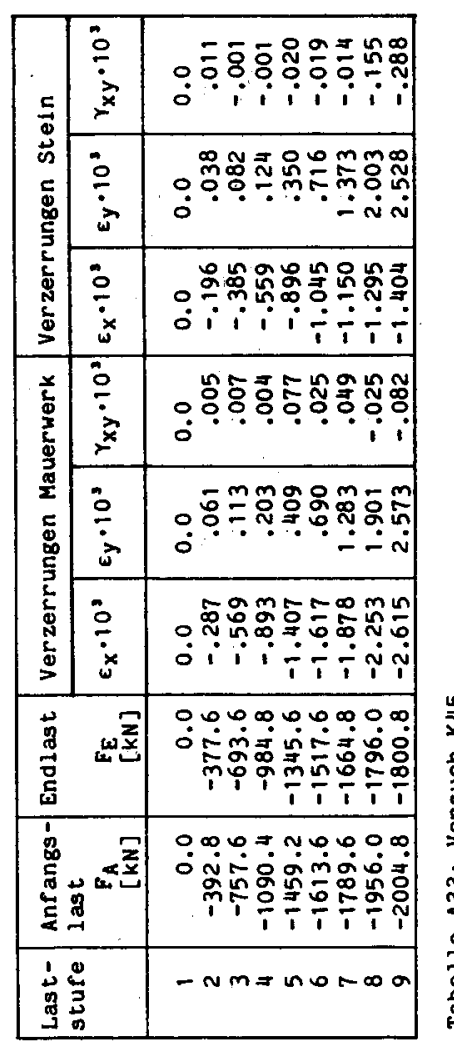

\begin{tabular}{|c|c|c|}
\hline$=$ & 高 & 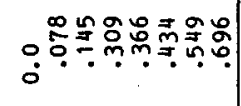 \\
\hline . & $\frac{0}{0}$ & 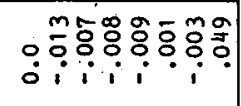 \\
\hline 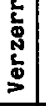 & $\frac{0}{x}$ & 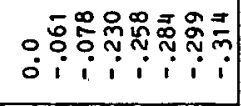 \\
\hline 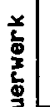 & 立 & 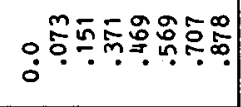 \\
\hline$\left|\begin{array}{l}\frac{w}{2} \\
\frac{5}{0} \\
\frac{5}{3}\end{array}\right|$ & $\frac{0}{i}$ & 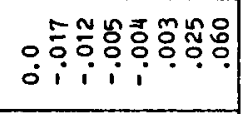 \\
\hline $\mid$ & $\frac{0}{\dot{x}}$ & 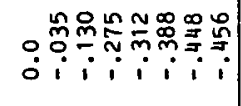 \\
\hline 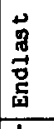 & 百 & 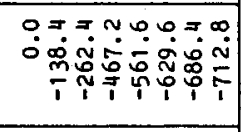 \\
\hline & 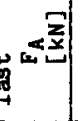 & 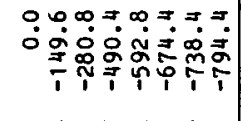 \\
\hline 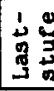 & & $-N m=n \infty-\infty$ \\
\hline
\end{tabular}

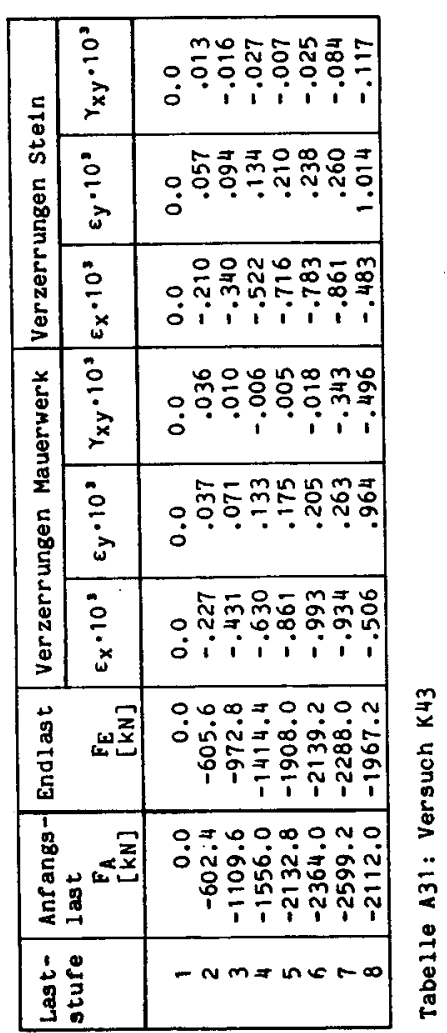




\begin{tabular}{|c|c|}
\hline 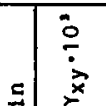 & 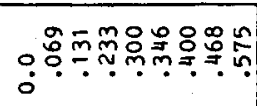 \\
\hline 竧 & 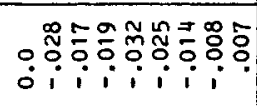 \\
\hline \begin{tabular}{l|l}
0 \\
\end{tabular} & 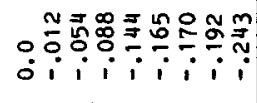 \\
\hline 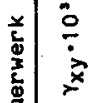 & 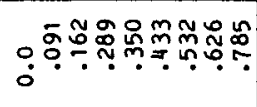 \\
\hline 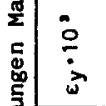 & 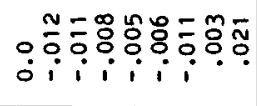 \\
\hline 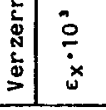 & 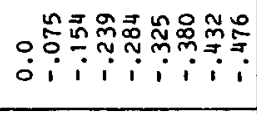 \\
\hline 范鹤 & 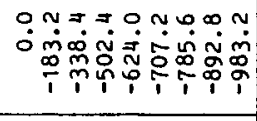 \\
\hline 要 & 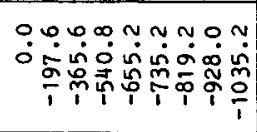 \\
\hline 岁泀言 & $-\sim m a n$ \\
\hline
\end{tabular}

\begin{tabular}{|c|c|c|}
\hline \multirow{2}{*}{ 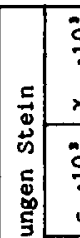 } & \begin{tabular}{|l|}
$\vdots$ \\
$\frac{1}{x}$ \\
\end{tabular} & 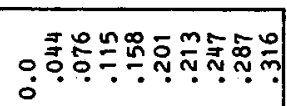 \\
\hline & \begin{tabular}{|l|}
$\dot{0}$ \\
$\dot{3}$
\end{tabular} & 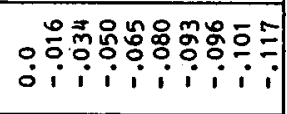 \\
\hline 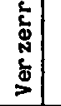 & $\begin{array}{l}\vdots \\
\vdots \\
\dot{x}\end{array}$ & 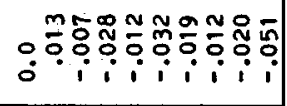 \\
\hline \begin{tabular}{|l|}
$x$ \\
0 \\
0 \\
3 \\
\end{tabular} & \begin{tabular}{|l|}
$\vdots$ \\
$\dot{x}$ \\
$\dot{x}$
\end{tabular} & 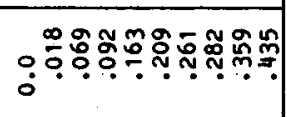 \\
\hline 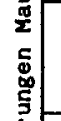 & $\begin{array}{l}0 \\
0 \\
0 \\
0\end{array}$ & 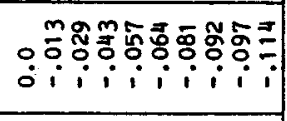 \\
\hline & $\frac{\dot{0}}{\dot{a}}$ & 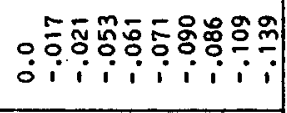 \\
\hline & $\sqrt{8}$ & 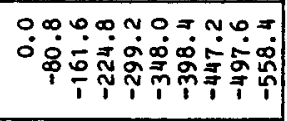 \\
\hline & 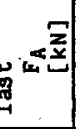 & 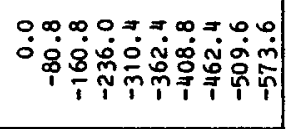 \\
\hline 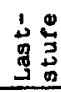 & & $-N M$ \\
\hline
\end{tabular}

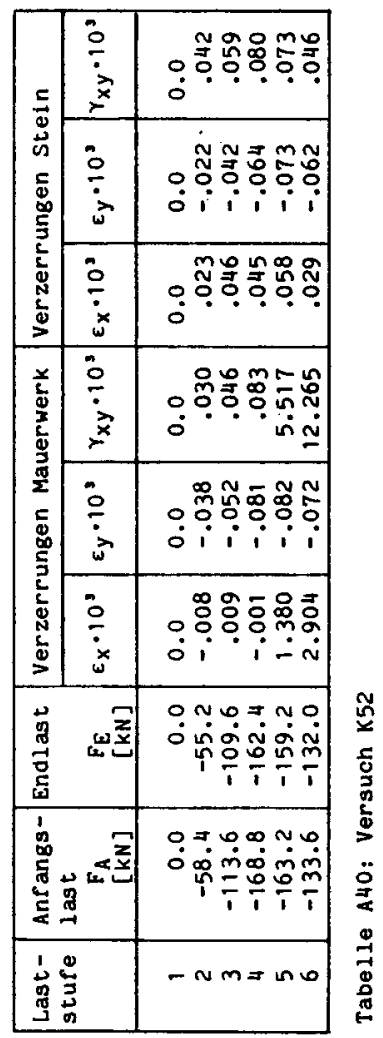

\begin{tabular}{|c|c|}
\hline 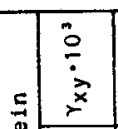 & 疋 \\
\hline 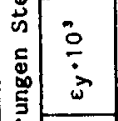 & 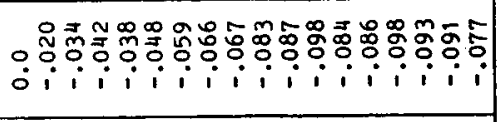 \\
\hline 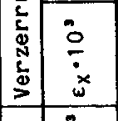 & 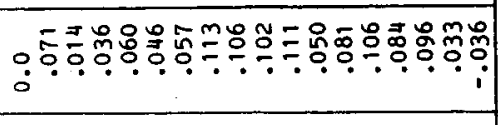 \\
\hline 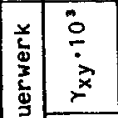 & 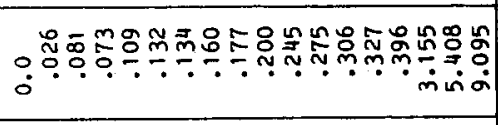 \\
\hline \begin{tabular}{|l|l|}
$\frac{\pi}{2}$ & 0 \\
& 0 \\
0 & 0 \\
0 & 0 \\
$\vdots$ & 0 \\
\end{tabular} & 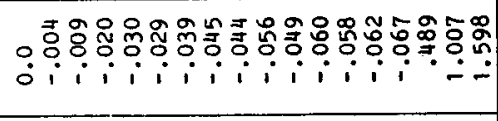 \\
\hline 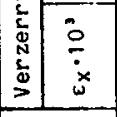 & 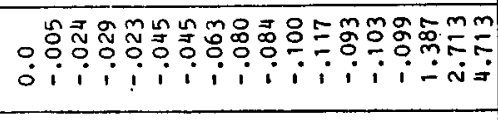 \\
\hline 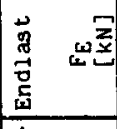 & 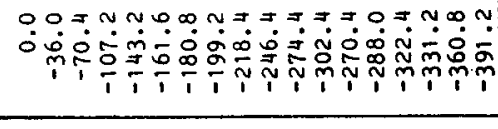 \\
\hline 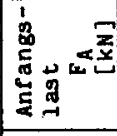 & 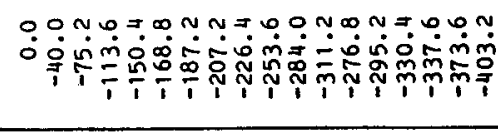 \\
\hline 道离 & \\
\hline
\end{tabular}
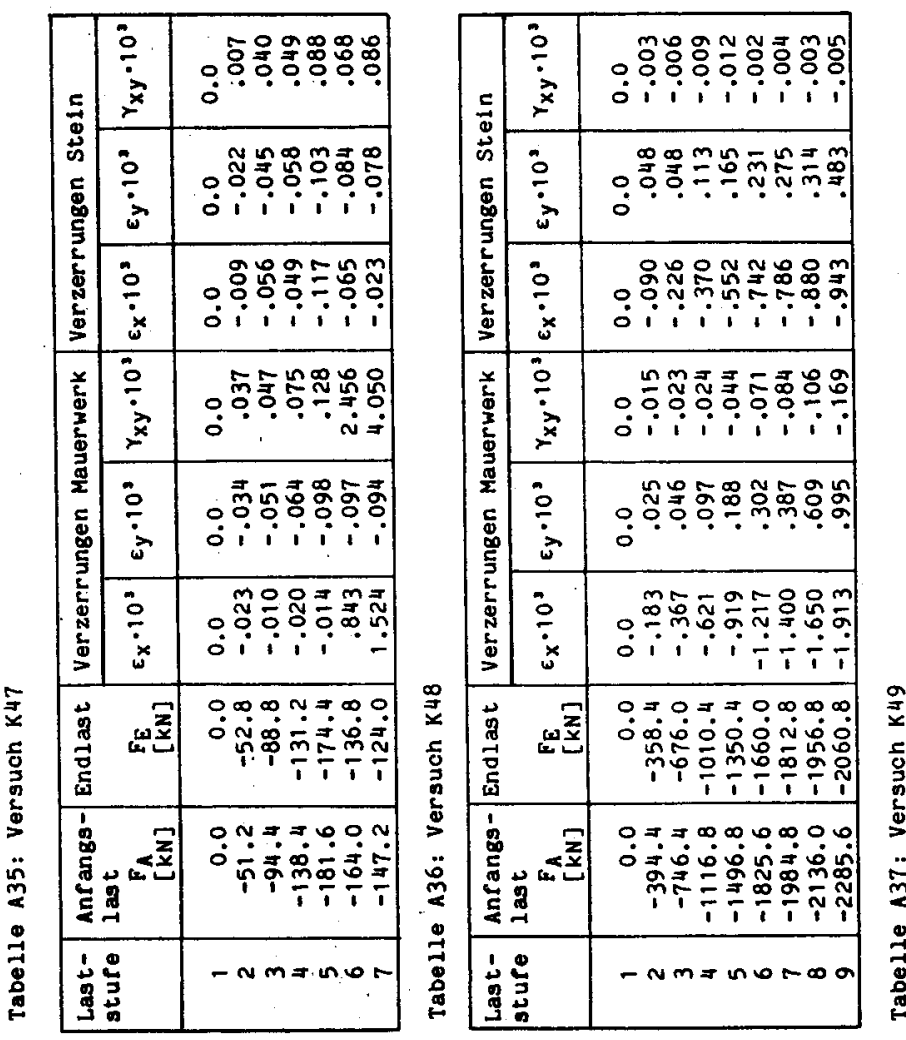


\begin{tabular}{|c|c|c|}
\hline \multirow{3}{*}{ 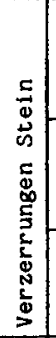 } & 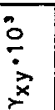 & 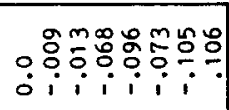 \\
\hline & $\frac{0}{i}$ & 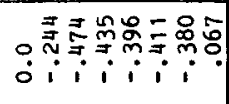 \\
\hline & $\frac{0}{i x}$ & 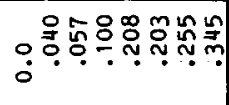 \\
\hline \multirow{3}{*}{ 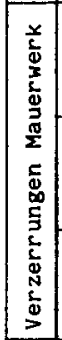 } & $\stackrel{n}{0}$ & 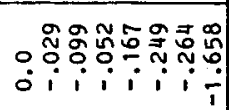 \\
\hline & $\stackrel{0}{0}$ & 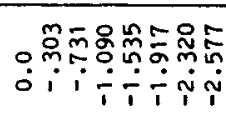 \\
\hline & $\frac{0}{i x}$ & بِ \\
\hline 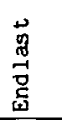 & $\underset{7}{\Xi}$ & 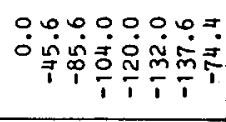 \\
\hline 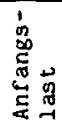 & $\therefore \sqrt[\square]{z}$ & 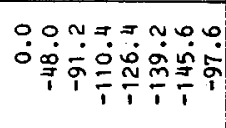 \\
\hline 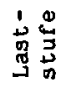 & & $-\sim m=$ \\
\hline
\end{tabular}

\begin{tabular}{|c|c|c|}
\hline \multirow{3}{*}{ 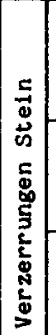 } & $\stackrel{0}{\grave{0}}$ & 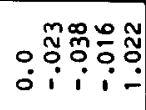 \\
\hline & $\frac{0}{j}$ & 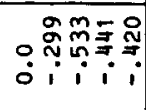 \\
\hline & $\frac{0}{x}$ & 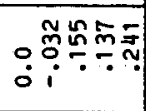 \\
\hline \multirow{3}{*}{ 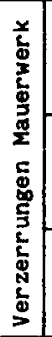 } & $\frac{i}{\dot{x}}$ & 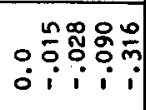 \\
\hline & $\frac{\dot{0}}{\dot{\omega}}$ & 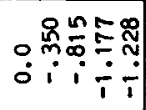 \\
\hline & $\frac{0}{\dot{x}}$ & 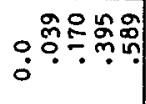 \\
\hline & 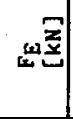 & 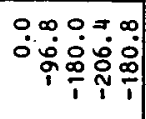 \\
\hline 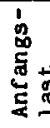 & 吾 & 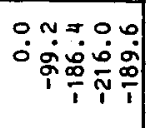 \\
\hline 字: & & $-n$ \\
\hline
\end{tabular}

\begin{tabular}{|c|c|c|}
\hline \multirow{3}{*}{ 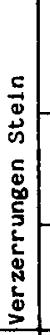 } & 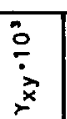 & 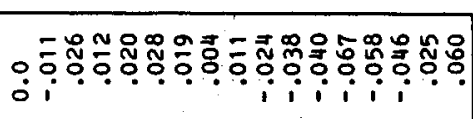 \\
\hline & $\frac{0}{i}$ & 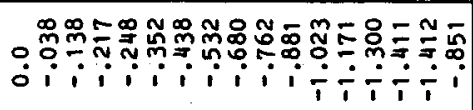 \\
\hline & $\stackrel{n}{i}$ & 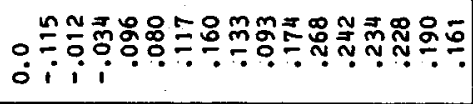 \\
\hline \multirow{3}{*}{ 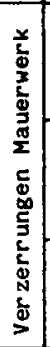 } & $\frac{0}{0}$ & 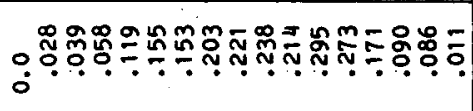 \\
\hline & $\frac{i}{i}$ & 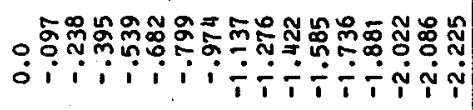 \\
\hline & $\stackrel{0}{0}$ & 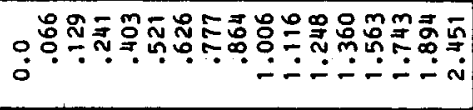 \\
\hline & 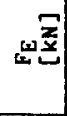 & 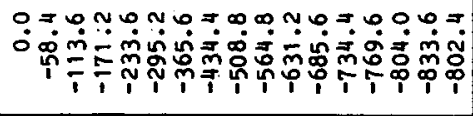 \\
\hline & $+\infty$ & 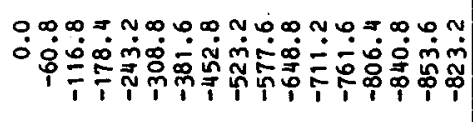 \\
\hline 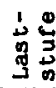 & & - Nman \\
\hline
\end{tabular}

\begin{tabular}{|c|c|c|}
\hline \multirow{3}{*}{ 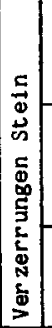 } & \begin{tabular}{l|}
0 \\
0 \\
$\dot{0}$ \\
$x$
\end{tabular} & 웅 \\
\hline & $\frac{0}{0}$ & 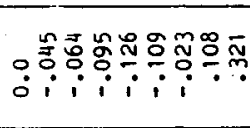 \\
\hline & 立 & 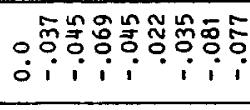 \\
\hline \multirow{3}{*}{ 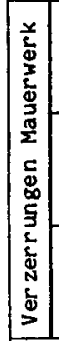 } & 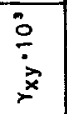 & 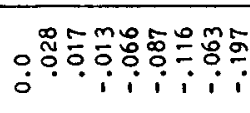 \\
\hline & $\stackrel{n}{\circ}$ & 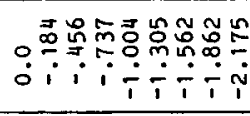 \\
\hline & $\frac{0}{\dot{x}}$ & 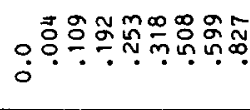 \\
\hline & $\underbrace{\bar{z}}_{\operatorname{ten}}$ & 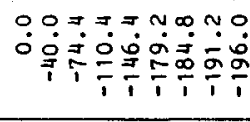 \\
\hline 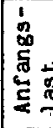 & $=\frac{7}{3}$ & ب \\
\hline 岁 & & $-n$ \\
\hline
\end{tabular}

\begin{tabular}{|c|c|c|}
\hline \multirow{3}{*}{ 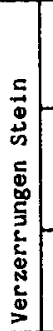 } & $\begin{array}{l}0 \\
0 \\
\vdots \bar{x} \\
x\end{array}$ & 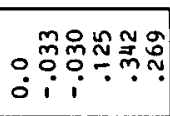 \\
\hline & $\frac{\overline{0}}{\dot{\sigma}}$ & 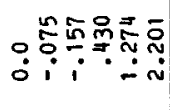 \\
\hline & $\stackrel{0}{0}$ & 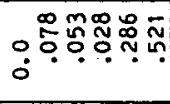 \\
\hline \multirow{3}{*}{ 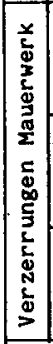 } & $\begin{array}{l}0 \\
0 \\
0 \\
7\end{array}$ & 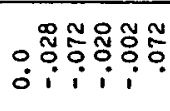 \\
\hline & $\frac{0}{0}$ & 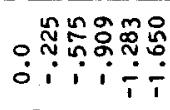 \\
\hline & $\stackrel{0}{\dot{0}}$ & 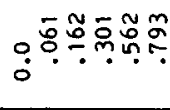 \\
\hline$\stackrel{\vec{a}}{\vec{g}}$ & ב & أْ \\
\hline 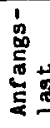 & as & نِ \\
\hline 思 & & $-n$ \\
\hline
\end{tabular}

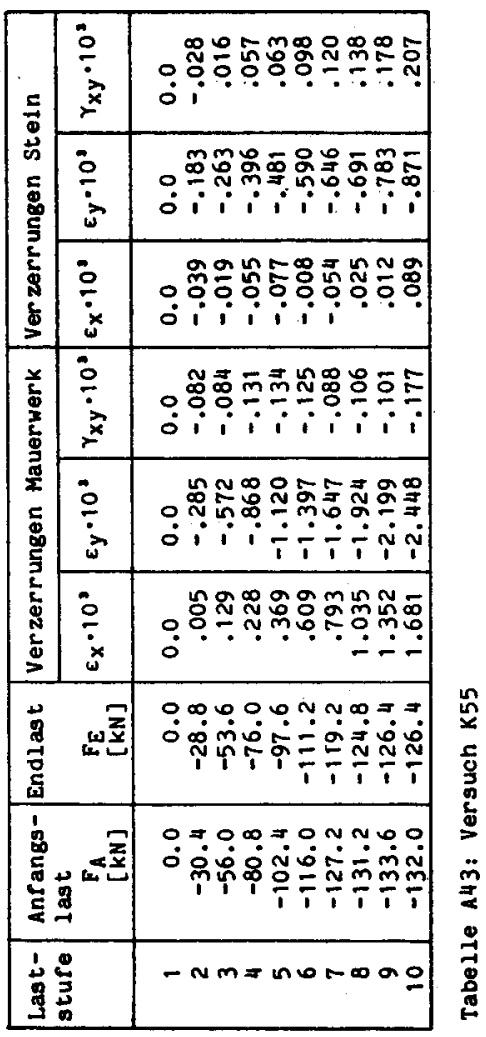




\begin{tabular}{|c|c|}
\hline 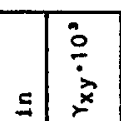 & 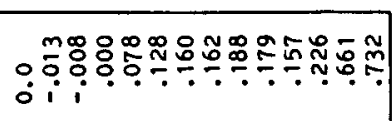 \\
\hline 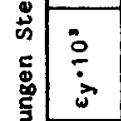 & 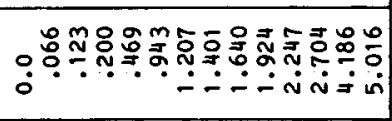 \\
\hline 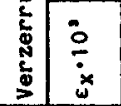 & 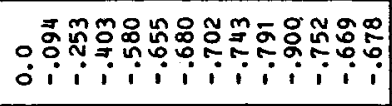 \\
\hline 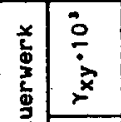 & 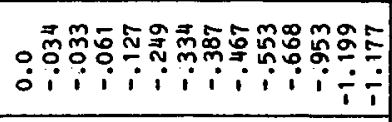 \\
\hline 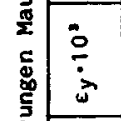 & 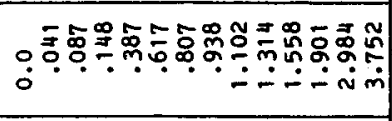 \\
\hline 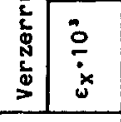 & 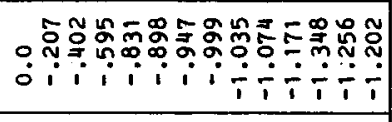 \\
\hline 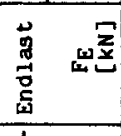 & 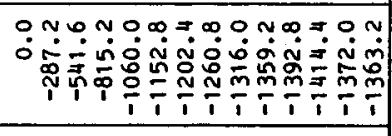 \\
\hline 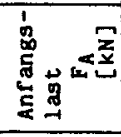 & 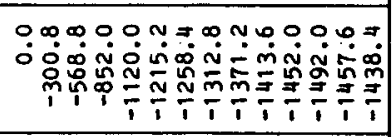 \\
\hline 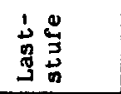 & $m z$ \\
\hline
\end{tabular}

\begin{tabular}{|c|c|}
\hline 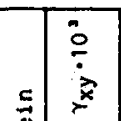 & 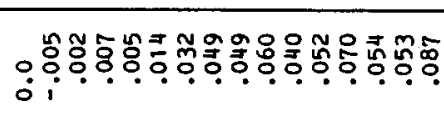 \\
\hline 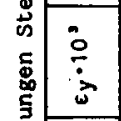 & 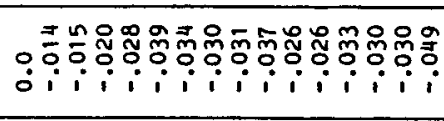 \\
\hline 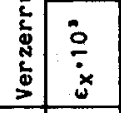 & 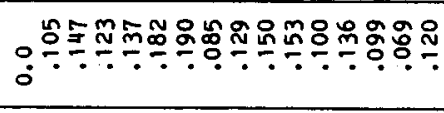 \\
\hline 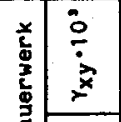 & 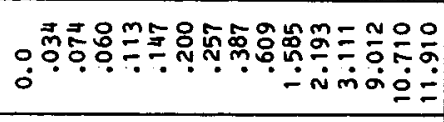 \\
\hline 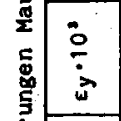 & 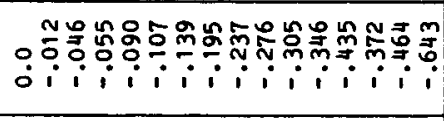 \\
\hline 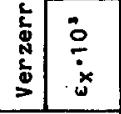 & 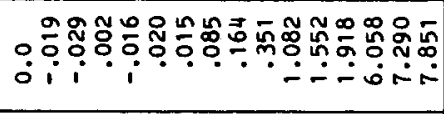 \\
\hline 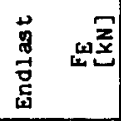 & 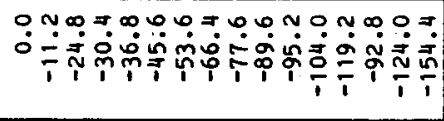 \\
\hline 要 & 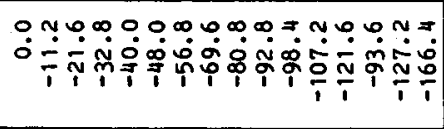 \\
\hline 点总 & $-N$ \\
\hline
\end{tabular}

\begin{tabular}{|c|c|c|}
\hline & 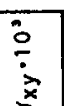 & 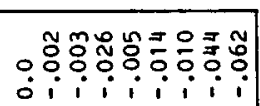 \\
\hline 离 & $\frac{1}{0}$ & 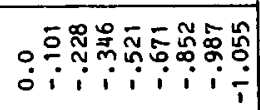 \\
\hline 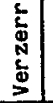 & $\frac{0}{\dot{x}}$ & 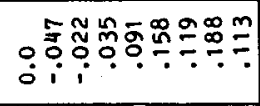 \\
\hline 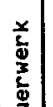 & $\frac{0}{0}$ & 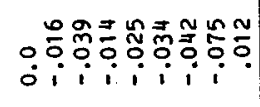 \\
\hline 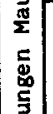 & $\frac{1}{3}$ & 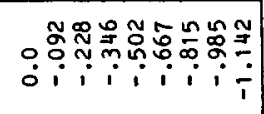 \\
\hline \begin{tabular}{|c|} 
\\
$\frac{1}{2}$ \\
0 \\
0 \\
\\
\\
\end{tabular} & $\begin{array}{c}\dot{0} \\
\dot{x} \\
\end{array}$ & 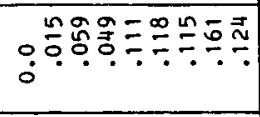 \\
\hline 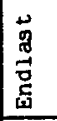 & $\omega_{0}^{\infty \bar{z}}$ & 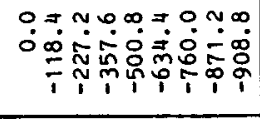 \\
\hline 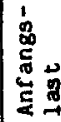 & & بْ \\
\hline 离总 & & $-N m=\ln 0 \times \infty a$ \\
\hline
\end{tabular}
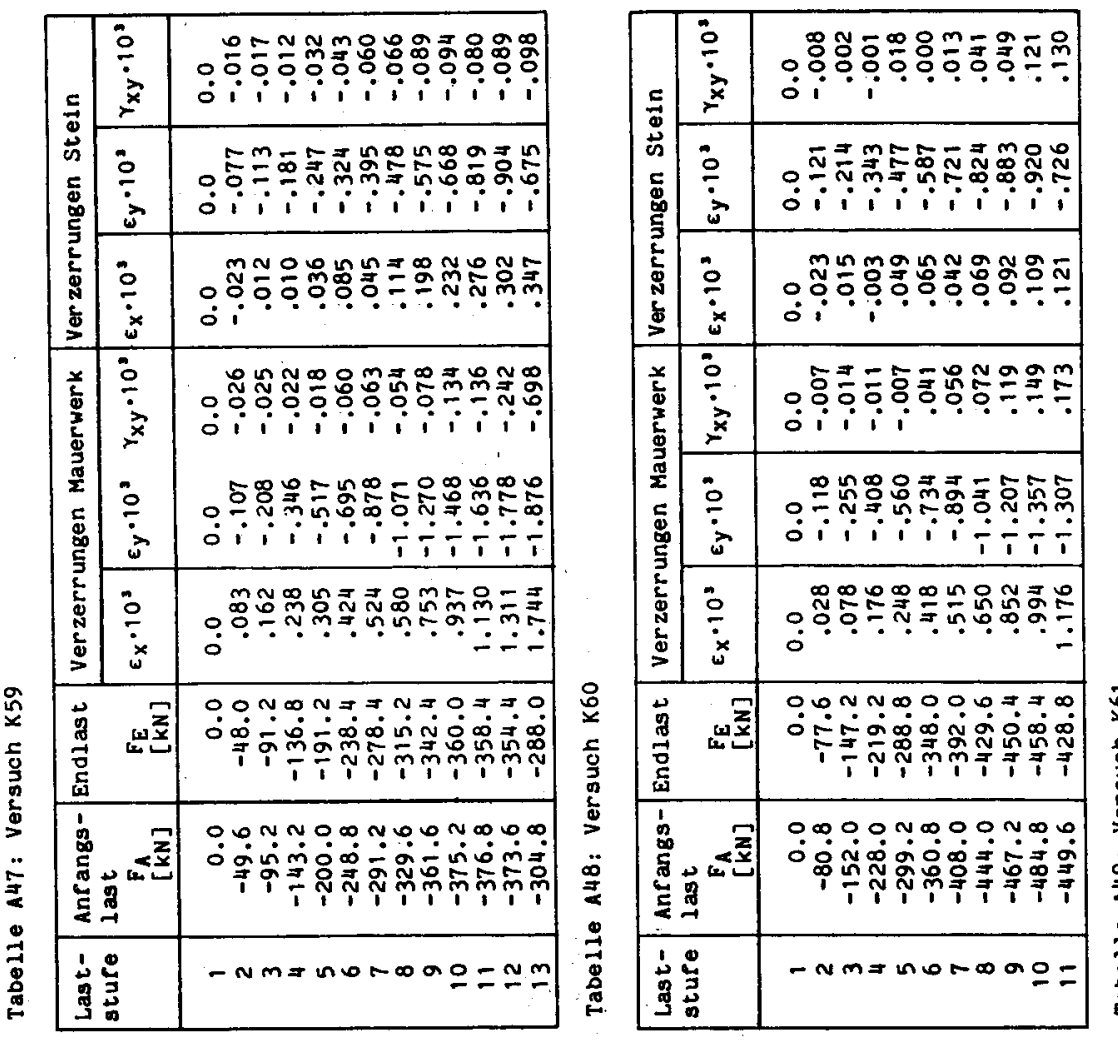
ANHANG B

\section{RILEM-Versuche}

Als Ergänzung zum Versuchsprogramm wurden $15 \mathrm{~K}$ leinversuche mit RILEM-Körpern durchgeführt (Tabelle B1). Die RILEM-Richtlinie [12] schreibt für Versuchskörper aus Mauerwerk vor, dass sie mindestens zwei Steine breit und fünf Steinlagen hoch sein müssen. Das Verhältnis Höhe/Wandstärke (Schlankheit) soll zwischen 3 und 5 , das Verhältnis Höhe/Breite sol1 grösser als 1 sein.

Die Versuchskörper sind im Bild B1 dargestellt. Die Breite betrug 2.5 Steine, wodurch für den ganzen Körper die Anzahl Stossfugen pro Steinlage gleich war. Die vorgeschriebene Schlankheit konnte nicht überall eingehalten werden.

Die RILEM-Versuche wurden prinzipiell gleich wie die übrigen Versuche durchgeführt. Die Messtechnik entsprach derjenigen der Versuche mit den $90^{\circ}$-Körpern. Die Versuche R1 bis R3 wurden ohne Deformationsmessungen vorgenommen. Mit diesen drei Versuchen mit kontinuierlicher Belastung und einer Verformungsgeschwindigkeit nach DIN 1053 von $\dot{\epsilon}_{x}=0.0001$ Min $^{-1}$ wurde abgeklärt, welchen Einfluss der Belastungsvorgang hat. Zum Vergleich wurden drei identische RILEM-Körper nach dem im Abschnitt 3.2 beschriebenen Verfahren belastet (R4 bis R6). Die Resultate sind in den Tabellen B2 bis B13 dargeste11t. Die Uebereinstimmung der RILEMVersuche mit den Versuchen $K 13$ bis $K 63$ ist gut. Es war festzustellen, dass aufgrund der geringen Abmessungen der Versuchskörper starke Schwankungen der Festigkeitseigenschaften der Steine in einer Produktionscharge zu bedeutenden Streuungen der Werte für den Mauerwerkskörper führen können. Deshalb sollten pro Stein-Mörtel-Kombination mindestens drei Versuche durchgeführt werden, wie dies die RILEM-Richtlinie auch vorschreibt.

Der Versuch R9 mit verlängertem Mörtel konnte nicht durchgeführt werden, weil zu wenig Steine vorhanden waren. Mit dem Stein der Serie 2 wurde jedoch ein RILEMKörper mit reinem Kalkmörtel hergestellt (R15). Diese extreme Kombination eines in bezug auf Festigkeit schlechten Mörtels mit einem eher guten Stein erbrachte beim Vergleich desselben Körpers mit Zementmörtel einen starken Abfall der Mauerwerksfestigkeit in x-Richtung von ca. 30\%. Der Versuch R14, mit dem durch Verwendung eines Gussmörtels eine Erhöhung der Mauerwerksfestigkeit erreicht 
werden sollte, erbrachte die aufgrund der Versuche mit vorfabriziertem Mauerwerk erhofften Resultate nicht. Es ist anzunehmen, dass die Herstellungsart eine Rolle spielt, weil hier der Mörtel nicht gegossen werden konnte und deshalb mit der Kelle gearbeitet wurde, was einen steifplastischen Mörtel voraussetzt.

\section{Abscherversuche}

Für die Bestimmung der Fugenkennwerte wurden verschiedene Möglichkeiten für Kleinversuche untersucht. Es zeigte sich, dass insbesondere die Forderung nach einer unbehinderten Verformbarkeit des Probekörpers entscheidend in bezug auf Aussagekraft der Prüfung ist. Da die lokale Spannungsverteilung im Bereich der Lagerfuge kaum erfassbar ist, kann ein Kleinversuch nicht von diesem Gesichtspunkt aus beurteilt werden. Die Herstellung der Proben und die Durchführung solcher Kleinversuche sollte mit möglichst kleinem Aufwand geschehen, und die Anzahl Proben muss genügend gross sein, um die Streuung auszugleichen.

Aufgrund all dieser Erwägungen erschien die Haftscherprüfung DIN 18555/5 am geeignetsten. Das Prüf- und Forschungsinstitut $(P+F)$ der Ziegelindustrie in Sursee hat dieses Prüfverfahren weiterentwickelt. Im Bild B3 ist die Prüfeinrichtung schematisch dargestel1t. Die Belastungseinrichtung erzeugt eine Kraftresultierende, die schräg durch den Probekörper abgetragen wird. Dadurch erhält die Lagerfuge eine Normalkraft sowie eine Scherkraft. Durch die Abmessungen der Probe kann die Neigung der Resultierenden und damit die Beanspruchung der Fuge beeinflusst werden. Es wurde darauf geachtet, dass die Proben in der HerstelTungsart den entsprechenden Ausschnitten aus Mauerwerkswänden entsprachen. Die Resultate dieser Abscherprüfungen nach $D I N / P+F$ sind im $B i l d ~ B 3$ dargestellt. Es konnten nur Prüfungen mit dem Stein der Serie 2 durchgeführt werden.

Bei einer anderen Abscherprüfung, die erprobt wurde, war die Probe von einem Betonkörper umgeben. Die Probe war zur Belastungsrichtung geneigt, wodurch eine zur Lagerfuge geneigte Kraftresultierende erzeugt wurde. Diese Prüfung war ebenfalls gut, aber wesentlich aufwendiger. Die Resultate sind im Bild B3 dargestellt. Das Bild B2 zeigt einen Vergleich der Resultate der beiden Abscherprïfungen mit den Resultaten der Versuche K18 und K63 der Serie 2. 
Modulsteine :

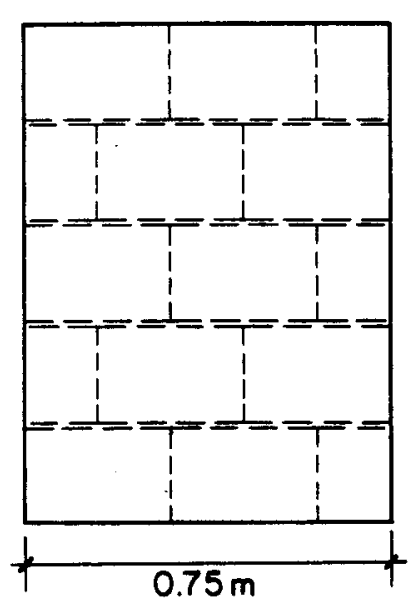

Sichtstein, Calmo:

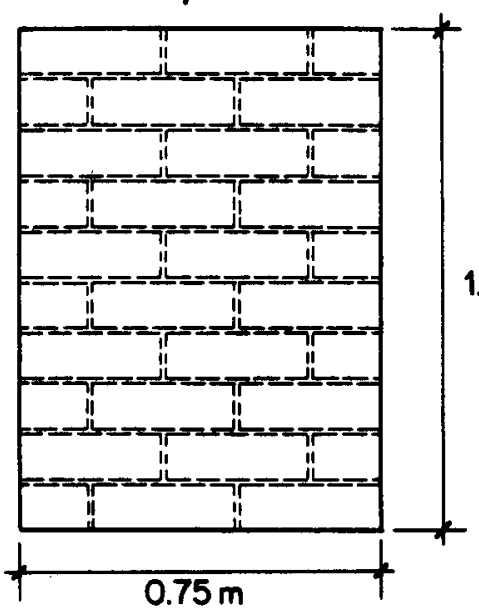

Normalsteine :

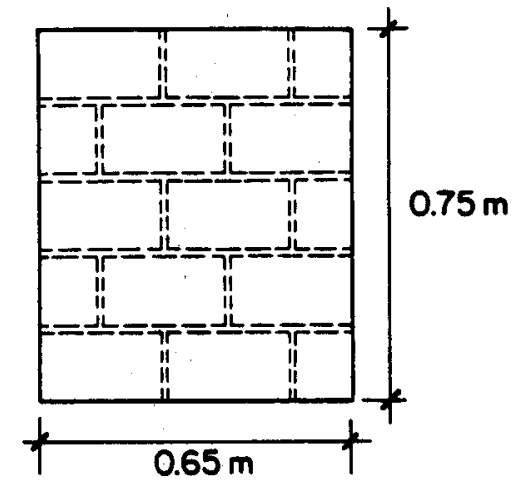

Bild B 1: Rilem-Versuchskörper

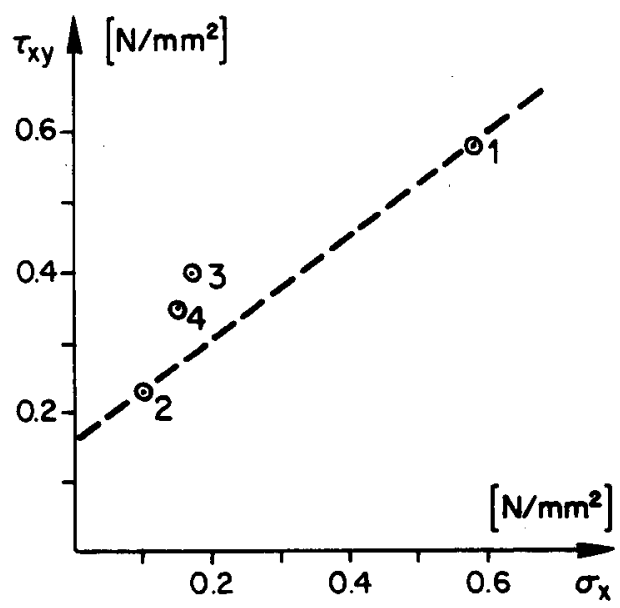

Legende :

1 Versuch $\mathrm{K} 18$

2 Versuch $k 63$

3 Scherversuch DIN/P+F

4 Scherversuch mit Betonummontelung

Bild B2: Vergleich der Scherversuche mit den Versuchen K 18 und K63 


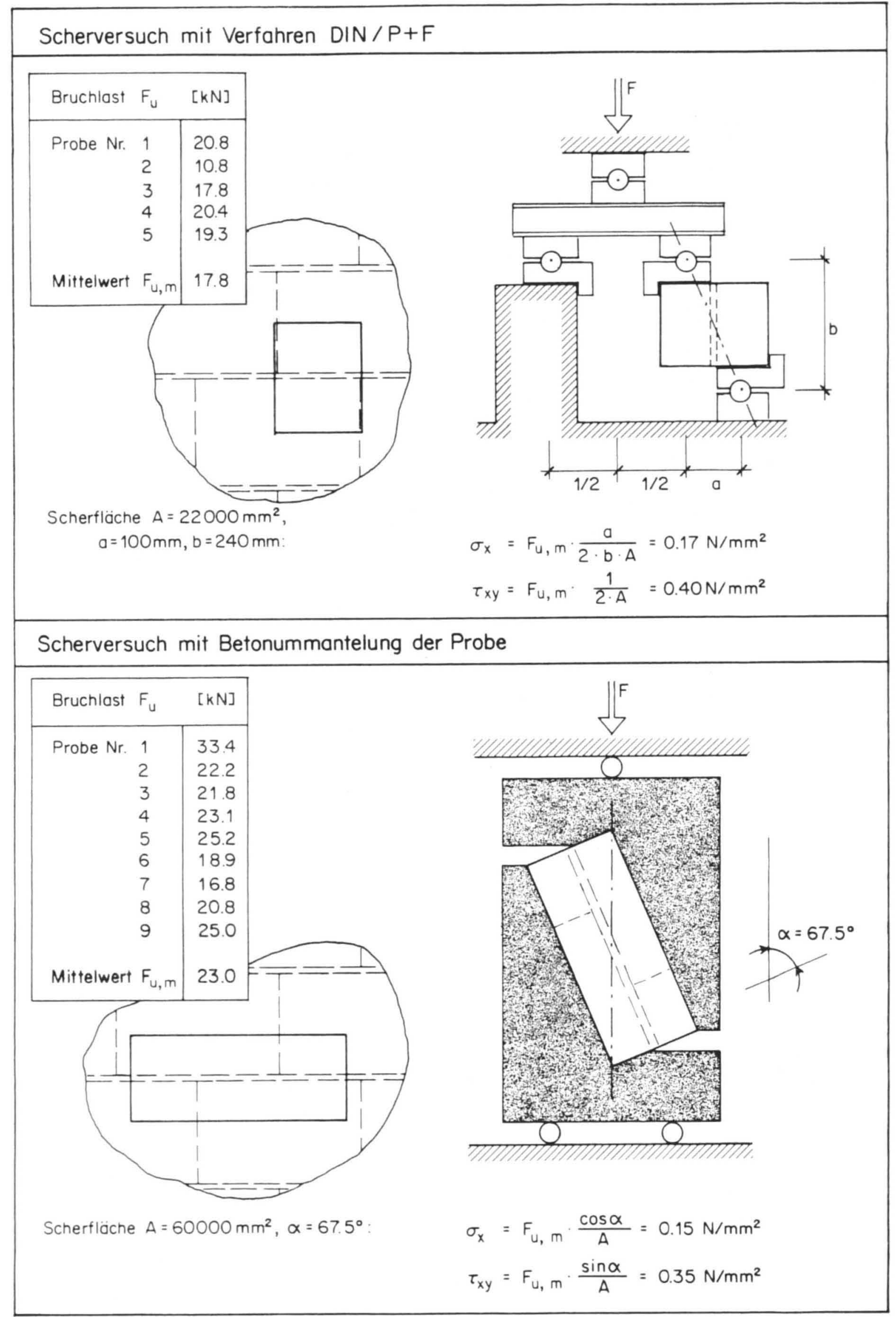

Bild B3: Scherversuche 


\begin{tabular}{|c|c|c|c|c|c|}
\hline \multirow[t]{2}{*}{ Versuch } & \multicolumn{2}{|r|}{ Stein } & \multirow[t]{2}{*}{ wrtel } & \multirow{2}{*}{$\begin{array}{l}\text { Vers :- } \\
\text { serie }\end{array}$} & \multirow[t]{2}{*}{ Bemerkungen } \\
\hline & Prov. & Bezel chnung & & & \\
\hline $\begin{array}{l}\mathbf{R} 1 \\
\mathbf{R 2} \\
\mathbf{R} 3\end{array}$ & $\begin{array}{l}\text { B } \\
\text { B } \\
\text { B }\end{array}$ & B $15 \mathrm{M}$ & Zement & $\begin{array}{l}2 \\
2 \\
2\end{array}$ & $\left\{\begin{array}{l}\text { kont inuir lich } \\
\text { be las tet, onne } \\
\text { Deformetermessung }\end{array}\right.$ \\
\hline $\begin{array}{l}\text { R4 } \\
\text { R5 } \\
\text { R6 } \\
\text { R7 } \\
\text { R8 } \\
\text { R10 } \\
\text { R11 } \\
\text { R12 } \\
\text { R13 }\end{array}$ & $\begin{array}{l}B \\
B \\
B \\
C \\
D \\
G \\
H \\
L \\
M\end{array}$ & $\begin{array}{ll}\text { B } & 15 M \\
& \\
\text { B } & 15 M \\
\text { B } & 15 \\
\text { B } & 17.5 \text { M Calmo } \\
\text { B } & 14 / 9 \text { M S } \\
\text { K } & 15 \\
\text { K } & 15\end{array}$ & $\begin{array}{l}\text { Zement } \\
\text { Zement } \\
\text { Zement } \\
\text { Zement } \\
\text { Zement } \\
\text { Zement } \\
\text { Zement }\end{array}$ & $\begin{array}{r}2 \\
2 \\
2 \\
3 \\
4 \\
7 \\
8 \\
11 \\
12\end{array}$ & $\begin{array}{l}\text { Wiederholung } \\
\text { zur Beobachtung } \\
\text { der Streuung }\end{array}$ \\
\hline $\begin{array}{l}\text { R14 } \\
\text { R15 }\end{array}$ & $\begin{array}{l}\text { B } \\
\text { B }\end{array}$ & $\begin{array}{lll}\text { B } & 15 & M \\
\text { B } & 15 & M\end{array}$ & $\begin{array}{l}\text { Gus sm. } \\
\text { HK } 300\end{array}$ & & $\begin{array}{l}\text { Zusat zversuch } \\
\text { Zusatzversuch }\end{array}$ \\
\hline
\end{tabular}

Tabelle B1: RILEM-Versuchs program

\begin{tabular}{|c|c|c|c|c|c|c|}
\hline \multirow{2}{*}{$\begin{array}{l}\text { Vers.- } \\
\text { serie }\end{array}$} & \multirow[t]{2}{*}{ Vorsuoh } & \multicolumn{2}{|c|}{ Bruchlast $[\mathrm{kN}]$} & \multicolumn{2}{|c|}{$E_{x}\left[N / \mathrm{mm}^{2}\right]$} & \multirow{2}{*}{$\begin{array}{c}\text { Mauerwerks- } \\
\text { festigkelt } \\
\mathrm{r}_{\mathrm{Mx}}-\mathrm{F}_{\mathrm{u}, 1} / \mathrm{A} \\
{\left[\mathrm{N} / \mathrm{mm}^{2}\right]}\end{array}$} \\
\hline & & $\begin{array}{c}\text { kur zze } 1 \text { t ige } \\
\text { Lastspitze } \\
F_{u, k}\end{array}$ & $\begin{array}{l}\text { Langze1t- } \\
\text { wert } \\
\qquad F_{u, 1}\end{array}$ & Mauerwerk & Stein & \\
\hline 2 & $\begin{array}{l}\text { R1 } \\
\text { R2 } \\
\text { R3 }\end{array}$ & $\begin{array}{l}-1160 \\
-1155 \\
-1043\end{array}$ & & & & \\
\hline $\begin{array}{r}3 \\
4 \\
7 \\
8 \\
11 \\
12\end{array}$ & $\begin{array}{l}\text { R4 } \\
R 5 \\
R 6 \\
R 7 \\
\text { R8 } \\
\text { R10 } \\
\text { R11 } \\
\text { R12 } \\
\text { R13 }\end{array}$ & $\begin{array}{r}-1095 \\
-780 \\
-1016 \\
-1240 \\
-610 \\
-1265 \\
-1935 \\
-1137 \\
-1335\end{array}$ & $\begin{array}{r}-1000 \\
-706 \\
-895 \\
-i 126 \\
-560 \\
-994 \\
-1735 \\
-1008 \\
-1190\end{array}$ & $\begin{array}{r}5800 \\
5150 \\
6350 \\
7900 \\
2800 \\
3100 \\
13750 \\
8000 \\
7900\end{array}$ & $\begin{array}{r}10500 \\
11350 \\
10450 \\
11350 \\
4800 \\
19900 \\
18150 \\
13750 \\
12550\end{array}$ & $\begin{array}{r}8.89 \\
6.28 \\
7.96 \\
10.01 \\
5.74 \\
7.57 \\
16.52 \\
10.69 \\
12.63\end{array}$ \\
\hline & $\begin{array}{l}\text { R14 } \\
\text { R15 }\end{array}$ & $\begin{array}{r}-1145 \\
-732\end{array}$ & $\begin{array}{r}-1072 \\
-673\end{array}$ & $\begin{array}{l}5700 \\
4200\end{array}$ & $\begin{array}{l}8250 \\
7700\end{array}$ & \\
\hline
\end{tabular}

Tabelle B2: Resultate der Rilemversuche

\begin{tabular}{|c|c|c|c|c|c|c|c|c|}
\hline \multirow{2}{*}{$\begin{array}{l}\text { Last- } \\
\text { stufe }\end{array}$} & \multirow{2}{*}{$\begin{array}{c}\text { Anf angs - } \\
\text { last } \\
E_{\mathrm{A}} \\
{[\mathrm{kN}]}\end{array}$} & \multirow{2}{*}{$\begin{array}{c}\text { Endlast } \\
F_{E} \\
{[\mathrm{kN}]}\end{array}$} & \multicolumn{3}{|c|}{ Verzerrungen Mauerwerk } & \multicolumn{3}{|c|}{ Verzerrungen Stein } \\
\hline & & & $\varepsilon_{x} \cdot 10^{3}$ & $\varepsilon_{y} \cdot 10^{3}$ & $Y_{x y} \cdot 10^{3}$ & $\varepsilon_{x} \cdot 10^{\prime}$ & $\varepsilon_{y} \cdot 10^{3}$ & $\gamma_{x y} \cdot 10^{\prime}$ \\
\hline $\begin{array}{l}1 \\
2 \\
3 \\
4 \\
5 \\
6\end{array}$ & $\begin{array}{r}0.0 \\
-270.4 \\
-545.6 \\
-833.6 \\
-974.4 \\
-1020.0\end{array}$ & $\begin{array}{r}0.0 \\
-266.4 \\
-537.6 \\
-822.4 \\
-952.0 \\
-1000.0\end{array}$ & $\begin{array}{l}0.0 \\
-.340 \\
-.774 \\
-1.261 \\
-1.465 \\
-1.764\end{array}$ & $\begin{array}{l}0.0 \\
.021 \\
.063 \\
.159 \\
.230 \\
.842\end{array}$ & $\begin{array}{l}0.0 \\
.010 \\
.057 \\
.080 \\
.092 \\
-.116\end{array}$ & $\begin{array}{l}0.0 \\
-.204 \\
-.419 \\
-.632 \\
-.807 \\
-.875\end{array}$ & $\begin{array}{l}0.0 \\
.065 \\
.114 \\
.210 \\
.257 \\
.317\end{array}$ & $\begin{array}{l}0.0 \\
.009 \\
.042 \\
.023 \\
-.029 \\
-.0303\end{array}$ \\
\hline
\end{tabular}

Tabelle B3: RIlemversuch R4

\begin{tabular}{|c|c|c|c|c|c|c|c|c|}
\hline \multirow{2}{*}{$\begin{array}{l}\text { Last- } \\
\text { stufe }\end{array}$} & \multirow{2}{*}{$\begin{array}{c}\text { Anfangs - } \\
\text { last } \\
F_{\mathrm{A}} \\
{[\mathrm{kN}]}\end{array}$} & \multirow{2}{*}{$\begin{array}{c}\text { Endlast } \\
F_{E} \\
{[k N]}\end{array}$} & \multicolumn{3}{|c|}{ Verzerrungen Mauerwerk } & \multicolumn{3}{|c|}{ Verzerrungen Stein } \\
\hline & & & $\varepsilon_{x} \cdot 10^{3}$ & $\varepsilon_{y} \cdot 10^{\prime}$ & $Y_{x y} \cdot 10^{\prime}$ & $\varepsilon_{x} \cdot 10^{3}$ & $E y \cdot 10^{3}$ & $Y_{X y} \cdot 10^{3}$ \\
\hline $\begin{array}{l}1 \\
2 \\
3 \\
4 \\
5 \\
6\end{array}$ & $\begin{array}{r}0.0 \\
-271.2 \\
-556.0 \\
-676.0 \\
-723.2 \\
-716.8\end{array}$ & $\begin{array}{r}0.0 \\
-268.0 \\
-544.8 \\
-660.0 \\
-706.4 \\
-636.8\end{array}$ & $\begin{array}{l}0.0 \\
-.393 \\
-.898 \\
-1.144 \\
-1.465 \\
-1.775\end{array}$ & $\begin{array}{l}0.0 \\
.066 \\
.295 \\
.485 \\
.960 \\
1.330\end{array}$ & $\begin{array}{l}0.0 \\
-.018 \\
-.094 \\
-.168 \\
-.184 \\
.019\end{array}$ & $\begin{array}{l}0.0 \\
-.250 \\
-.476 \\
-.517 \\
-.763 \\
-.621\end{array}$ & $\begin{array}{l}0.0 \\
.101 \\
.583 \\
.945 \\
1.295 \\
1.304\end{array}$ & $\begin{array}{l}0.0 \\
.023 \\
.164 \\
.219 \\
.005 \\
.811\end{array}$ \\
\hline
\end{tabular}

Tabelle B4: Rilemversuch A5 


\begin{tabular}{|c|c|c|c|c|c|c|c|c|}
\hline \multirow{2}{*}{$\begin{array}{l}\text { Last- } \\
\text { stufe }\end{array}$} & \multirow{2}{*}{$\begin{array}{c}\text { Anfangs- } \\
\text { last } \\
F_{\mathrm{A}} \\
{[\mathrm{kN}]}\end{array}$} & \multirow{2}{*}{$\begin{array}{c}\text { End last } \\
\\
F_{E} \\
{[\mathrm{kN}]}\end{array}$} & \multicolumn{3}{|c|}{ Verzerrungen Mauerwerk } & \multicolumn{3}{|c|}{ Ver zerrungen Stein } \\
\hline & & & $\varepsilon_{x} \cdot 10^{3}$ & $\varepsilon_{y} \cdot 10^{3}$ & $Y_{x y} \cdot 10^{3}$ & $\varepsilon_{x} \cdot 10^{3}$ & $\varepsilon_{y} \cdot 10^{3}$ & $Y_{x y} \cdot 10^{3}$ \\
\hline $\begin{array}{l}1 \\
2 \\
3 \\
4 \\
5 \\
6 \\
7\end{array}$ & $\begin{array}{r}0.0 \\
-272.0 \\
-540.8 \\
-794.4 \\
-900.0 \\
-934.4 \\
-908.8\end{array}$ & $\begin{array}{r}0.0 \\
-268.0 \\
-532.8 \\
-781.6 \\
-877.6 \\
-895.2 \\
-864.8\end{array}$ & $\begin{array}{l}0.0 \\
-.298 \\
-.671 \\
-1.036 \\
-1.229 \\
-1.410 \\
-1.340\end{array}$ & $\begin{array}{l}0.0 \\
.031 \\
.090 \\
.226 \\
.568 \\
.961 \\
1.341\end{array}$ & $\begin{array}{l}0.0 \\
-.045 \\
-.043 \\
-.022 \\
-.067 \\
-.092 \\
-.154\end{array}$ & $\begin{array}{l}0.0 \\
-.201 \\
-.396 \\
-.656 \\
-.747 \\
-.631 \\
-.482\end{array}$ & $\begin{array}{l}0.0 \\
.090 \\
.156 \\
.237 \\
.487 \\
.666 \\
.860\end{array}$ & $\begin{array}{l}0.0 \\
.013 \\
.056 \\
.099 \\
.281 \\
.484 \\
.6 .12\end{array}$ \\
\hline
\end{tabular}

Tabelle B5: R1lemversuch R6

\begin{tabular}{|c|c|c|c|c|c|c|c|c|}
\hline \multirow{2}{*}{$\begin{array}{l}\text { Last- } \\
\text { sture }\end{array}$} & \multirow{2}{*}{$\begin{array}{c}\text { Anfangs- } \\
\text { last } \\
\bar{F}_{\mathrm{A}} \\
{[\mathrm{kN}]}\end{array}$} & \multirow{2}{*}{ 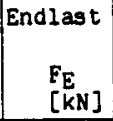 } & \multicolumn{3}{|c|}{ Verzerrungen Mauerwerk } & \multicolumn{3}{|c|}{ Verzerrungen Stein } \\
\hline & & & $\varepsilon_{x} \cdot 10^{3}$ & $\varepsilon_{y} \cdot 10^{3}$ & $\gamma_{x y} \cdot 10^{3}$ & $\varepsilon_{X} \cdot 10^{3}$ & $\varepsilon_{y} \cdot 10^{\prime}$ & $\gamma_{x y} \cdot 10^{3}$ \\
\hline $\begin{array}{r}1 \\
2 \\
3 \\
4 \\
5 \\
6 \\
7 \\
8 \\
9 \\
10\end{array}$ & $\begin{array}{r}0.0 \\
-225.6 \\
-443.2 \\
-662.4 \\
-769.6 \\
-876.8 \\
-984.0 \\
-1054.4 \\
-1121.6 \\
-1183.2\end{array}$ & $\begin{array}{r}0.0 \\
-221.6 \\
-436.8 \\
-653.6 \\
-759.2 \\
-865.6 \\
-969.6 \\
-1032.0 \\
-1103.2 \\
-1125.6\end{array}$ & $\begin{array}{l}0.0 \\
-.166 \\
-.399 \\
-.620 \\
-.797 \\
-.943 \\
-1.088 \\
-1.140 \\
-1.116 \\
-1.119\end{array}$ & $\begin{array}{l}0.0 \\
.028 \\
.053 \\
.081 \\
.150 \\
.183 \\
.254 \\
.431 \\
.566 \\
.958\end{array}$ & $\begin{array}{l}0.0 \\
-.005 \\
.006 \\
.019 \\
.033 \\
.019 \\
-.014 \\
.067 \\
.081 \\
.019\end{array}$ & $\begin{array}{l}0.0 \\
-.200 \\
-.373 \\
-.515 \\
-.594 \\
-.629 \\
-.760 \\
-.712 \\
-.565 \\
-.473\end{array}$ & $\begin{array}{l}0.0 \\
.045 \\
.091 \\
.144 \\
.243 \\
.296 \\
.407 \\
.567 \\
.677 \\
.867\end{array}$ & $\begin{array}{l}0.0 \\
-.007 \\
.005 \\
.035 \\
.017 \\
-.002 \\
.044 \\
.064 \\
.154 \\
.208\end{array}$ \\
\hline
\end{tabular}

Tabelle B6: Rilemversuch R7

\begin{tabular}{|c|c|c|c|c|c|c|c|c|}
\hline \multirow{2}{*}{$\begin{array}{l}\text { Last- } \\
\text { stufe }\end{array}$} & \multirow{2}{*}{$\begin{array}{c}\text { Anf angs- } \\
\text { last } \\
F_{A} \\
{[\mathrm{kN}]}\end{array}$} & \multirow{2}{*}{$\begin{array}{c}\text { Endlast } \\
\mathrm{FE}_{\mathrm{E}} \\
{[\mathrm{kN}]}\end{array}$} & \multicolumn{3}{|c|}{ Verzerrungen Mauerwerk } & \multicolumn{3}{|c|}{ Ver zerrungen Stein } \\
\hline & & & $\varepsilon_{X} \cdot 10^{3}$ & $\varepsilon_{\mathrm{y}} \cdot 10^{3}$ & $\gamma_{x y} \cdot 10^{3}$ & $\varepsilon_{\mathbf{X}} \cdot 10^{3}$ & $\varepsilon_{y} \cdot 10^{3}$ & $\gamma_{x y} \cdot 10^{\prime}$ \\
\hline $\begin{array}{r}1 \\
2 \\
3 \\
4 \\
5 \\
6 \\
7 \\
8 \\
9 \\
10\end{array}$ & $\begin{array}{r}0.0 \\
-116.8 \\
-233.6 \\
-337.6 \\
-395.2 \\
-446.4 \\
-497.6 \\
-533.6 \\
-568.8 \\
-541.6\end{array}$ & $\begin{array}{r}0.0 \\
-116.0 \\
-230.4 \\
-332.0 \\
-388.8 \\
-439.2 \\
-488.8 \\
-523.2 \\
-555.2 \\
-521.6\end{array}$ & $\begin{array}{l}0.0 \\
-.302 \\
-.673 \\
-1.090 \\
-1.370 \\
-1.612 \\
-1.820 \\
-2.059 \\
-2.323 \\
-2.567\end{array}$ & $\begin{array}{l}0.0 \\
.024 \\
.063 \\
.138 \\
.236 \\
.347 \\
.425 \\
.590 \\
.786 \\
.862\end{array}$ & $\begin{array}{l}0.0 \\
.009 \\
.032 \\
.064 \\
.053 \\
.124 \\
.143 \\
.182 \\
.220 \\
.494\end{array}$ & $\begin{array}{l}0.0 \\
-.141 \\
-.381 \\
-.702 \\
-.832 \\
-.942 \\
-.917 \\
-.765 \\
-.732 \\
-.533\end{array}$ & $\begin{array}{l}0.0 \\
.077 \\
.159 \\
.336 \\
.442 \\
.604 \\
.752 \\
.951 \\
1.190 \\
1.061\end{array}$ & $\begin{array}{l}0.0 \\
-.033 \\
-.037 \\
.084 \\
.105 \\
.198 \\
.293 \\
.587 \\
.713 \\
.949\end{array}$ \\
\hline
\end{tabular}

Tabelle B7: R1lemversuch R8

\begin{tabular}{|c|c|c|c|c|c|c|c|c|}
\hline \multirow{2}{*}{$\begin{array}{l}\text { Last- } \\
\text { stufe }\end{array}$} & \multirow{2}{*}{$\begin{array}{c}\text { Anf angs- } \\
\text { last } \\
\mathbf{F}_{\mathbf{A}} \\
{[\mathbf{k N}]}\end{array}$} & \multirow{2}{*}{$\begin{array}{c}\text { Endlast } \\
\mathrm{F}_{\mathrm{E}} \\
{[\mathrm{KN}]}\end{array}$} & \multicolumn{3}{|c|}{ Verzerrungen Mauerwerk } & \multicolumn{3}{|c|}{ Verzer rungen stein } \\
\hline & & & $\varepsilon_{X} \cdot 10^{3}$ & $\varepsilon_{y} \cdot 10^{3}$ & $\gamma_{x y} \cdot 10^{3}$ & $\varepsilon_{X} \cdot 10^{3}$ & $E_{y} \cdot 10^{3}$ & $\gamma_{x y} \cdot 10^{3}$ \\
\hline $\begin{array}{l}1 \\
2 \\
3 \\
4 \\
5 \\
6 \\
7 \\
8\end{array}$ & $\begin{array}{r}0.0 \\
-216.0 \\
-431.2 \\
-639.2 \\
-832.0 \\
-915.2 \\
-970.4 \\
-1026.4\end{array}$ & $\begin{array}{r}0.0 \\
-209.6 \\
-419.2 \\
-621.6 \\
-812.0 \\
-889.6 \\
-943.2 \\
-993.6\end{array}$ & $\begin{array}{l}0.0 \\
-.430 \\
-.946 \\
-1.541 \\
-2.234 \\
-2.633 \\
-2.849 \\
-3.062\end{array}$ & $\begin{array}{l}0.0 \\
.028 \\
.194 \\
.548 \\
1.086 \\
1.349 \\
1.707 \\
1.941\end{array}$ & $\begin{array}{l}0.0 \\
-.043 \\
-.103 \\
-.219 \\
-.394 \\
-.484 \\
-.524 \\
-.494\end{array}$ & $\begin{array}{l}0.0 \\
-.125 \\
-.253 \\
-.238 \\
-.539 \\
-.587 \\
-.567 \\
-.632\end{array}$ & $\begin{array}{l}0.0 \\
.051 \\
.298 \\
.716 \\
1.112 \\
1.342 \\
1.714 \\
2.030\end{array}$ & $\begin{array}{l}0.0 \\
-.044 \\
-.003 \\
-.012 \\
-.174 \\
-.212 \\
-.302 \\
-.434\end{array}$ \\
\hline
\end{tabular}

Tabelle B8: Rilemversuch R10

\begin{tabular}{|c|c|c|c|c|c|c|c|c|}
\hline \multirow{2}{*}{$\begin{array}{l}\text { Last- } \\
\text { stufe }\end{array}$} & \multirow{2}{*}{$\begin{array}{c}\text { Anfangs- } \\
\text { last } \\
\mathrm{F}_{\mathrm{A}} \\
{[\mathrm{kN}]}\end{array}$} & \multirow{2}{*}{$\begin{array}{l}\text { Endlast } \\
\qquad \begin{array}{l}F_{E} \\
{[\mathrm{kN}]}\end{array}\end{array}$} & \multicolumn{3}{|c|}{ Verzerrungen Mauerwerk } & \multicolumn{3}{|c|}{ Verzerrungen stein } \\
\hline & & & $\varepsilon_{x} \cdot 10^{3}$ & $E_{y} \cdot 10^{\prime}$ & $\gamma_{x y} \cdot 10^{3}$ & $E_{x} \cdot 10^{3}$ & $\varepsilon_{y} \cdot 10^{3}$ & $Y_{x y} \cdot 10^{3}$ \\
\hline $\begin{array}{l}1 \\
2 \\
3 \\
4 \\
5 \\
6 \\
7 \\
8\end{array}$ & $\begin{array}{r}0.0 \\
-417.6 \\
-836.8 \\
-1267.2 \\
-1464.0 \\
-1641.6 \\
-1777.6 \\
-1848.0\end{array}$ & $\begin{array}{r}0.0 \\
-411.2 \\
-825.6 \\
-1246.4 \\
-1436.8 \\
-1607.2 \\
-1722.4 \\
-1698.4\end{array}$ & $\begin{array}{l}0.0 \\
-.259 \\
-.520 \\
-.801 \\
-.960 \\
-1.113 \\
-1.305 \\
-1.684\end{array}$ & $\begin{array}{l}0.0 \\
.041 \\
.077 \\
.134 \\
.188 \\
.239 \\
.390 \\
.733\end{array}$ & $\begin{array}{l}0.0 \\
-.018 \\
-.020 \\
-.024 \\
-.003 \\
.005 \\
-.076 \\
-.221\end{array}$ & $\begin{array}{l}0.0 \\
-.206 \\
-.381 \\
-.654 \\
-.750 \\
-.844 \\
-1.020 \\
-1.094\end{array}$ & $\begin{array}{l}0.0 \\
.032 \\
.098 \\
.169 \\
.204 \\
.252 \\
.258 \\
.269\end{array}$ & $\begin{array}{l}0.0 \\
-.009 \\
-.038 \\
-.035 \\
-.007 \\
.041 \\
-.110 \\
-.176\end{array}$ \\
\hline
\end{tabular}

Tabelle B9: Rilemversuch RII 


\begin{tabular}{|c|c|c|c|c|c|c|c|c|}
\hline \multirow{2}{*}{$\begin{array}{l}\text { Last- } \\
\text { stufe }\end{array}$} & \multirow{2}{*}{$\begin{array}{c}\text { Anf angs - } \\
\text { last } \\
\mathrm{F}_{\mathrm{A}} \\
{[\mathrm{kN}]}\end{array}$} & \multirow{2}{*}{$\begin{array}{c}\text { Endlast } \\
F_{E_{1}} \\
{[\mathrm{kN}]}\end{array}$} & \multicolumn{3}{|c|}{ Verzerrungen Mauerwerk } & \multicolumn{3}{|c|}{ Verzerrungen Stein } \\
\hline & & & $\varepsilon_{x} \cdot 10^{3}$ & $\varepsilon_{y} \cdot 10^{3}$ & $\gamma_{x y} \cdot 10^{3}$ & $\varepsilon_{x} \cdot 10^{\prime}$ & $c_{y} \cdot 10^{3}$ & $\gamma_{x y} \cdot 10^{\prime}$ \\
\hline $\begin{array}{l}1 \\
2 \\
3 \\
4 \\
5 \\
6 \\
7 \\
8\end{array}$ & $\begin{array}{r}0.0 \\
-240.0 \\
-480.0 \\
-704.0 \\
-814.4 \\
-908.0 \\
-1002.4 \\
-1054.4\end{array}$ & $\begin{array}{r}0.0 \\
-234.4 \\
-456.8 \\
-668.8 \\
-791.2 \\
-880.8 \\
-968.8 \\
-1005.6\end{array}$ & $\begin{array}{l}0.0 \\
-.330 \\
-.607 \\
-2.117 \\
-2.346 \\
-2.569 \\
-2.838 \\
-3.147\end{array}$ & $\begin{array}{l}0.0 \\
.021 \\
.071 \\
.148 \\
.189 \\
.248 \\
.351 \\
.521\end{array}$ & $\begin{array}{l}0.0 \\
.026 \\
-.002 \\
-.466 \\
-.472 \\
-.445 \\
-.464 \\
-.455\end{array}$ & $\begin{array}{l}0.0 \\
-.195 \\
-.352 \\
-.734 \\
-.868 \\
-1.101 \\
-1.244 \\
-1.415\end{array}$ & $\begin{array}{l}0.0 \\
.033 \\
.086 \\
.191 \\
.232 \\
.295 \\
.431 \\
.673\end{array}$ & $\begin{array}{l}0.0 \\
-.044 \\
-.094 \\
-.063 \\
-.070 \\
-.059 \\
-.043 \\
.064\end{array}$ \\
\hline
\end{tabular}

Tabelle B10: Rilemversuch R12

\begin{tabular}{|c|c|c|c|c|c|c|c|c|}
\hline \multirow{2}{*}{$\begin{array}{l}\text { Last- } \\
\text { stufe }\end{array}$} & \multirow{2}{*}{$\begin{array}{c}\text { Anfangs- } \\
\text { last } \\
\mathbf{F}_{\mathbf{A}} \\
{[\mathbf{k N}]}\end{array}$} & \multirow{2}{*}{$\begin{array}{c}\text { Endlast } \\
F_{E} \\
{[K N]}\end{array}$} & \multicolumn{3}{|c|}{ Verzerrungen Mauerwerk } & \multicolumn{3}{|c|}{ Ver zerrungen Steln } \\
\hline & & & $\epsilon_{x} \cdot 10^{3}$ & $\varepsilon_{y} \cdot 10^{\prime}$ & $\gamma_{x y} \cdot 10^{\prime}$ & $\varepsilon_{x} \cdot 10^{\prime}$ & $\varepsilon_{y} \cdot 10^{\prime}$ & $\gamma_{x y} \cdot 10^{3}$ \\
\hline $\begin{array}{r}1 \\
2 \\
3 \\
4 \\
5 \\
6 \\
7 \\
8 \\
9 \\
10 \\
11 \\
\end{array}$ & $\begin{array}{r}0.0 \\
-262.4 \\
-529.6 \\
-784.0 \\
-900.0 \\
-1006.4 \\
-1096.0 \\
-1180.0 \\
-1239.2 \\
-1230.4 \\
-894.4 \\
\end{array}$ & $\begin{array}{r}0.0 \\
-255.2 \\
-514.4 \\
-759.2 \\
-871.2 \\
-972.8 \\
-1059.2 \\
-1138.4 \\
-1189.6 \\
-1167.2 \\
-797.6 \\
\end{array}$ & $\begin{array}{l}0.0 \\
-.214 \\
-.587 \\
-1.022 \\
-1.247 \\
-1.473 \\
-1.700 \\
-1.992 \\
-2.332 \\
-2.736 \\
-3.363 \\
\end{array}$ & $\begin{array}{l}0.0 \\
.039 \\
.079 \\
.156 \\
.196 \\
.263 \\
.334 \\
.476 \\
.695 \\
1.038 \\
1.844 \\
\end{array}$ & $\begin{array}{l}0.0 \\
-.011 \\
.025 \\
.016 \\
.014 \\
.036 \\
.001 \\
-.021 \\
-.100 \\
-.090 \\
-.136 \\
\end{array}$ & $\begin{array}{l}0.0 \\
-.299 \\
-.376 \\
-.642 \\
-.759 \\
-.920 \\
-1.023 \\
-1.262 \\
-1.341 \\
-1.146 \\
-.600 \\
\end{array}$ & $\begin{array}{l}0.0 \\
.034 \\
.115 \\
.173 \\
.218 \\
.274 \\
.376 \\
.542 \\
.895 \\
1.238 \\
1.963 \\
\end{array}$ & $\begin{array}{l}0.0 \\
-.035 \\
-.008 \\
.004 \\
-.001 \\
-.004 \\
.016 \\
.004 \\
.073 \\
.283 \\
.742 \\
\end{array}$ \\
\hline
\end{tabular}

Tabelle B11: R1lemversuch R13

\begin{tabular}{|c|c|c|c|c|c|c|c|c|}
\hline \multirow{2}{*}{$\begin{array}{l}\text { Last- } \\
\text { stufe }\end{array}$} & \multirow{2}{*}{$\begin{array}{c}\text { Anfangs - } \\
\text { last } \\
F_{\mathrm{A}} \\
{[\mathrm{kN}]}\end{array}$} & \multirow{2}{*}{$\begin{array}{c}\text { Endlast } \\
F_{E} \\
{[K N]}\end{array}$} & \multicolumn{3}{|c|}{ Verzerrungen Mauerwerk } & \multicolumn{3}{|c|}{ Verzerrungen Stein } \\
\hline & & & $\varepsilon_{x} \cdot 10^{3}$ & $\varepsilon_{y} \cdot 10^{\prime}$ & $\gamma_{x y} \cdot 10^{\prime}$ & $\varepsilon_{x} \cdot 10^{3}$ & $\varepsilon_{y} \cdot 10^{\prime}$ & $\gamma_{x y} \cdot 10^{\prime}$ \\
\hline $\begin{array}{l}1 \\
2 \\
3 \\
4 \\
5 \\
6 \\
7\end{array}$ & $\begin{array}{r}0.0 \\
-201.6 \\
-391.2 \\
-644.8 \\
-900.8 \\
-1020.8 \\
-1084.8 \\
\end{array}$ & $\begin{array}{r}0.0 \\
-204.8 \\
-388.0 \\
-638.4 \\
-890.4 \\
-1010.4 \\
-1072.0 \\
\end{array}$ & $\begin{array}{l}0.0 \\
-.353 \\
-.625 \\
-.933 \\
-1.350 \\
-1.581 \\
-1.667\end{array}$ & $\begin{array}{l}0.0 \\
.021 \\
.067 \\
.121 \\
.189 \\
.231 \\
.538\end{array}$ & $\begin{array}{l}0.0 \\
-.065 \\
-.002 \\
-.001 \\
.042 \\
.044 \\
-.089\end{array}$ & $\begin{array}{r}0.0 \\
-.132 \\
-.313 \\
-.623 \\
-.969 \\
-1.086 \\
-1.142\end{array}$ & $\begin{array}{l}0.0 \\
.061 \\
.089 \\
.171 \\
.284 \\
.412 \\
.696\end{array}$ & $\begin{array}{l}0.0 \\
-.025 \\
-.007 \\
-.012 \\
.059 \\
.082 \\
-.071\end{array}$ \\
\hline
\end{tabular}

Tabelle B12: Rilemversuch R14

\begin{tabular}{|c|c|c|c|c|c|c|c|c|}
\hline \multirow{2}{*}{$\begin{array}{l}\text { Last- } \\
\text { stufe }\end{array}$} & \multirow{2}{*}{$\begin{array}{c}\text { Anf angs- } \\
\text { last } \\
F_{\mathrm{A}} \\
{[\mathrm{kN}]}\end{array}$} & \multirow{2}{*}{$\begin{array}{c}\text { Endlast } \\
\mathrm{FE}^{\mathrm{F}} \\
{[\mathrm{kN}]}\end{array}$} & \multicolumn{3}{|c|}{ Verzerrungen Mauerwerk } & \multicolumn{3}{|c|}{ Verzerrungen steln } \\
\hline & & & $\varepsilon_{x} \cdot 10^{3}$ & $\varepsilon_{y} \cdot 10^{3}$ & $\gamma_{x y} \cdot 10^{3}$ & $\varepsilon_{X} \cdot 10^{\prime}$ & $e_{y} \cdot 10^{2}$ & $\gamma_{x y} \cdot 10^{\prime}$ \\
\hline $\begin{array}{r}1 \\
2 \\
3 \\
4 \\
5 \\
6 \\
7 \\
8 \\
9 \\
10 \\
11 \\
12 \\
13 \\
14\end{array}$ & $\begin{array}{r}0.0 \\
-101.6 \\
-192.8 \\
-288.8 \\
-380.0 \\
-465.6 \\
-544.0 \\
-607.2 \\
-641.6 \\
-676.0 \\
-682.4 \\
-694.4 \\
-690.4 \\
-676.0\end{array}$ & $\begin{array}{r}0.0 \\
-100.0 \\
-186.4 \\
-281.6 \\
-371.2 \\
-453.6 \\
-529.6 \\
-592.0 \\
-626.4 \\
-645.6 \\
-660.8 \\
-672.8 \\
-660.0 \\
-649.6\end{array}$ & $\begin{array}{l}0.0 \\
-.215 \\
-.384 \\
-.544 \\
-.748 \\
-.950 \\
-1.123 \\
-1.397 \\
-1.525 \\
-1.628 \\
-1.820 \\
-1.978 \\
-2.198 \\
-2.433\end{array}$ & $\begin{array}{l}0.0 \\
-.001 \\
.021 \\
.048 \\
.062 \\
.094 \\
.090 \\
.156 \\
.186 \\
.253 \\
.419 \\
.542 \\
.750 \\
.997\end{array}$ & $\begin{array}{l}0.0 \\
.015 \\
.000 \\
.018 \\
.025 \\
.046 \\
-.010 \\
-.044 \\
-.043 \\
-.092 \\
-.111 \\
-.053 \\
-.023 \\
-.030\end{array}$ & $\begin{array}{l}-.259 \\
-.352 \\
-.393 \\
-.449 \\
-.595 \\
-.611 \\
-.760 \\
-.834 \\
-.781 \\
-.894 \\
-.827 \\
-.761 \\
-.690\end{array}$ & $\begin{array}{l}0.0 \\
-.003 \\
.004 \\
.027 \\
.054 \\
.087 \\
.109 \\
.119 \\
.160 \\
.314 \\
.583 \\
.780 \\
.967 \\
1.111\end{array}$ & $\begin{array}{c}0.0 \\
.016 \\
.009 \\
-.015 \\
.002 \\
-.008 \\
.008 \\
-.004 \\
.026 \\
.016 \\
.040 \\
.078 \\
.234 \\
.425\end{array}$ \\
\hline
\end{tabular}

Tabelle B13: Rilemversuch R15 


\begin{tabular}{|c|l|c|c|}
\hline & Hersteller & Produktionsdatum & Bezeichnungen \\
\hline B & Rafz & 9.10 .81 & \\
C & Prungen & 4.8 .83 & \\
D & Fräschels & 31.8 .83 & \\
F & Aarberg & & \\
G & Hochdorf & 23.6 .83 & \\
H & Frick & & \\
I & Frick & 22.123 .12 .83 & \\
I1 & Frick & 27.4 .84 & \\
J & Frick & 25.11 .83 & \\
J1 & Frick & 1.12 .83 & \\
K & Paradies & & 43/83 D \\
L & Hardwald & & OCA 27 KH \\
M & Hunziker, 0lten & & \\
\hline
\end{tabular}




\begin{tabular}{|c|c|c|c|c|c|c|}
\hline \multirow[t]{2}{*}{$\begin{array}{l}\text { Vers.- } \\
\text { serie }\end{array}$} & \multicolumn{5}{|c|}{$\begin{array}{l}\text { Zuordnung der Versuche zu dem } \\
\text { Fugenneigungswinkel a }\end{array}$} & \multirow[t]{2}{*}{$\begin{array}{l}\text { RILEM- } \\
\text { versuche }\end{array}$} \\
\hline & $\alpha=0^{\circ}$ & $22.5^{\circ}$ & $45^{\circ}$ & $67.5^{\circ}$ & $90^{\circ}$ & \\
\hline 2 & K 62 & $\begin{array}{lll}K & 13 \\
K & 14 \\
K & 15\end{array}$ & $\begin{array}{ll}\mathrm{K} & 16 \\
\mathrm{~K} & 17 \\
\mathrm{~K} & 18\end{array}$ & K 63 & K 53 & $\begin{array}{l}R \quad, 2,3 \\
R \quad 4,5,6\end{array}$ \\
\hline 3 & K 19 & K 20 & & & K 54 & R 7 \\
\hline 4 & K 21 & K 22 & K 23 & K 24 & K 55 & R 8 \\
\hline 5 & K 25 & K 26 & K 27 & K 28 & K 56 & \\
\hline 6 & K 29 & K 30 & K 31 & K 32 & K 57 & \\
\hline 7 & K 33 & K 34 & K 35 & K 36 & K 58 & R 10 \\
\hline 8 & K 37 & K 38 & & & K 59 & R 11 \\
\hline 9 & K 39 & K 40 & K 41 & K 42 & & \\
\hline 10 & K 43 & K 44 & & & & \\
\hline 11 & K 45 & K 46 & K 47 & K 48 & K 60 & A 12 \\
\hline 12 & K 49 & K 50 & K 51 & K 52 & K 61 & R 13 \\
\hline
\end{tabular}
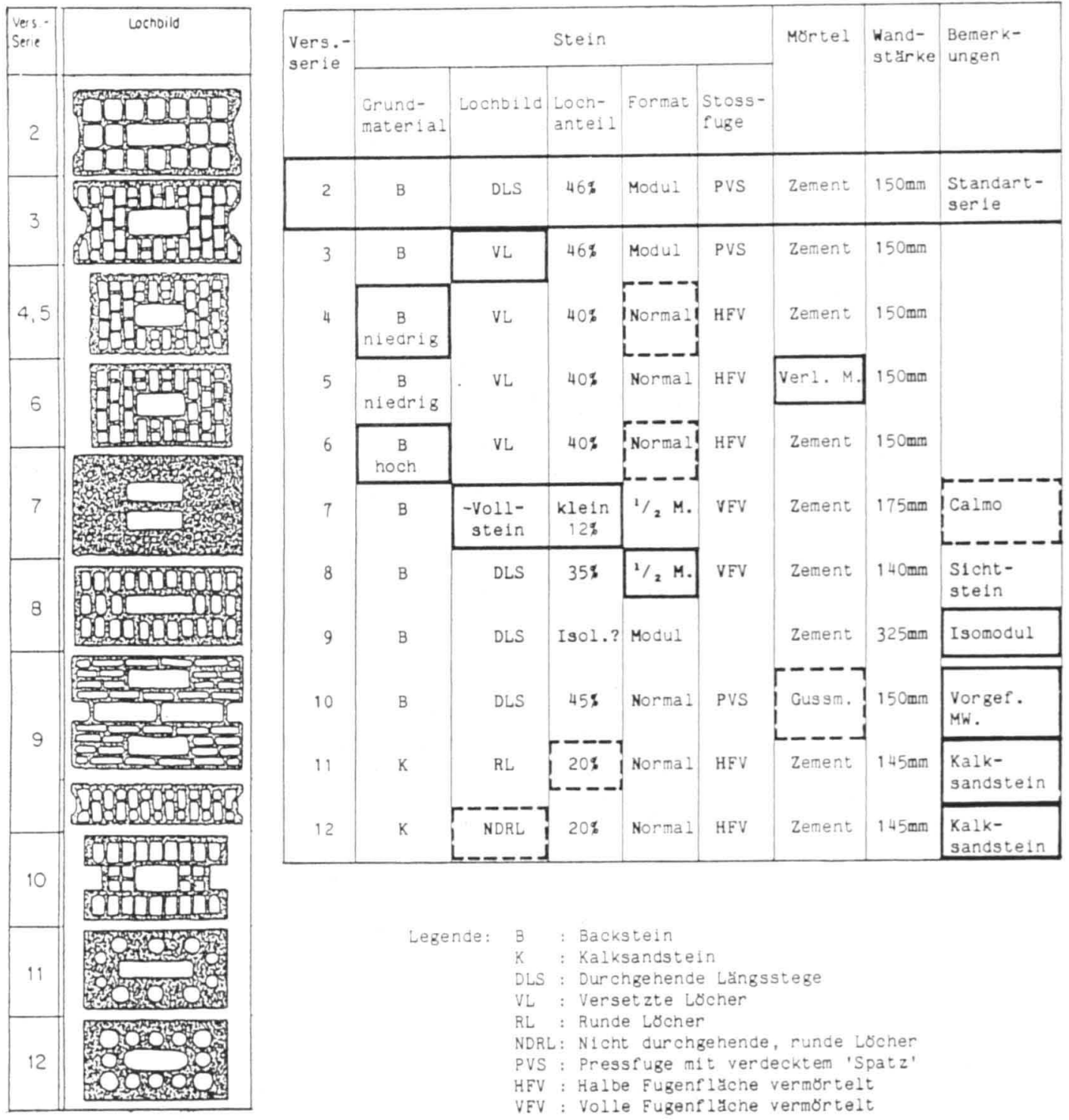